\title{
40. EARLY TO MIDDLE MIOCENE RADIOLARIAN STRATIGRAPHY OF THE KERGUELEN PLATEAU, LEG 1201
}

\author{
Andrea Abelmann ${ }^{2}$
}

\begin{abstract}
Early to middle Miocene radiolarian assemblages were examined at three sites $(747,748$, and 751$)$ that were cored during Ocean Drilling Program Leg 120 south of the present polar frontal zone on the Kerguelen Plateau (Indian sector of the Southern Ocean). The radiolarian biostratigraphic study relies on a radiolarian zonation recently developed on Leg 113 materials in the Atlantic sector of the Southern Ocean, which is correlated with the geomagnetic time scale. New radiolarian biostratigraphic data also considering the established geomagnetic polarity record were used to improve and emend the age calibration of some lower Miocene radiolarian zones and a redefined middle Miocene radiolarian zonation is proposed. Based on these results, a revised age assignment of the lower Miocene sections drilled at Leg 113 Sites 689 and 690 is proposed.
\end{abstract}

\section{INTRODUCTION}

Ocean Drilling Program (ODP) Leg 120, the last of a series of four legs to the Southern Ocean (Legs 113, 114, 119, and 120), recovered Cenozoic sections at five sites in the Indian sector of the Southern Ocean. These sites completed a transect of 16 drill sites between the East Antarctic continental shelf (Prydz Bay) and the northern area of the Kerguelen Plateau, started during Leg 119. The main scientific objectives of Legs 119 and 120 were to document the Cenozoic paleoceanographic and paleoclimatic evolution in the Indian sector of the Southern Ocean and to reconstruct the glacial history of East Antarctica. One additional objective was the further development of an integrated siliceous and calcareous biostratigraphy calibrated to the geomagnetic time scale, and the testing or revision of the biostratigraphic zonations that have been previously established for the southern high latitudes based on sediment sequences recovered in the Atlantic and Pacific sectors of the Southern Ocean (e.g., Hayes and Frakes, 1975; Weaver et al., 1976; Basov et al., 1983; Barker, Kennett, et al., 1988; Gersonde et al., 1990; Barron et al., 1991). The establishment of an integrated biomagnetic and geomagnetic stratigraphy for the Southern Ocean would allow its incorporation into a global stratigraphic framework and thus would be the base for an accurate global intercorrelation of paleoceanographic events and paleoclimatic changes.

This chapter focuses on the radiolarian biostratigraphy of lower to middle Miocene pelagic sediment sequences that were recovered in Holes $747 \mathrm{~A}, 748 \mathrm{~A}$, and $751 \mathrm{~A}$, drilled on the Kerguelen Plateau, south of the present Polar Frontal Zone (Fig. 1). The Paleogene radiolarians were studied by Takemura (this volume), and the upper Miocene to Holocene radiolarians by Lazarus (this volume). The Miocene sections of the three sites investigated were continuously recovered using advanced hydraulic piston coring (APC). The sediments are composed of biosiliceous and calcareous microfossils (Schlich, Wise, et al., 1989), containing well-preserved radiolarian assemblages in the intervals studied from Holes $748 \mathrm{~A}$ and $751 \mathrm{~A}$, whereas in Hole $747 \mathrm{~A}$ the preservation of the

\footnotetext{
${ }^{1}$ Wise, S. W., Jr., Schlich, R., et al., 1992. Proc. ODP, Sci. Results, 120: College Station, TX (Ocean Drilling Program).

2 Alfred Wegener Institute for Polar and Marine Research, D-2850 Bremerhaven, Columbusstrasse, Federal Republic of Germany.
}

radiolarians is generally poor (Table 1). A geomagnetic polarity scheme was developed for the sections studied by Heider (this volume) for Site 747 and 751 and by Inokuchi (this volume) for Site 748 . However, the occurrence of disconformities and the presence of significant gaps in the geomagnetic record because of intervals with uninterpretable data disturb the geomagnetic polarity pattern and complicate its stratigraphic interpretation in some intervals.

Also presented in this chapter are age assignments for each of the Leg 120 sediment sequences studied as well as for the disconformities based on radiolarian biostratigraphic zonations recently developed during drilling on Leg 113 Sites 689 and 690 (Atlantic sector of the Southern Ocean, Maud Rise), which were correlated with the geomagnetic time scale of Abelmann (1990) and the geomagnetic polarity patterns reported in the individual Leg 120 site chapters (Schlich, Wise, et al., 1989). Additional stratigraphic support was obtained by comparing the diatom stratigraphic data from Leg 113 (Gersonde and Burckle, 1990), Leg 119 (Baldauf and Barron, 1991), and Leg 120 (Harwood and Majurama, this volume). The new radiolarian biostratigraphic data that resulted from this study were used to improve and revise the age calibration of the lower Miocene radiolarian zones established by Abelmann (1990) and to redefine the middle Miocene radiolarian zonation.

\section{METHODS}

Samples were taken aboard JOIDES Resolution during Leg 120. The samples were washed through a $40-\mu \mathrm{m}$ mesh sieve, and randomly distributed slides were prepared according to the methods described by Abelmann (1989) and Abelmann et al. (unpubl. data). Light microscopic investigations were made with a Leitz Orthoplan foto-microscope with apochromatic optics. Species identification was done routinely at $160 \times$ or $250 \times$. The five semiquantitative abundance categories used to estimate radiolarian numbers were defined as $\mathrm{A}=$ abundant $(>50 \%$ of the total assemblage $), \mathrm{C}=$ common $(15 \%-50 \%), \mathrm{F}=$ few $(3 \%-15 \%), \mathrm{R}=$ rare $(1 \%-3 \%)$, and $\mathrm{T}=$ trace $(<1 \%)$. The preservation of radiolarian assemblages is classified as $\mathrm{P}=$ poor, $\mathrm{M}=$ moderate, and $\mathrm{G}=$ good, according to the degree of dissolution and breakage of the radiolarian shells (see Tables 2 through 4 ).

\section{RADIOLARIAN ZONATION}

The first radiolarian zonation for Antarctic sediments was established by Hays and Opdyke (1967) for the Pliocene and 


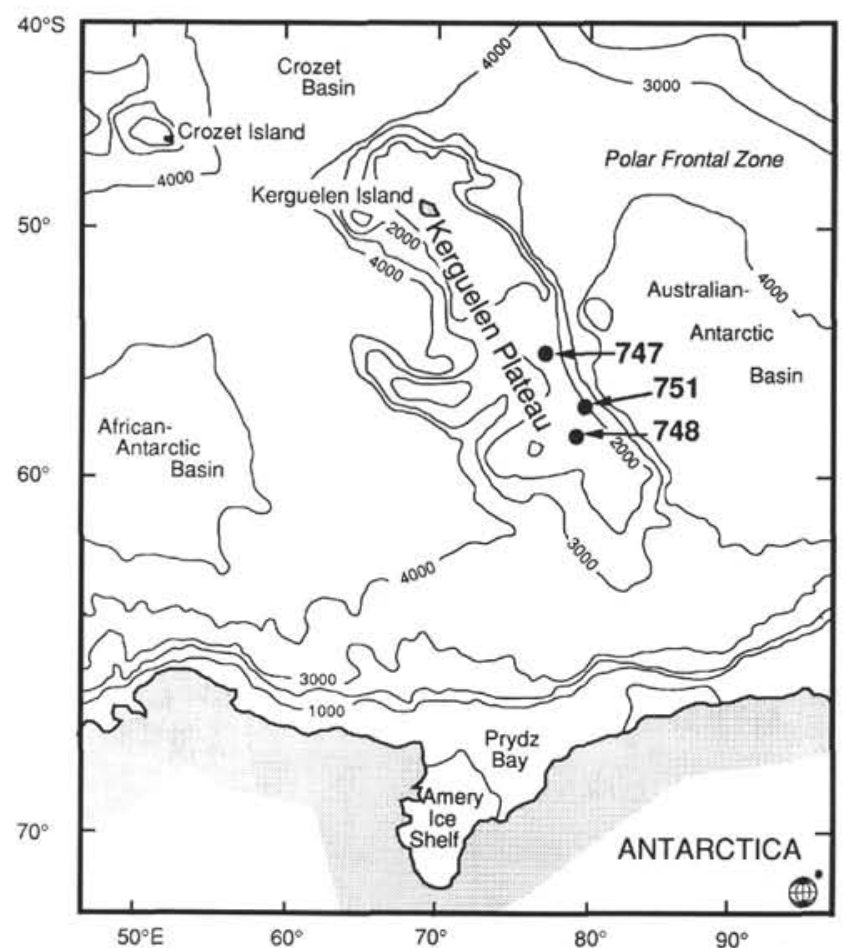

Figure 1. Location of Sites 747,748 , and 751 on the Kerguelen Plateau. Contour intervals are given in meters.

Pleistocene time interval using piston cores recovered in the Pacific sector of the Antarctic Ocean. Based on rotarydrilled material during Deep Sea Drilling Program (DSDP) Leg 28 in the Indian and Pacific sectors of the Southern Ocean, Chen (1975) developed a detailed zonation for the Miocene that was accepted by many subsequent workers. An independent zonation was established by Petrushevskaya (1975) on DSDP Leg 29 material (Pacific sector) for the Neogene and Paleogene. Weaver $(1976,1983)$ used the radiolarian zonations of Hays and Opdyke (1967) and Chen (1975) for the age assignment of Subantarctic and Antarctic sediments recovered on DSDP Legs 35 and 71 (Bellingshausen Sea, Falkland Plateau) with some modifications.
Although the Pliocene and Pleistocene radiolarian zonation for Antarctic sediments was already correlated to the geomagnetic time scale by Hays and Opdyke (1967), and subsequently by Keany (1979) and Weaver (1983), the Miocene and Paleogene radiolarian zonations for the southern high latitudes were not calibrated because no geomagnetic measurements were available for the DSDP cores studied. It was only during ODP Legs $113,114,119$, and 120 that well-preserved and continuously recovered sediment cores were gathered in the Southern Ocean, which allowed the establishment of geomagnetic polarity records for Miocene and Paleogene sediment sequences. Based on sequences recovered during Leg 113 at Sites 689 and 690 on the Maud Rise (Weddell Sea), which represent the first Antarctic sites in which the APC drilling technique was used successfully, Abelmann (1990) proposed a revised radiolarian zonation for the late Oligocene to middle Miocene time interval, which is correlated to the geomagnetic polarity record established by Spieß (1990). The correlation of upper Miocene and Pliocene radiolarian zones, which were reported by Lazarus (1990) at the same sites, is summarized in Gersonde et al. (1990).

Abelmann (1990) proposed five new radiolarian zones and modified five zones previously defined by Chen (1975) for the late Oligocene to middle Miocene time interval (Fig. 2). As far as possible, the first appearance datum (FAD) of species was used to define the zones. Abelmann's stratigraphic zonation and its calibration to the geomagnetic time scale is used in this chapter. Additional data gathered during the present investigation allowed the improvement of the zonation established on Leg 113 materials. In particular, the stratigraphic age assignment of the lower Miocene zones and species ranges proposed by Abelmann (1990) could be emended (Fig. 2). However, disconformities and gaps in the geomagnetic record complicate the accurate chronostratigraphic interpretation in some intervals and the age ranges of some of the radiolarian zones must still be tentative (Fig. 3 ).

To estimate the ages of the zonal boundaries, the subbottom depths (in meters below seafloor [mbsf]) were first determined for all geomagnetic polarity boundaries and radiolarian zonal boundaries (see Figs. 4-6). The sub-bottom depths of radiolarian zonal boundaries were calculated as the midpoint between studied samples that bracketed the zonal boundaries. Zonal boundary ages were then calculated by assuming constant sediment accumulation rates between adjacent chron or subchron boundaries. The age determinations of hiatuses were done in the same way.

Table 1. Radiolarian datum levels of stratigraphic marker species for the early to middle Miocene time interval, with age assignment and depth definition (mbsf).

\begin{tabular}{|c|c|c|c|c|c|}
\hline Event & Species name & $\begin{array}{c}\text { Known age } \\
\text { (Ma) }\end{array}$ & $\begin{array}{c}\text { Hole } 747 \mathrm{~A} \\
\text { occurrence level } \\
\text { (mbsf) }\end{array}$ & $\begin{array}{c}\text { Hole 748B } \\
\text { occurrence level } \\
\text { (mbsf) }\end{array}$ & $\begin{array}{c}\text { Hole 751A } \\
\text { occurrence level } \\
\text { (mbsf) }\end{array}$ \\
\hline $\mathrm{T}$ & Stylosphaera radiosa & $\sim ? 22.6-? 22.2$ & $120-118.8$ & ${ }^{*} 60.6-59.1$ & \\
\hline B & Cyrtocapsella tetrapera & $\sim ? 22.6-? 22.2$ & $120-118.8$ & $* 60.6-59.1$ & ${ }^{*} 166.2$ \\
\hline B & Cycladophora antiqua & $\sim ? 22.6-? 22.2$ & & $* 60.6-59.1$ & ${ }^{*} 166.2$ \\
\hline B & Cyrtocapsella longithorax & $\sim ? 21.9-? 20.2$ & $* 85.0-85.5$ & $* 57.6-59.1$ & ${ }^{*} 166.2$ \\
\hline B & Cycladophora golli regipileus & $19.5-19.3$ & $* 93.0-94.5$ & $* 57.6-59.1$ & $159.2-162.2$ \\
\hline B & Gondwanaria deflandrei & $\sim 19.5-19.3$ & & $57.6-59.1$ & $157.7-162.2$ \\
\hline B & Eucyrtidium punctatum & $\sim 17.3$ & $* 94.5-95.0$ & $* 38.7-40.1$ & $137.7-138.7$ \\
\hline B & Cycladophora humerus & 14.2 & $75.5-77.5$ & $* 38.7-35.1$ & $112.5-113.2$ \\
\hline B & Lychnocanoma sp. B & 14.2 & $75.5-77.5$ & & $112.5-113.2$ \\
\hline B & Antarctissa deflandrei & $\sim 14$ & $* 58.5-60.0$ & & $* 109.2-109.5$ \\
\hline B & Actinomma golownini & 13.1 & $* 65.7-66$ & $* 38.7-35.3$ & ${ }^{*} 109.2-109.5$ \\
\hline B & Dendrospyris megalocephalis & $\sim 12.4-12.5$ & & & $* 109.2-109.5$ \\
\hline B & Cycladophora spongothorax & $\sim 11.8$ & $* 55.0-56.5$ & $* 38.7-35.3$ & $99.7-101$ \\
\hline
\end{tabular}

Note: For first occurrence levels, the first appearance datum may be older.

*Data not useable. 


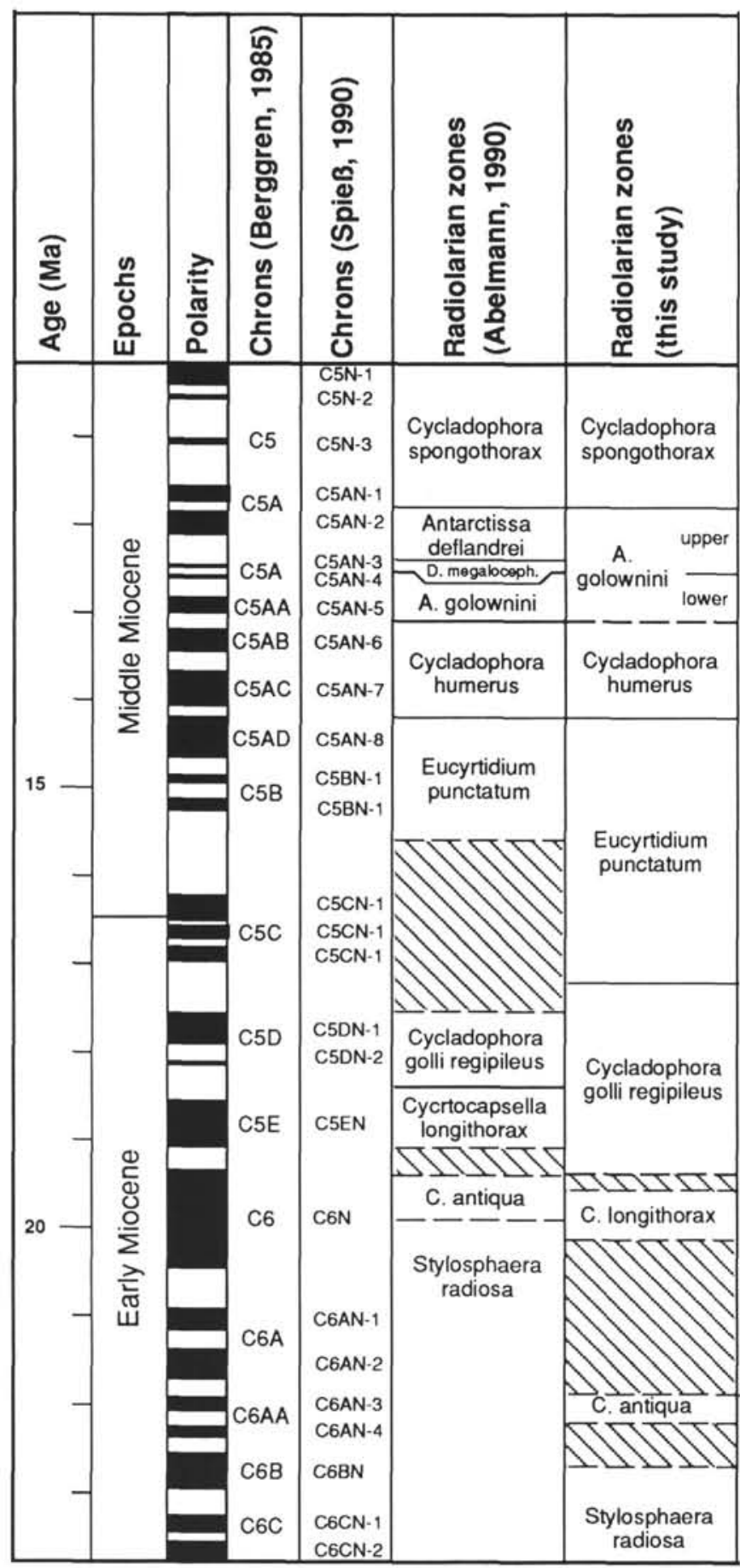

Figure 2. Comparison of early to middle Miocene radiolarian zonations proposed by Abelmann (1990) on Leg 113 materials and in this study, and their correlation to the geomagnetic time scale of Berggren et al. (1985). Shaded zone intervals indicate the range of uncertainty of the zonal boundary age.

To designate paleomagnetic events, the nomenclature proposed by Tauxe et al. (1984) was used. The absolute age assignment is according to the geomagnetic polarity time scale of Berggren et al. (1985).

\section{Actinomma golownini Zone ( 13.1 to $\sim 11.8 \mathrm{Ma})$}

Top. First appearance of Cycladophora spongothorax.

Base. First appearance of Actinomma golownini.

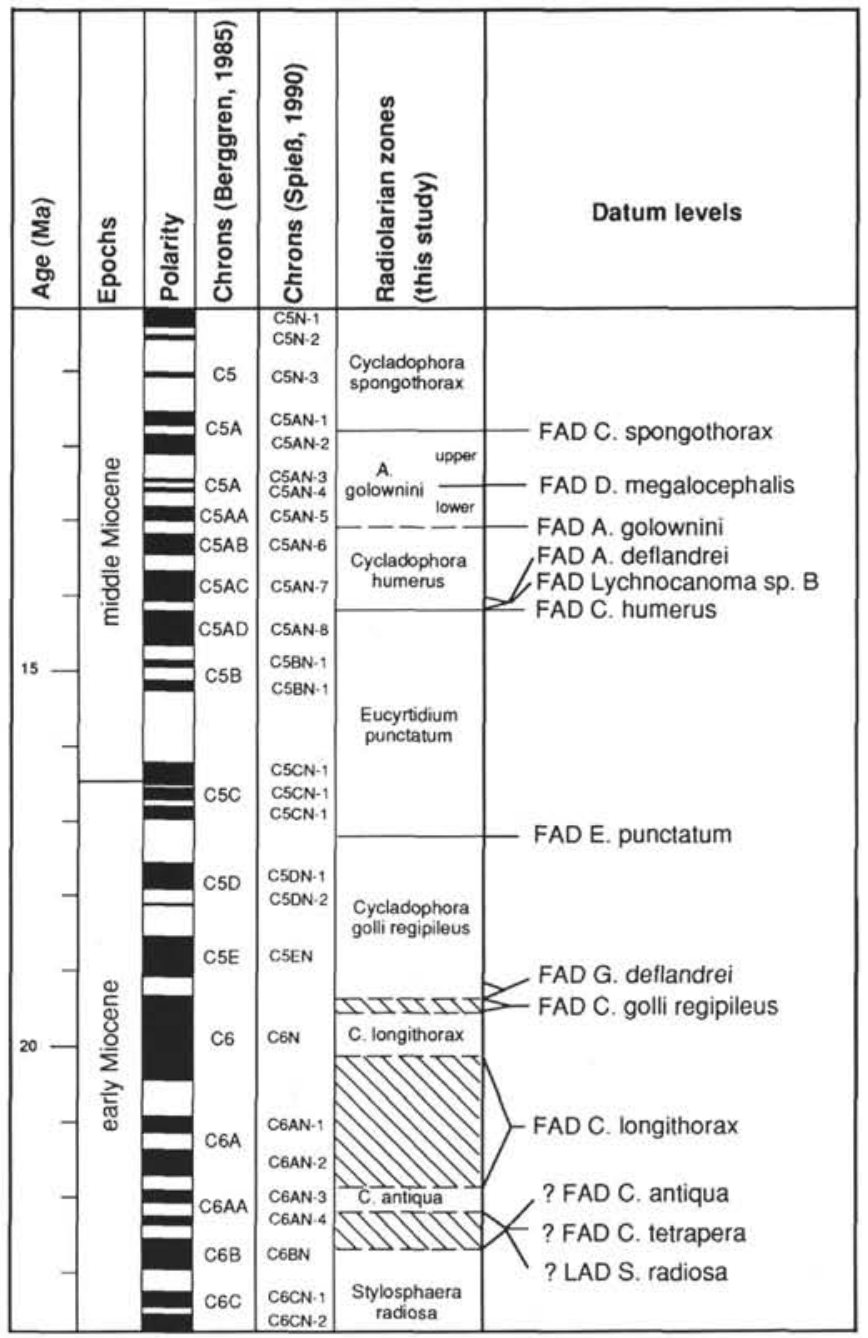

Figure 3. Proposed radiolarian zonation for early to middle Miocene Antarctic sediments and datum levels of stratigraphic marker species, and their correlation to the geomagnetic time scale of Berggren et al. (1985). Shaded zone intervals indicate the range of uncertainty of the zonal boundary ages and species datums.

Author. Chen (1975), as Actinomma tanyacantha Zone, renamed herein.

Remarks. The zone is divided into lower and upper subzones defined by the FAD of Dendrospyris megalocephalis, which was placed at $\sim 12.45 \mathrm{Ma}$.

Paleomagnetic correlation. The top of the zone is placed between the uppermost normal events in C5A, the base between Subchrons C5AA and C5AB. The boundary between the upper and lower $A$. golownini zones is placed between the two lower normal events of Subchron C5A (Fig. 3).

Discussion. This zone was originally described by Chen (1975) as the Actinomma tanyacantha Zone. According to the International Rules of Zoological Nomenclature, Chen's species name A. tanyacantha is not valid and Petrushevskaya's name A. golownini has priority because her paper published in the DSDP Leg 29 volume (January 1975) was delivered before Chen's publication in the DSDP Leg 28 volume (July 1975). Thus, the zonal name has to be changed to the A. golownini Zone.

Abelmann (1990) modified this zone at its top by introducing two new zones, the Dendrospyris megalocephalis and the Antarctissa deflandrei zones, which correspond to the upper portion of the $A$. golownini Zone, between 12.45 and $11.8 \mathrm{Ma}$ (Fig. 2). However, during this study, the practicability of the middle Miocene $D$. megalocephalis Zone proposed by Abelmann (1990) was found to be problematic. The 
zone is defined by the total range of $D$. megalocephalis, which only spans the short time interval between approximately 12.45 and 12.3 $\mathrm{Ma}$. The last appearance datum (LAD) of D. megalocephalis also defines the base of the younger A. deflandrei Zone of Abelmann. This leads to the problem that, in case D. megalocephalis is omitted because of a hiatus or other reasons, the $A$. deflandrei Zone cannot be delineated. Because other stratigraphic marker species could not be defined for the discussed time interval, the $D$. megalocephalis and the A. deflandrei zones of Abelmann (1990) are reincorporated into the Actinomma golownini Zone. However, using the FAD of D. megalocephalis, the $A$. golownini Zone can be subdivided into an upper and a lower subzone.

\section{Cycladophora humerus Zone (14.2 to $\sim 13.1 \mathrm{Ma})$}

Top. First appearance of Actinomma golownini.

Base. First appearance of Cycladophora humerus.

Author. Abelmann (1990).

Paleomagnetic correlation. The top of the zone is placed between Subchrons C5AA and C5AB, and the base at the top of Subchron C5AD (Fig. 3).

Discussion. An additional stratigraphic mark near the base of this zone is the FAD of Lychnocanoma sp. B. Near the zonal base, the probable evolutionary transition from Antarctissa robusta to A. deflandrei is completed (Abelmann, 1990).

\section{Eucyrtidium punctatum Zone ( 17.3 to 14.2 Ma)}

Top. First appearance of Cycladophora humerus.

Base. First appearance of Eucyrtidium punctatum.

Author. Chen (1975), top modified by Abelmann (1990).

Paleomagnetic correlation. The top of the zone is placed at the top of Subchron C5AD, and the base occurs in the reversed interval between Subchron C5C and the top of Subchron C5D (Fig. 3).

Discussion. Based on Leg 113 materials (Abelmann, 1990) the base of the E. punctatum Zone was placed somewhere between Subchron C5B and the top of Subchron C5D, that is, in a range between 15.6 and 17.6 Ma (Fig. 2). Because the lower portion of the zone is omitted as a result of a disconformity in Leg 113 Holes 689B and 690B, a more precise age determination of the zonal base was not feasible. Based on the integrated geomagnetic (Heider, this volume) and biostratigraphic results gathered at Hole $751 \mathrm{~A}$, the age determination of the zonal base can be improved. The base can be placed somewhere in the reversed interval between Subchron C5C and the top of Subchron C5D at approximately $17.3 \mathrm{Ma}$ (Fig. 6).

In the original description of Chen (1975), the top of the $E$. punctatum Zone was defined by the FAD of Spongomelissa dilli. Because $S$. dilli was not found in Leg 113 Holes 689B and 690B, Abelmann (1990) modified Chen's original zonal definition. In this study, $S$. dilli was encountered in Hole $747 \mathrm{~A}$ in a short-ranging interval near the top of the $C$. humerus Zone and in Hole 751A in the upper portion of the A. golownini Zone (Figs. 4 and 6; Tables 2 and 4).

\section{Cycladophora golli regipileus Zone$$
(\sim 19.5 / 19.3 \text { to } \sim 17.3 \mathrm{Ma})
$$

Top. First appearance of Eucyrtidium punctatum.

Base. First appearance of Cycladophora golli regipileus.

Author. Chen (1975), modified by Abelmann (1990).

Paleomagnetic correlation. The top of the zone is placed in the reversed interval between Subchrons C5C and the top of Subchron C5D, and the base in the upper portion of Chron C6 (Fig. 3).

Discussion. Abelmann (1990) placed the base of this zone near the top of Subchron C5E (18.4 Ma), based on an integrated geomagnetic and biostratigraphic age interpretation (Fig. 2). However, in the Leg 113 sections investigated, the lower Miocene and lower middle Miocene sequences are disturbed by a series of disconformities that complicate the stratigraphic age assignment (Gersonde et al., 1990).

In this study, Abelmann's age determination can be revised based on results gathered in Holes 748B and 751A. In Hole 748B the lower boundary of the $C$. golli regipileus Zone is marked by a hiatus, and in Hole $751 \mathrm{~A}$ the geomagnetic polarity pattern is not interpretable for this time interval. This impedes a precise age determination, however; compared with the results obtained on the Leg 113 materials, the Leg 120 data indicate an older age for the zonal base. In Hole 748B it can be placed somewhere in the upper portion of C6 at around 19.5-19.3
Ma (Fig. 5). In Hole 751A the base of the $C$. golli regipileus Zone is situated below a normal geomagnetic polarity interval interpreted to represent Subchron C5E (Fig. 6).

An additional stratigraphic marker in the lower portion of this zone is the FAD of Gondwanaria deflandrei.

\section{Cyrtocapsella longithorax Zone $(? 21.9 / 20.2$ to $\sim 19.5 / 19.3 \mathrm{Ma})$}

Top. First appearance of Cycladophora golli regipileus.

Base. First appearance of Cyrtocapsella longithorax.

Author. Abelmann (1990).

Paleomagnetic correlation. The top of the zone can be placed near the top of C6, and the zonal base is tentatively placed somewhere between the lower portion of Chron C6 and the top of Subchron C6AA (Fig. 3).

Discussion. Abelmann (1990) could not determine the accurate stratigraphic position of the zonal base because in the Leg 113 holes investigated the lower portion of this zone is omitted by a disconformity. The top of the zone was placed below the top of Subchron C5E, and the base between Subchrons C5E and C6, resulting in a zonal age range of 19.4 to $19.1 \mathrm{Ma}$. In this study sediments that can be assigned to the $C$. longithorax Zone only occur in the basal portion of Hole $751 \mathrm{~A}$, an interval that lacks geomagnetic data (Fig. 6). In Hole 748A, the $C$. longithorax Zone is omitted by a disconformity (Fig. 5) and in Hole $747 \mathrm{~A}$ the critical sediment interval cannot be zoned because of poorly preserved radiolarian assemblages and the lack of zonal marker species (Fig. 4). However, the data obtained in this study indicate that the age range of the $C$. longithorax Zone must be older compared with the range given by Abelmann (1990). Considering the stratigraphic interpretation of Hole $748 \mathrm{~B}$, the top of the $C$. longithorax Zone can tentatively be placed near the top of Chron C6, and the zonal base is provisionally placed somewhere between the lower portion of Chron C6 and the top of Subchron C6AA.

\section{Cycladophora antiqua Zone (?22.6/22.2 to $\mathbf{2 1 . 9 / 2 0 . 2} \mathrm{Ma})$}

Top. First appearance of Cyrtocapsella longithorax.

Base. First appearance of Cycladophora antiqua.

Author. Abelmann (1990).

Paleomagnetic correlation. Tentatively, the top of the zone is placed between the lower portion of $\mathrm{C} 6$ and the top of Subchron C6AA. The base is preliminarily placed into an interval ranging from the top of Subchron C6B to the reversed interval between the normal events of Subchron C6AA.

Discussion. Occurrences of disconformities in the lowermost Miocene cause the age assignment of this zone to be provisional. Abelmann (1990) tentatively placed the top of the zone between Subchron C5E and Chron C6, and the base in the middle portion of Chron C6. In this study, the C. antiqua Zone is correlated with an older time interval according to integrated bio- and geomagnetostratigraphic results (Fig. 2).

Additional stratigraphic marks in the lower portion of the zone are the LAD of Stylosphaera radiosa and the FAD of Cyrtocapsella tetrapera, according to a comparison of data obtained at Holes $747 \mathrm{~A}$ and 748B. Riedel and Sanfilippo (1978) used the FAD of Cyrtocapsella tetrapera to define the base of the $C$. tetrapera Zone in the low-latitude Miocene (equatorial Pacific). The zonal range can be correlated with an interval between the base of Subchron C6AA (around $22.6 \mathrm{Ma}$ ) and the uppermost portion of Subchron C6A, according to Berggren et al. (1985). A slightly younger age determination (22.2 Ma) of the FAD of $C$. tetrapera was given by Barron et al. (1985) based on DSDP Leg 85 investigations. Assuming a synchronous FAD for the cosmopolitan $C$. tetrapera in low latitudes and in the Southern Ocean sector investigated in this study, an age assignment for the base of the $C$. antiqua Zone estimated around 22 . 6 to $22.2 \mathrm{Ma}$ is supported.

Chen (1975) also recognized the stratigraphic usefulness of the FAD of $C$. tetrapera and proposed a $C$. tetrapera Zone for the southern high latitudes at the base of the lower Miocene. However, the data of Abelmann (1990) indicate that during the early portion of the early Miocene $C$. tetrapera was very probably not distributed throughout the entire Southern Ocean area because the species was not encountered in lower Miocene sediments in Holes 689B and 690B 


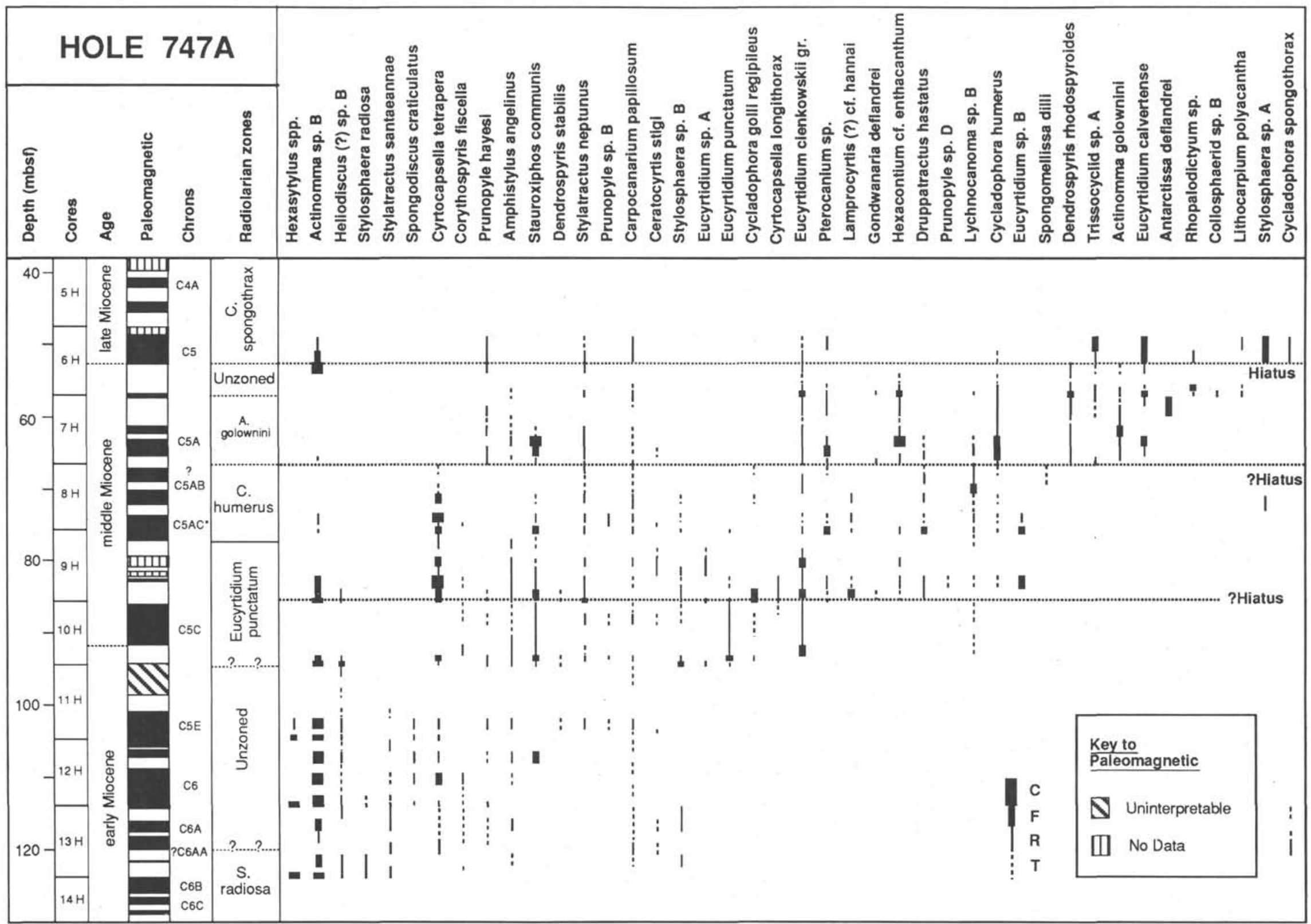

Figure 4. Ranges of selected radiolarian species and radiolarian zonation for early to middle Miocene sediments in Hole 747A, correlated with the geomagnetic polarity pattern established by Heider (this volume). Geomagnetic chron designations were taken from Schlich, Wise, et al. (1989, "Site 747" chapter), except those marked by asterisks. Asterisk-marked chron designations indicate revisions based on the radiolarian biostratigraphic age determination. Hiatuses are indicated by dashed lines. $\mathrm{C}=\mathrm{common}, \mathrm{F}=$ few, $\mathrm{R}=$ rare, and $\mathrm{T}=$ trace. 


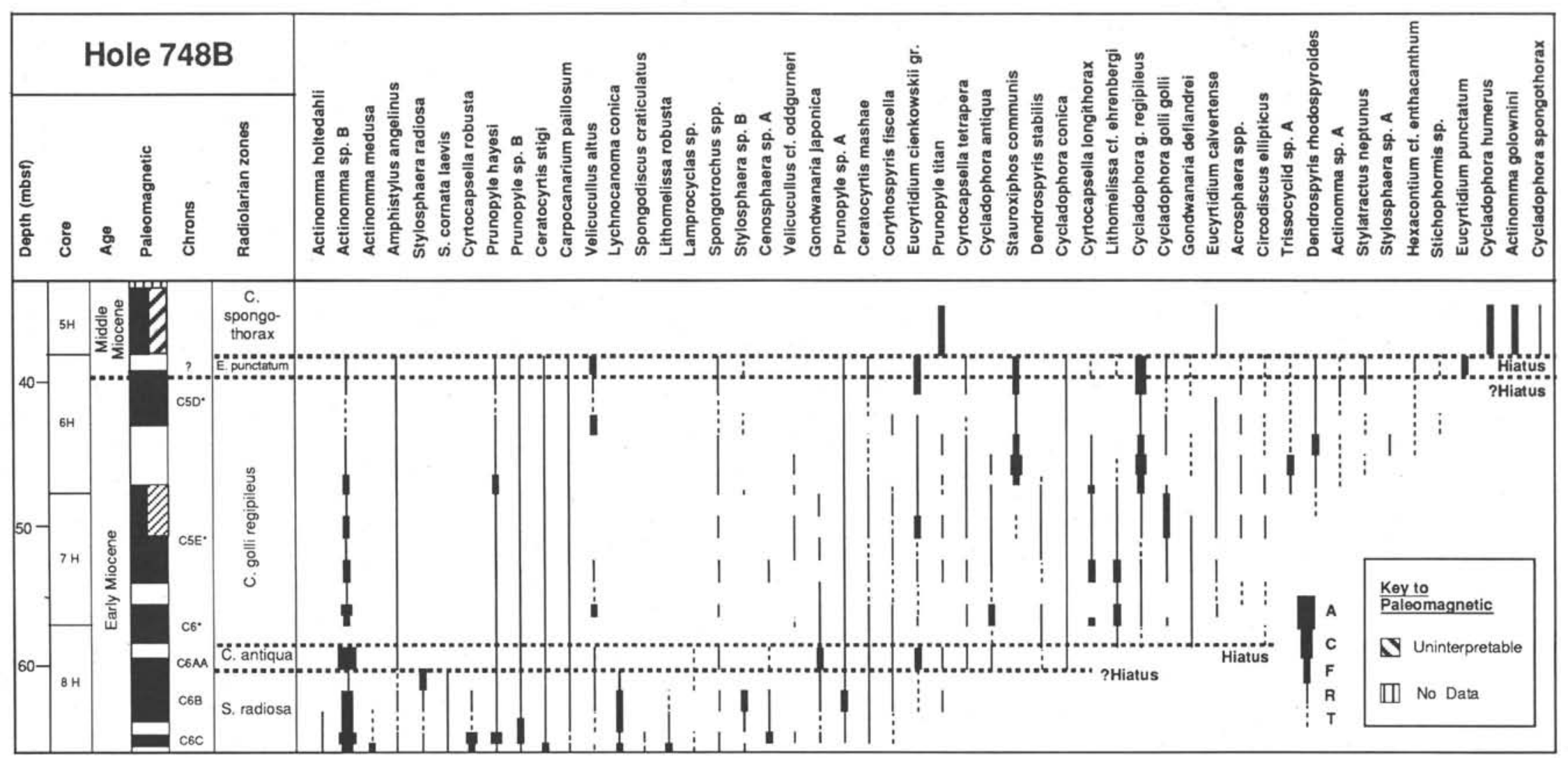

Figure 5. Ranges of selected radiolarian species and radiolarian zonation for early to middle Miocene sediments in Hole $748 \mathrm{~B}$, correlated with the geomagnetic polarity pattern established by Inokuchi (this volume). Geomagnetic chron designations were taken from Schlich, Wise, et al. (1989, "Site 748" chapter), except those marked by asterisks. Asterisk-marked chron designations indicate revisions based on the radiolarian biostratigraphic age determination. Hiatuses are indicated by dashed lines. $\mathrm{A}=\mathrm{abundant}, \mathrm{C}=\mathrm{common}$, $\mathrm{F}=$ few, $\mathrm{R}=$ rare, and $\mathrm{T}=$ trace. 


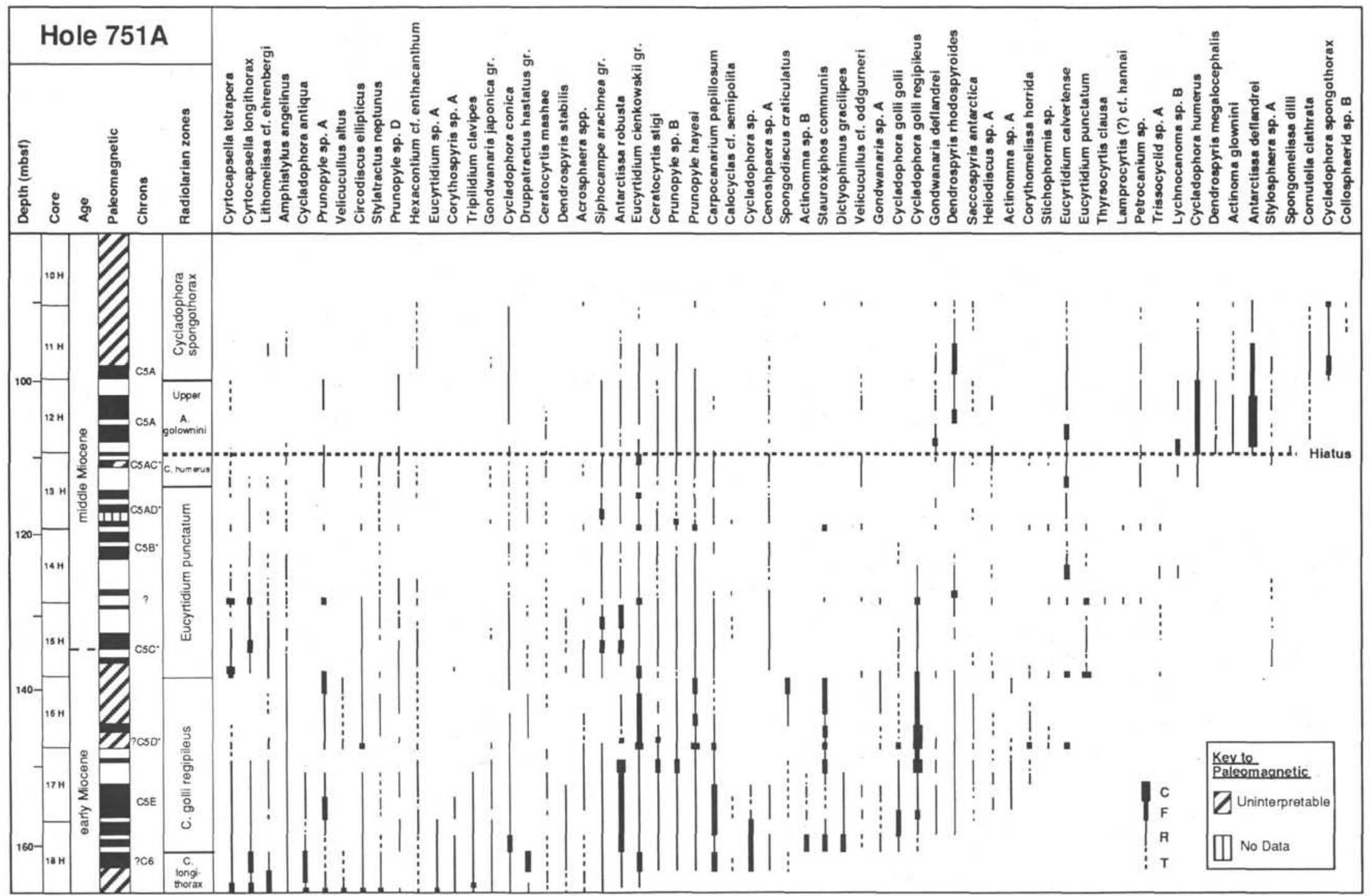

Figure 6. Ranges of selected radiolarian species and radiolarian zonation for early to middle Miocene sediments in Hole $751 \mathrm{~A}$, correlated with the geomagnetic polarity pattern established by Heider (this volume). Geomagnetic chron designations were taken from Schlich, Wise, et al. (1989, "Site 751" chapter), except those marked by asterisks. Asterisk-marked chron designations indicate revisions based on the radiolarian biostratigraphic age determination. Hiatuses are indicated by dashed lines. $\mathrm{C}=\mathrm{common}, \mathrm{F}=$ few, $\mathrm{R}=$ rare, and $\mathrm{T}=$ trace. 
(Maud Rise), which were attributed to the $C$. antiqua and C. longithorax zones. In these holes the first occurrence (FO) of $C$. tetrapera was marked in an interval placed into the Cycladophora golli regipileus Zone, which is correlated to the later portion of the early Miocene. Because emphasis is placed on the applicability of radiolarian zones throughout the entire Southern Ocean, the $C$. tetrapera Zone of Chen (1975) was not adopted for the establishment of a revised radiolarian zonation. However, the usefulness of the FAD of $C$. tetrapera as an additional stratigraphic marker in the early Miocene of the Indian, and probably Pacific, sector of the Southern Ocean is recognized.

Harwood and Maruyama (this volume) placed the FAD of the diatom species Thalassiosira fraga, which occurs near the base of the C. antiqua Zone to a younger time interval between 21.2 and $20.8 \mathrm{Ma}$. The FAD of $T$. fraga was dated in the low- and mid-latitude Pacific between 19.9 and 19.6 Ma (Barron, 1985). Baldauf and Barron (1991) placed the FAD of $T$. fraga between 21 and $20.2 \mathrm{Ma}$, younger than the age termination of Harwood and Maruyama (this volume). Because $C$. tetrapera and $T$. fraga occur in the same core depth, it can be suggested that one species must be diachronous. However, up to now its not yet clear if the base of the $C$. antiqua Zone can be placed at around 22.6 to $22.2 \mathrm{Ma}$ or to an younger time interval between approximately 20.2 and $21.2 \mathrm{Ma}$.

\section{Stylosphaera radiosa Zone (?27.2 to ?22.6/22.2 Ma)}

Top. First appearance of Cycladophora antiqua.

Base. First appearance of Stylosphaera radiosa.

Author. Abelmann (1990).

Paleomagnetic correlation. Tentatively, the top is placed into an interval ranging from the top of $\mathrm{C} 6 \mathrm{~B}$ to the reversed interval between the normal events of C6AA. The base of the zone was not recovered in the sections studied. Abelmann (1990) tentatively placed the base in the middle part of $\mathrm{C} 8$, thus into the uppermost Oligocene (Fig. 2).

Discussion. Abelmann (1990) placed the top of this zone in the middle portion of C6. In this study, the upper zonal boundary is emended to an older age (cf. discussion of $C$. antiqua Zone).

An additional stratigraphic marker is the LAD of Lithomelissa robusta situated in the upper portion of the $S$. radiosa Zone.

\section{RADIOLARIANS AT EACH SITE}

\section{Site $\mathbf{7 4 7}$}

Site 747 is situated in the transition zone between the northern and southern area of the Kerguelen Plateau, approximately $500 \mathrm{~km}$ south of the present Polar Front $\left(54^{\circ} 48.68^{\prime} \mathrm{S}\right.$, $76^{\circ} 47.64^{\prime} \mathrm{E}$ ), at a water depth of $1697 \mathrm{~m}$ (Fig. 1). Its location is the most northerly of the three Kerguelen sites investigated in this chapter. At Site 747, three holes were drilled and a 295-m-thick sequence of Santonian through Pleistocene sediments and $54 \mathrm{~m}$ of the underlying basalt were cored.

Radiolarians in this report were studied from the lower Miocene to the uppermost middle Miocene sediments recovered in Hole 747A between Section 120-747A-13H-CC and Sample 120-747A-6H-2, 45-47 cm, were investigated. Species ranges and the abundance pattern of the studied radiolarians are shown in Table 2 and Figure 4.

The abundance pattern of radiolarian shells is characterized by distinct changes throughout the interval examined in Hole 747A. The lowest numbers of radiolarians were found in the early Miocene between Samples 120-747A-11H-5, 45-47 $\mathrm{cm}$, and $-11 \mathrm{H}-1,45-47 \mathrm{~cm}$, and in two intervals in the middle Miocene between Samples 120-747A-9H-5, 45-47 cm, and $-9 \mathrm{H}-2,45-47 \mathrm{~cm}$, and Samples $120-747 \mathrm{~A}-7 \mathrm{H}-3,45-47 \mathrm{~cm}$, and $-6 \mathrm{H}-5,45-47 \mathrm{~cm}$. All other intervals generally contain few to common radiolarians.

Preservation of radiolarians in the sections investigated from Hole $747 \mathrm{~A}$ is poor to moderate, except in the uppermost two samples placed into the Cycladophora spongothorax Zone, in which the preservation is improved. Especially in the lower portion of the sediment sequence, from Section 120$747 \mathrm{~A}-13-\mathrm{CC}$ to Sample $120-747 \mathrm{~A}-11 \mathrm{H}-1,45-47 \mathrm{~cm}$, and in the upper portion, between Samples 120-747A-6H-6, 45-47 cm, and $-6 \mathrm{H}-4,45-47 \mathrm{~cm}$, radiolarian preservation is poor and bears strongly altered radiolarian assemblages that do not allow a detailed biostratigraphic analysis.

Reworking of older radiolarian species was observed throughout the interval investigated in Hole 747A. Especially in Sample 120-747A-8H-7, 45-47 cm, significant numbers of reworked radiolarian species of Oligocene age were recognized.

Section 120-747A-13H-CC was assigned to the Stylosphaera radiosa Zone because of the rare occurrence of its nominate species. According to Abelmann (1990), the LAD of $S$. radiosa can be placed into the earliest Miocene. In the overlying sediment interval, up to Sample 120-747A-11H-6, $45-47 \mathrm{~cm}$, biostratigraphically useful species were not encountered except for the occurrences of Cyrtocapsella tetrapera and scattered $S$. radiosa (Fig. 4). Thus, no radiolarian zones could be assigned to this interval, which is marked by poorly preserved radiolarian assemblages. In its lower portion, the interval is characterized by occurrences of $S$. santaeannae and Hexastylus spp. The FO of the cosmopolitan radiolarian species Cyrtocapsella tetrapera is noted between Samples 120-747A-13H-3, 45-47 cm, and -13H-4, 45-47 cm (118.5-117 mbsf). Assuming that this FO represents the FAD of $C$. tetrapera and that the FAD of $C$. tetrapera is synchronous between the low-latitude Pacific and the Southern Ocean area studied, the interval from 118.5 to 117 mbsf can be placed in a time interval correlated with the interval from 22.2 to 22.6 $\mathrm{Ma}$, according to the stratigraphic age determination of the FAD of $C$. tetrapera by Barron et al. (1985) and Berggren et al. (1985), respectively.

Above the unzoned sections, a sediment interval corresponding to the $E$. punctatum Zone, which straddles the early/middle Miocene boundary, was recognized based on the FO of the nominate species encountered in Section 120-747A$10 \mathrm{H}-\mathrm{CC}$. It cannot be determined whether this FO represents the FAD of $E$. punctatum or whether it is linked to an improvement in radiolarian preservation that is noted at the same level. In the upper part of the E. punctatum Zone between Section 120-747A-9H-CC and Sample 120-747A$9 \mathrm{H}-8,45-47 \mathrm{~cm}$, the FOs of a series of species, such as Lamprocyrtis(?) cf. hannai, Gondwanaria deflandrei, and Hexacontium cf. enthacanthum, were noted. Either this event is related to sudden changes in paleoenvironmental conditions or a hiatus occurs at this level. The base of the middle Miocene Cycladophora humerus Zone, marked by the FAD of C. humerus, was noticed between Sample 120-747A-9H-2, $45-47 \mathrm{~cm}$, and Section 120-747A-8H-CC (75.5-77.5 mbsf).

The boundary of the $C$. humerus and Actinomma golownini zones can be placed between Section 120-747A-7H-CC and Sample $120-747 \mathrm{~A}-7 \mathrm{H}-7,45-47 \mathrm{~cm}(65.95-66 \mathrm{mbsf})$ and is marked by a disconformity. The disconformity is recognized by the first and last occurrence (LO), or the reoccurrence, of a number of species at that level (Fig. 4). This corresponds to the finding of Harwood and Majurama (this volume) that the Denticulopsis praedimorpha diatom zone is lacking because of a hiatus between Sample 120-747A-8H-1, 47-49 cm, and Section $120-747$ A-7H-CC. The A. golownini Zone, which was recognized up to the interval between Section 120-747A$6 \mathrm{H}-\mathrm{CC}$ and Sample $120-747 \mathrm{~A}-6 \mathrm{H}-6,45-47 \mathrm{~cm}$, could not be subdivided into an upper and lower subzone because $D$. megalocephalis was not encountered. The radiolarian preservation in this interval, especially in the overlying interval between Samples $120-747 \mathrm{~A}-7 \mathrm{H}-3,45-47 \mathrm{~cm}$, to $-6 \mathrm{H}-5,45-47$ $\mathrm{cm}$, is poor and the abundance of radiolarians mostly very low. For this reason, the interval between Samples 120-747A$6 \mathrm{H}-6,45-47 \mathrm{~cm}$, to $-6 \mathrm{H}-4,45-47 \mathrm{~cm}$, could not be zoned. In 
Sample 120-747A-6H-6, 45-47 cm, skeletal fragments were encountered that probably can be attributed to Cycladophora spongothorax. However, the earliest occurrence of specimens unequivocally identified as $C$. spongothorax is in Sample $120-747 \mathrm{~A}-6 \mathrm{H}-3,45-47 \mathrm{~cm}$, where the radiolarian preservation of radiolarians is improved. Thus, the boundary between the $A$. golownini and the $C$. spongothorax zones cannot be delineated accurately. Between Samples 120-747A-6H-4, $45-47 \mathrm{~cm}$, and $-6 \mathrm{H}-3,45-47 \mathrm{~cm}(52-50.5 \mathrm{mbsf})$, at the boundary between middle and upper Miocene sediments, a hiatus was recognized. The presence of this hiatus can be deduced from the absence of $A$. golownini above the interval between 52 and 50.5 mbsf. The LAD of A. golownini was placed by Lazarus (1990) at 10.3 Ma. Thus, the normal geomagnetic polarity interval that occurs above the hiatus represents the upper part of Chron $\mathrm{C} 5$, whereas the lower part of the normal interval of $\mathrm{C} 5$ is omitted.

\section{Site 748}

Site 748 is located on the Southern Kerguelen Plateau in the western part of the Raggatt Basin, east of Banzare Bank $\left(58^{\circ} 26.45^{\prime} \mathrm{S}, 78^{\circ} 58.89^{\prime} \mathrm{E}\right)$ at a water depth of $1290 \mathrm{~m}$ (Fig. 1). Of the three holes drilled at this site, lower and middle Miocene sediments were only recovered in Hole 748B. In this study, radiolarians were investigated in the interval between Sections $120-748 \mathrm{~B}-8 \mathrm{H}-\mathrm{CC}$ and $-5 \mathrm{H}-5$, which represents the early to middle Miocene time interval. Radiolarian ranges and abundance patterns for the the sections studied are shown in Table 3 and Figure 5. In contrast to Hole $747 \mathrm{~A}$, radiolarians are generally common to abundant and their preservation is good throughout the investigated sections.

Hole 748B recovered a long lower Miocene sequence, whereas middle Miocene sediments are mostly lacking as a result of a hiatus that occurs between Sample 120-748B-6H-1, $45-47 \mathrm{~cm}$, and Section 120-748B-5H-CC (38.6 -38.1 mbsf). This hiatus is estimated to span the time interval from 16.5 to $11 \mathrm{Ma}$.

The lowermost part of the sediment section, above Section $120-748 \mathrm{~B}-8 \mathrm{H}-\mathrm{CC}$ can be placed into the Stylosphaera radiosa Zone, characterized by the consistent occurrence of the nominate species. Co-occurring characteristic radiolarian species in this zone are $S$. coronata laevis, Actinomma holtedahli, Lithomelissa robusta, Lychnocanoma conica, Spongodiscus craticulatus, Corythospyris fiscella, Velicucullus cf. oddgurneri, and Velicucullus altus. The FO of Cenosphaera sp. A, V. cf. oddgurneri, Prunopyle sp. A, Ceratocyrtis mashae and $C$. fiscella is marked between Section $120-748 \mathrm{~B}-8 \mathrm{H}-\mathrm{CC}$ and Sample 120-748B-8H-6, 45-47 cm. Between Samples 120-748B-8H-5, 45-47 cm, and $-8 \mathrm{H}-4$, $45-47 \mathrm{~cm}$, the FOs of Eucyrtidium cienkowskii and Prunopyle titan and the LOs of $A$. holtedahli and A. medusa are noted. This radiolarian occurrence pattern probably indicates the presence of a hiatus at this level.

The boundary of the $S$. radiosa and Cycladophora antiqua zones, which is marked by the LAD of $S$. radiosa and the FADs of $C$. antiqua and Cyrtocapsella tetrapera, was found between Samples 120-748B-8H-3, 45-47 cm, and -8H-2, $45-47 \mathrm{~cm}$. At the same level, Dendrospyris stabilis, Cycladophora conica, and Stylosphaera sp. C are recognized for the first time and $S$. coronata laevis for the last time. According to the radiolarian biostratigraphic results and geomagnetic polarity pattern, it is suggested that a disconformity occurs at this level that probably omits Subchron C6A and part of Subchron C6B.

Between Samples 120-748B-8H-2, 45-47 cm, and -8H-1, $45-47 \mathrm{~cm}$, the FOs of $C$. golli regipileus, Lithomelissa cf. ehrenbergii, and Gondwanaria deflandrei are recorded. This pattern indicates that a hiatus occurs at this level that omits the early Miocene Cyrtocapsella longithorax Zone, and possibly the upper portion of the $C$. antiqua Zone and the basal portion of the Cycladophora golli regipileus Zone. Considering the geomagnetic polarity pattern, a time interval spanning part of Subchron C6A and the lower part of Chron C6 may be included in the hiatus. Within the sediment interval assigned to the Cycladophora golli regipileus Zone, the FOs of a series of radiolarian taxa are noted.

Between Samples 120-748B-6H-2, 45-47 cm, and - $6 \mathrm{H}-1$, $45-47 \mathrm{~cm}$, is the boundary between the $C$. golli regipileus and Eucyrtidium punctatum zones, recognized by the FO of $E$. punctatum. This level is marked by a hiatus, according to diatom biostratigraphic results (Harwood and Maruyama, this volume).

A further hiatus that omits a large portion of the $E$. punctatum, the Cycladophora humerus and the Actinomma golownini zones, and thus a major part of the middle Miocene, was recognized in the interval between Sample 120-748B$6 \mathrm{H}-1,45-47 \mathrm{~cm}$, and Section 120-748B-5H-CC (around 38.3 mbsf), because of the FO of Cycladophora spongothorax at this level. $C$. spongothorax is the nominate species of a radiolarian zone that correlates with a time spanning the late middle Miocene from $\sim 11.8 \mathrm{Ma}$ to the early late Miocene (Abelmann, 1990; Lazarus, 1990; Gersonde et al., 1990). The age range of the hiatus can be interpreted to be older than 10.3 Ma. This age represents the LAD of $A$. golownini, a species that commonly occurs above the hiatus. Therefore, the disconformity at 38.3 mbsf spans approximately the time interval from $\sim 16.5$ to $\sim 11 \mathrm{Ma}$ (for further remarks, the reader is referred to the "Discussion" section, this chapter).

\section{Site 751}

Site 751 was cored in the central part of the Raggatt Basin on the Southern Kerguelen Plateau $\left(57^{\circ} 43.56^{\prime} \mathrm{S}, 79^{\circ} 48.89^{\prime} \mathrm{E}\right)$, in a water depth of $1633.8 \mathrm{~m}$. At Site 751 one hole with a 166-m-thick middle lower Miocene to Pleistocene sequence of mixed biosiliceous and calcareous ooze was recovered. In this study, lower to middle Miocene sediment sequences were investigated between Sections $120-751 \mathrm{~A}-18 \mathrm{H}-\mathrm{CC}$ and $-10 \mathrm{H}-$ CC. For ranges and abundance patterns of radiolarians of the studied sections, the reader is referred to Table 4 and Figures 6 and 7. Generally, the preservation of the radiolarian assemblages studied from Hole 751A is good to moderate. Radiolarians generally occur in high abundances. An exception is the interval in the $E$. punctatum Zone between Samples $120-751 \mathrm{~A}-14 \mathrm{H}-1,98-100 \mathrm{~cm}$, and $-13 \mathrm{H}-3,32-36 \mathrm{~cm}$, in which the abundance of radiolarians is low. In particular, Samples $120-751 \mathrm{~A}-14 \mathrm{H}-1,98-100 \mathrm{~cm}$, and $-13 \mathrm{H}-4,98-100 \mathrm{~cm}$, are barren in radiolarians because of the mass occurrence of the diatom species Actinocyclus ingens.

The basal portion of the sediments recovered in Hole $751 \mathrm{~A}$ (Section 120-751A-18H-CC to Sample 120-751A-18H-4, 98$100 \mathrm{~cm}$ ) can be assigned to the middle early Miocene Cyrtocapsella longithorax Zone because of the occurrence of the nominate species and the absence of Cycladophora golli regipileus. A series of radiolarian species including Ceratocyrtis stigi, Prunopyle sp. B, Prunopyle hayesi, Carpocanarium papillosum, Calocyclas cf. semipolita, Cycladophora sp., Cenosphaera sp. A, and Spongodiscus craticulatus appear for the first time between Samples 120-751A-18H-5, 98-100 cm, and $-18 \mathrm{H}-4,98-100 \mathrm{~cm}$. This occurrence pattern may suggest that a disconformity occurs at this level within the $C$. longitho$\operatorname{rax}$ Zone.

The boundary between the $C$. longithorax and Cycladophora golli regipileus zones, marked by the FAD of $C$. golli regipileus, was recognized between Samples 120-751A-18H- 
Table 2. Stratigraphic occurrences of radiolarian species in the early to middle Miocene, Hole 747A.

\begin{tabular}{|c|c|c|c|c|c|c|c|c|c|c|c|c|c|c|c|c|c|c|c|c|c|c|}
\hline Zones & & $S$. radios & & & & & & & & & & & Unzoned & & & & & & & & & \\
\hline $\begin{array}{l}\text { Core, section } \\
\text { Interval (cm) }\end{array}$ & $\begin{array}{l}13 \mathrm{H}- \\
\mathrm{CC}\end{array}$ & $\begin{array}{l}13 \mathrm{H}-6 \\
45-47\end{array}$ & $\begin{array}{l}13 \mathrm{H}-5 \\
45-47 \\
\end{array}$ & $\begin{array}{l}13 \mathrm{H}-4 \\
45-47\end{array}$ & $\begin{array}{l}13 \mathrm{H}-3 \\
45-47\end{array}$ & $\begin{array}{l}13 \mathrm{H}-2 \\
45-47\end{array}$ & $\begin{array}{l}13 \mathrm{H}-1 \\
45-47\end{array}$ & $\begin{array}{l}12 \mathrm{H}- \\
\mathrm{CC}\end{array}$ & $\begin{array}{l}12 \mathrm{H}-6 \\
45-47\end{array}$ & $\begin{array}{l}12 \mathrm{H}-5 \\
45-47\end{array}$ & $\begin{array}{l}12 \mathrm{H}-4 \\
45-47\end{array}$ & $\begin{array}{l}12 \mathrm{H}-3 \\
45-47\end{array}$ & $\begin{array}{l}12 \mathrm{H}-2 \\
45-47\end{array}$ & $\begin{array}{l}12 \mathrm{H}-1 \\
45-47\end{array}$ & $\begin{array}{l}11 \mathrm{H}- \\
\mathrm{CC}\end{array}$ & $\begin{array}{l}11 \mathrm{H}-7 \\
45-47\end{array}$ & $\begin{array}{l}11 \mathrm{H}-6 \\
45-47\end{array}$ & $\begin{array}{l}111 \mathrm{H}-5 \\
45-47\end{array}$ & $\begin{array}{l}11 \mathrm{H}-4 \\
45-47\end{array}$ & $\begin{array}{l}11 \mathrm{H}-3 \\
45-47\end{array}$ & $\begin{array}{l}11 \mathrm{H}-2 \\
45-47\end{array}$ & $\begin{array}{l}11 \mathrm{H}-1 \\
45-47\end{array}$ \\
\hline $\begin{array}{l}\text { Abundance } \\
\text { Preservation }\end{array}$ & $\begin{array}{l}F \\
P\end{array}$ & $\begin{array}{l}\mathrm{R} \\
\mathrm{P}\end{array}$ & $\begin{array}{l}\mathrm{R} \\
\mathrm{P}\end{array}$ & $\begin{array}{l}\mathrm{R} \\
\mathrm{P}\end{array}$ & $\begin{array}{l}\mathrm{R} \\
\mathrm{VP}\end{array}$ & $\begin{array}{l}\mathrm{R} \\
\mathrm{P}\end{array}$ & $\begin{array}{l}\mathrm{R} \\
\mathrm{P}\end{array}$ & $\begin{array}{l}\mathrm{F} \\
\mathrm{P}\end{array}$ & $\begin{array}{l}\mathrm{F} \\
\mathrm{P}\end{array}$ & $\begin{array}{l}T \\
P\end{array}$ & $\begin{array}{l}F \\
P\end{array}$ & $\begin{array}{l}T \\
P\end{array}$ & $\begin{array}{l}\mathrm{R} \\
\mathrm{P}\end{array}$ & $\begin{array}{l}R \\
P\end{array}$ & $\begin{array}{l}\mathrm{F} \\
\mathrm{P}\end{array}$ & $\begin{array}{c}\text { T-R } \\
P\end{array}$ & $\underset{M \cdot P}{C}$ & $\begin{array}{l}\mathrm{T} \\
\mathrm{P}\end{array}$ & $\begin{array}{l}\mathrm{T} \\
\mathrm{P}\end{array}$ & $\begin{array}{l}\mathrm{T} \\
\mathrm{P}\end{array}$ & $\begin{array}{l}\mathrm{T} \\
\mathrm{P}\end{array}$ & $\begin{array}{l}\mathrm{R} \\
\mathrm{P}\end{array}$ \\
\hline $\begin{array}{l}\text { Hexastylus spp. } \\
\text { Actinomma sp. B } \\
\text { Heliodiscus (?) sp. B } \\
\text { Stylosphaera radiosa } \\
\text { Stylatractus santaeannae }\end{array}$ & $\begin{array}{l}\mathrm{C} \\
\mathrm{C} \\
\mathrm{R} \\
\mathrm{R} \\
\mathrm{R}\end{array}$ & $\begin{array}{l}\mathrm{R} \\
\mathrm{R} \\
\mathrm{R}\end{array}$ & $\begin{array}{l}\mathrm{F} \\
\mathrm{R} \\
\mathrm{R}\end{array}$ & $R$ & $R$ & $\begin{array}{c}\mathrm{R}-\mathrm{F} \\
\mathrm{R}\end{array}$ & $\begin{array}{l}\mathrm{R} \\
\mathrm{R}\end{array}$ & $\begin{array}{l}\mathrm{C} \\
\mathrm{C} \\
\mathrm{R} \\
\mathrm{T} \\
\mathrm{T}\end{array}$ & $\begin{array}{l}\mathrm{C} \\
\mathrm{R} \\
\mathrm{T} \\
\mathrm{R}\end{array}$ & $\mathrm{T}$ & $\begin{array}{l}\mathrm{C} \\
\mathrm{T} \\
\mathrm{T}\end{array}$ & T & $\begin{array}{l}\mathrm{C} \\
\mathrm{R}\end{array}$ & $\begin{array}{l}\mathrm{T} \\
\mathrm{R}\end{array}$ & $\begin{array}{l}\mathrm{F} \\
\mathrm{C} \\
\mathrm{R}\end{array}$ & $T$ & $\begin{array}{l}\mathrm{R} \\
\mathrm{C} \\
\mathrm{R} \\
\mathrm{T}\end{array}$ & $\mathrm{T}$ & & T & & R \\
\hline $\begin{array}{l}\text { Cenosphaera sp. } \\
\text { Siphocampe sp. } \\
\text { Orosphaera sp.? } \\
\text { Lychnocanoma conica } \\
\text { Stylosphaera sp. B }\end{array}$ & & $\begin{array}{l}\mathrm{F} \\
\mathrm{R} \\
\mathrm{R} \\
\mathrm{R} \\
\mathrm{R}\end{array}$ & $\begin{array}{l}\mathrm{C} \\
\mathrm{R} \\
\mathrm{R} \\
\mathrm{R} \\
\mathrm{R}\end{array}$ & $\begin{array}{l}\mathrm{C} \\
\mathrm{R} \\
\mathrm{R}\end{array}$ & $\begin{array}{l}\text { C } \\
T \\
T \\
T\end{array}$ & $\begin{array}{l}\mathrm{C} \\
\mathrm{T} \\
\mathrm{T} \\
\mathrm{R}\end{array}$ & $\begin{array}{l}\mathrm{F} \cdot \mathrm{C} \\
\mathrm{T} \\
\mathrm{R}\end{array}$ & & & $\stackrel{\text { R-F }}{\mathrm{T}}$ & & $\begin{array}{l}\mathrm{F} \\
\mathrm{T}\end{array}$ & & $\begin{array}{l}C \\
T\end{array}$ & & $\begin{array}{l}\mathrm{T} \\
\mathrm{T} \\
\mathrm{R}\end{array}$ & & $\begin{array}{l}\mathrm{F} \\
\mathrm{T} \\
\mathrm{T}\end{array}$ & $\begin{array}{l}\mathrm{F}-\mathrm{C} \\
\mathrm{R} \\
\end{array}$ & $\begin{array}{l}\mathrm{F} \\
\mathrm{T}\end{array}$ & $\begin{array}{l}\text { R-F } \\
T \\
\end{array}$ & $\begin{array}{l}\mathrm{F} \\
\mathrm{R} \\
\mathrm{R}\end{array}$ \\
\hline $\begin{array}{l}\text { Lamprocyclas sp. } \\
\text { Carpocanarium papillosum } \\
\text { Cycladophora sp. } \\
\text { Stylosphaera sp. } \\
\text { Cyrtocapsella tetrapera }\end{array}$ & & $T$ & $\begin{array}{l}\mathrm{T} \\
\mathrm{T}\end{array}$ & $\begin{array}{c}R \\
R \\
F-C\end{array}$ & $\begin{array}{l}T \\
T \\
T \\
T \\
R\end{array}$ & $\begin{array}{l}\mathrm{R} \\
\mathrm{T} \\
\mathrm{F} \\
\mathrm{T}\end{array}$ & $\begin{array}{l}\mathrm{T} \\
\mathrm{T} \\
\mathrm{T}\end{array}$ & $\mathrm{R}$ & & $\mathrm{T}$ & F & $\begin{array}{l}T \\
T\end{array}$ & $\mathrm{R}$ & $\begin{array}{l}T \\
R\end{array}$ & $\mathbf{R}$ & $\begin{array}{l}\mathrm{T} \\
\mathrm{T}\end{array}$ & $\begin{array}{l}R \\
R\end{array}$ & $\begin{array}{l}\mathrm{T} \\
\mathrm{T}\end{array}$ & $\mathrm{T}$ & $\mathrm{T}$ & $\begin{array}{l}\mathrm{T} \\
\mathrm{T}\end{array}$ & $T$ \\
\hline $\begin{array}{l}\text { Corythospyris fiscella } \\
\text { Prunopyle hayesi } \\
\text { Amphistylus angelinus } \\
\text { Spongodiscus craticulatus } \\
\text { Stauroxiphos communis }\end{array}$ & & & $\mathrm{T}$ & & $\begin{array}{l}T \\
T\end{array}$ & $\begin{array}{l}T \\
T \\
R\end{array}$ & $T$ & $\begin{array}{l}\mathrm{T} \\
\mathrm{R} \\
\mathrm{T}\end{array}$ & & $\mathrm{T}$ & $\begin{array}{l}\mathrm{R} \\
\mathrm{T} \\
\mathrm{R}\end{array}$ & & $\begin{array}{l}\mathrm{T} \\
\mathrm{R} \\
\mathrm{R} \\
\mathrm{F}\end{array}$ & & R & & $\begin{array}{l}\mathrm{R} \\
\mathrm{R} \\
\mathrm{R}\end{array}$ & & & & & \\
\hline $\begin{array}{l}\text { Dendrospyris stabilis } \\
\text { Sylosphaera neptumus } \\
\text { Acrosphaera spp. } \\
\text { Prunopyle sp. B } \\
\text { Ceratocyrtis stigi }\end{array}$ & & & & $\mathrm{T}$ & & $\mathrm{T}$ & & & & & & & & & & & $\begin{array}{c}T \\
R \\
R-F \\
T \\
T \\
T\end{array}$ & & & & & \\
\hline $\begin{array}{l}\text { Eucyrtidium sp. B } \\
\text { Eucritidium punctatum } \\
\text { Sylosphaera sp. A } \\
\text { Cycladophora golli regipileus } \\
\text { Cyrtocapsella longithorax }\end{array}$ & & & & & & & & & & & & & & & & & & & & & & \\
\hline $\begin{array}{l}\text { Eucyrtidium cienkowskii gr. } \\
\text { Pterocanium sp. } \\
\text { Lamprocyrtis (?) cf. hannai } \\
\text { Gondwanaria deflandrei } \\
\text { Hexacontium cf. enthacanthum }\end{array}$ & & & & & & & & & & & & & & & & & & & & & & \\
\hline $\begin{array}{l}\text { Druppatractus hastatus } \\
\text { Prinopyle sp. D } \\
\text { Lychnocanoma sp. B } \\
\text { Cycladophora humenus } \\
\text { Eucyridium sp. A }\end{array}$ & & & & & & & & & & & & & & & & & & & & & & \\
\hline $\begin{array}{l}\text { Heliodiscus sp. A } \\
\text { Velicucullus cf. oddgumeri } \\
\text { Spongomelissa dilli } \\
\text { Peripyramis circumtexa } \\
\text { Dendrospyris rhodospyroides }\end{array}$ & & & & & & & & & & & & & & & & & & & & & & \\
\hline $\begin{array}{l}\text { Stylodictya validispina } \\
\text { Spongopyle osculosa } \\
\text { Trissocyclid sp. A } \\
\text { Actinomma golownini } \\
\text { Eucyrtidium calvertense }\end{array}$ & & & & & & & & & & & & & & & & & & & & & & \\
\hline $\begin{array}{l}\text { Cornutella profinda } \\
\text { Cormutella clathrata } \\
\text { Cyntocapsella cormuta } \\
\text { Antarctissa deflandrei } \\
\text { Rhopalodictyum sp. }\end{array}$ & & & & & & & & 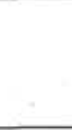 & & & & & & & & & & & & & & \\
\hline $\begin{array}{l}\text { Collosphaerid sp. B } \\
\text { Lithocarpium polyacantha } \\
\text { Cycladophora spongothorax }\end{array}$ & & & & & & & & & & & & & & & & & & & & & & \\
\hline
\end{tabular}

Note. Abundance: $\mathrm{A}=$ abundant, $\mathrm{C}=$ common, $\mathrm{F}=$ few, $\mathrm{R}=$ rare, and $\mathrm{T}=$ trace. Preservation: $\mathrm{G}=$ good, $\mathrm{M}=$ moderate, and $\mathrm{P}=$ poor. 
Table 2 (continued).

\begin{tabular}{|c|c|c|c|c|c|c|c|c|c|c|c|c|c|c|c|c|c|c|c|c|c|c|c|}
\hline \multirow{2}{*}{$\begin{array}{l}\text { Zones } \\
\text { Core, section } \\
\text { Interval (cm) }\end{array}$} & \multicolumn{14}{|c|}{ E. punctatum } & \multicolumn{9}{|c|}{ Cycladophora humenus } \\
\hline & $\begin{array}{l}10 \mathrm{H}- \\
\mathrm{CC}\end{array}$ & $\begin{array}{l}10 \mathrm{H}-6 \\
45-47\end{array}$ & $\begin{array}{l}10 \mathrm{H}-5 \\
45-47\end{array}$ & $\begin{array}{l}10 \mathrm{H}-4 \\
45-47\end{array}$ & $\begin{array}{l}10 \mathrm{H}-3 \\
45-47\end{array}$ & $\begin{array}{l}10 \mathrm{H}-2 \\
45-47\end{array}$ & $\begin{array}{l}10 \mathrm{H}-1 \\
45-47\end{array}$ & $\begin{array}{l}9 \mathrm{H}- \\
\mathrm{CC}\end{array}$ & $\begin{array}{l}9 \mathrm{H}-8 \\
45-47\end{array}$ & $\begin{array}{l}9 \mathrm{H}-6 \\
45-47\end{array}$ & $\begin{array}{l}9 \mathrm{H}-5 \\
45-47\end{array}$ & $\begin{array}{l}9 \mathrm{H}-4 \\
45-47\end{array}$ & $\begin{array}{c}9 \mathrm{H}-3 \\
45-47\end{array}$ & $\begin{array}{l}9 \mathrm{H}-2 \\
45-47\end{array}$ & \begin{tabular}{|l}
$8 \mathrm{H}-$ \\
$\mathrm{CC}$
\end{tabular} & $\begin{array}{l}8 \mathrm{H}-7 \\
45-47\end{array}$ & $\begin{array}{l}8 \mathrm{H}-6 \\
45-47\end{array}$ & $\begin{array}{l}8 \mathrm{H}-5 \\
45-47\end{array}$ & $\begin{array}{l}8 \mathrm{H}-4 \\
45-47\end{array}$ & $\begin{array}{l}8 \mathrm{H}-3 \\
45-47\end{array}$ & $\begin{array}{l}8 \mathrm{H}-2 \\
45-47\end{array}$ & $\begin{array}{c}8 \mathrm{H}-1 \\
45-47\end{array}$ & $\begin{array}{l}7 \mathrm{H}- \\
\mathrm{CC}\end{array}$ \\
\hline $\begin{array}{l}\text { Abundance } \\
\text { Preservation }\end{array}$ & $\underset{M-P}{C}$ & $\underset{\mathrm{M}-\mathrm{P}}{\mathrm{C}}$ & $\underset{\mathrm{M}-\mathrm{P}}{\mathrm{F}}$ & $\begin{array}{l}\mathrm{F} \\
\mathrm{P}\end{array}$ & $\underset{\mathrm{M} \cdot \mathrm{P}}{\mathrm{F}}$ & $\begin{array}{l}\mathrm{F} \\
\mathrm{M}\end{array}$ & $\begin{array}{l}\mathrm{F} \\
\mathrm{P}\end{array}$ & $\begin{array}{l}\mathrm{C} \\
\mathrm{M}\end{array}$ & $\underset{\mathrm{M}-\mathrm{P}}{\mathrm{C}}$ & $\begin{array}{l}\mathrm{C} \\
\mathrm{M}\end{array}$ & B & B & B & B & $\begin{array}{l}\mathrm{A} \\
\mathrm{M}\end{array}$ & $\begin{array}{l}\mathrm{F} \\
\mathrm{M}\end{array}$ & $\begin{array}{l}\mathrm{R} \\
\mathrm{M}\end{array}$ & $\begin{array}{l}\mathrm{T} \\
\mathrm{P}\end{array}$ & $\begin{array}{l}\mathrm{R} \\
\mathrm{P}\end{array}$ & $\begin{array}{l}\mathrm{T} \\
\mathrm{P}\end{array}$ & $\begin{array}{l}\mathrm{R} \\
\mathrm{P}\end{array}$ & $\begin{array}{l}\mathrm{R} \\
\mathrm{P}\end{array}$ & $\begin{array}{l}\mathrm{R} \\
\mathrm{P}\end{array}$ \\
\hline $\begin{array}{l}\text { Hexastylus spp. } \\
\text { Actinomma sp. B } \\
\text { Heliodiscus (?) sp. B } \\
\text { Stylosphaera radiosa } \\
\text { Stylatractus santaeannae }\end{array}$ & $\underset{\mathrm{R}-\mathrm{F}}{\mathrm{C}}$ & $\begin{array}{l}\mathrm{F} \\
\mathrm{R}\end{array}$ & & & & & & $\begin{array}{l}\mathrm{C} \\
\mathrm{R}\end{array}$ & $\mathrm{F}$ & $\mathrm{F}$ & & & & & $\mathrm{T}$ & $\begin{array}{l}\mathrm{T} \\
\mathrm{R} \\
\mathrm{R}\end{array}$ & $\mathrm{R}$ & & & & & & \\
\hline $\begin{array}{l}\text { Cenosphaera sp. } \\
\text { Siphocampe sp. } \\
\text { Orosphaera sp. ? } \\
\text { Lychnocanoma conica } \\
\text { Stylosphaera sp. B }\end{array}$ & $\mathrm{F}$ & $\mathbf{R}$ & & $\mathrm{T}$ & $\mathrm{T}$ & $\mathrm{T}$ & $\begin{array}{l}R \\
T\end{array}$ & $\mathbf{R}$ & & $\mathrm{T}$ & & & & & $\mathrm{T}$ & & $\mathrm{T}$ & & $\mathrm{T}$ & & & & \\
\hline $\begin{array}{l}\text { Lamprocyclas sp. } \\
\text { Carpocanarium papillosum } \\
\text { Cycladophora sp. } \\
\text { Stylosphaera sp. C } \\
\text { Cyrtocapsella tetrapera }\end{array}$ & $\mathrm{R}$ & R-F & R-F & $\mathbf{R}$ & $\mathbf{R}$ & $\mathbf{R}$ & $\mathrm{T}$ & $\mathrm{F}$ & $\mathrm{F}$ & T & & & & & F & $\mathrm{T}$ & $\begin{array}{l}\mathrm{R} \\
\mathrm{C}\end{array}$ & $\mathrm{T}$ & $\begin{array}{l}\text { R } \\
\text { F }\end{array}$ & $\begin{array}{l}\mathrm{T} \\
\mathrm{T}\end{array}$ & $T$ & $\begin{array}{l}\mathrm{T} \\
\mathrm{T}\end{array}$ & $\mathbf{R}$ \\
\hline $\begin{array}{l}\text { Corythospyris fiscella } \\
\text { Prunopyle hayesi } \\
\text { Amphistylus angelinus } \\
\text { Spongodiscus craticulatus } \\
\text { Stauroxiphos communis }\end{array}$ & $\begin{array}{l}\mathrm{R} \\
\mathrm{R} \\
\mathrm{R}\end{array}$ & $\begin{array}{c}\mathrm{R} \\
\mathrm{R} \\
\mathrm{R}-\mathrm{F}\end{array}$ & $\begin{array}{l}\mathrm{R} \\
\mathrm{R} \\
\mathrm{R}\end{array}$ & $\begin{array}{l}\mathrm{R} \\
\mathrm{R}\end{array}$ & $\begin{array}{l}\mathrm{T} \\
\mathrm{R}\end{array}$ & $\begin{array}{l}\mathrm{T} \\
\mathrm{T} \\
\mathrm{T} \\
\mathrm{R}\end{array}$ & $\begin{array}{l}\mathrm{T} \\
\mathrm{T} \\
\mathrm{R}\end{array}$ & $\begin{array}{l}\mathrm{T} \\
\mathrm{R} \\
\mathrm{T}\end{array}$ & $\begin{array}{l}\mathrm{R} \\
\mathrm{F}\end{array}$ & $\begin{array}{l}\mathrm{T} \\
\mathrm{R} \\
\mathrm{R}\end{array}$ & & & & $\mathbf{R}$ & $\mathbf{F}$ & $\mathrm{R}$ & $\mathbf{R}$ & & $\mathrm{T}$ & & & & $\mathbf{R}$ \\
\hline $\begin{array}{l}\text { Dendrospyris stabilis } \\
\text { Stylosphaera neptumus } \\
\text { Acrosphaera spp. } \\
\text { Prunopyle sp. B } \\
\text { Ceratocyrtis stigi }\end{array}$ & $\begin{array}{l}\mathrm{T} \\
\mathrm{R}\end{array}$ & $\begin{array}{l}\mathrm{T} \\
\mathrm{R} \\
\mathrm{T}\end{array}$ & $\begin{array}{c}\mathrm{T} \\
\mathrm{R}-\mathrm{F}\end{array}$ & $\mathbf{R}$ & & $\begin{array}{l}\mathrm{R} \\
\mathrm{R} \\
\mathrm{T} \\
\mathrm{T}\end{array}$ & $\mathrm{T}$ & $\begin{array}{l}\mathrm{T} \\
\mathrm{F}\end{array}$ & $\begin{array}{l}\mathrm{T} \\
\mathrm{R}\end{array}$ & $\mathrm{R}$ & & & & & $\mathbf{R}$ & $\begin{array}{l}\mathrm{R} \\
\mathrm{R}\end{array}$ & $\begin{array}{l}\mathrm{R} \\
\mathrm{R}\end{array}$ & & $\mathbf{R}$ & $\mathrm{T}$ & $\mathrm{T}$ & $\mathrm{R}$ & $\mathrm{T}$ \\
\hline $\begin{array}{l}\text { Eucyrtidium sp. B } \\
\text { Eucyrtidium punctatum } \\
\text { Stylosphaera sp. A } \\
\text { Cycladophora golli regipileus } \\
\text { Cyrtocapsella longithorax }\end{array}$ & $\begin{array}{l}\mathrm{R} \\
\mathrm{T} \\
\mathrm{T}\end{array}$ & $\begin{array}{l}R \\
R \\
R\end{array}$ & R-F & $\mathbf{R}$ & $\begin{array}{l}\mathrm{R} \\
\mathrm{T}\end{array}$ & $\begin{array}{l}\mathrm{R} \\
\mathrm{T}\end{array}$ & $\begin{array}{l}\mathrm{R} \\
\mathrm{T}\end{array}$ & $\begin{array}{l}\mathrm{R} \\
\mathrm{R} \\
\mathrm{F} \\
\mathrm{R}\end{array}$ & $\begin{array}{l}\mathrm{R} \\
\mathrm{F} \\
\mathrm{R}\end{array}$ & $\begin{array}{l}\mathrm{T} \\
\mathrm{R} \\
\mathrm{R}\end{array}$ & & & & s & $\begin{array}{l}\mathrm{T} \\
\mathrm{R}\end{array}$ & & & $\mathrm{T}$ & $\begin{array}{l}\mathrm{R} \\
\mathrm{T}\end{array}$ & $\mathbf{R}$ & 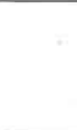 & $\mathrm{T}$ & \\
\hline $\begin{array}{l}\text { Eucyrtidium cienkowskii gr. } \\
\text { Plerocanium sp. } \\
\text { Lamprocyrtis (?) cf. hannai } \\
\text { Gondwanara deflandrei } \\
\text { Hexacontium ef. enthacanthum }\end{array}$ & & & R-F & $\mathbf{R}$ & R & $\mathbf{R}$ & $\mathbf{R}$ & $\begin{array}{l}\mathrm{R} \\
\mathrm{T}\end{array}$ & $\begin{array}{l}\mathrm{F} \\
\mathrm{T} \\
\mathrm{F} \\
\mathrm{T} \\
\mathrm{T}\end{array}$ & $\begin{array}{l}\mathrm{R} \\
\mathrm{R} \\
\mathrm{T} \\
\mathrm{R}\end{array}$ & & & & & $\begin{array}{l}\mathrm{R} \\
\mathrm{F} \\
\mathrm{T} \\
\mathrm{R}\end{array}$ & & $\begin{array}{l}\mathrm{T} \\
\mathrm{T} \\
\mathrm{R}\end{array}$ & & $\mathbf{R}$ & $\mathbf{R}$ & $\mathbf{R}$ & $\mathrm{T}$ & $\begin{array}{l}\mathrm{R} \\
\mathrm{T} \\
\mathrm{R} \\
\mathrm{R}\end{array}$ \\
\hline $\begin{array}{l}\text { Druppatractus hastatus } \\
\text { Prunopyle sp. D } \\
\text { Lychnocanoma sp. B } \\
\text { Cycladophora humerus } \\
\text { Eucyrtidium sp. A }\end{array}$ & & & $\mathrm{T}$ & $\mathrm{T}$ & & $\begin{array}{l}\mathrm{R} \\
\mathrm{T}\end{array}$ & $\begin{array}{l}\mathrm{R} \\
\mathrm{T}\end{array}$ & & $\mathbf{R}$ & $\begin{array}{l}\mathrm{R} \\
\mathrm{T} \\
\mathrm{T} \\
\mathrm{T} \\
\mathrm{F}\end{array}$ & $\mathrm{R}$ & & & & $\begin{array}{l}\mathrm{F} \\
\mathrm{R} \\
\mathrm{T} \\
\mathrm{F}\end{array}$ & & $\begin{array}{l}\mathrm{T} \\
\mathrm{R} \\
\mathrm{T} \\
\mathrm{R}\end{array}$ & $\mathrm{T}$ & $\begin{array}{l}\mathrm{R} \\
\mathrm{T} \\
\mathrm{T}\end{array}$ & $\mathbf{F}$ & $\begin{array}{l}\mathrm{R} \\
\mathrm{R}\end{array}$ & $\begin{array}{l}T \\
\mathrm{R} \\
\mathrm{T}\end{array}$ & $\begin{array}{l}R \\
R\end{array}$ \\
\hline $\begin{array}{l}\text { Heliodiscus sp. A } \\
\text { Velicucullus cf. oddgurneri } \\
\text { Spongomelissa dilli } \\
\text { Peripyramis circumtexa } \\
\text { Dendrospyris rhodospyroides }\end{array}$ & & & & & & & & & & & & & & & $\begin{array}{l}\mathrm{T} \\
\mathrm{T}\end{array}$ & & $\mathrm{T}$ & & $\mathrm{T}$ & & $\begin{array}{l}\mathrm{T} \\
\mathrm{R}\end{array}$ & $\mathrm{T}$ & $\begin{array}{l}\mathrm{T} \\
\mathrm{R} \\
\mathrm{R}\end{array}$ \\
\hline $\begin{array}{l}\text { Stylodictya validispina } \\
\text { Spongopyle osculosa } \\
\text { Trissocyclid sp. A } \\
\text { Actinomma golownini } \\
\text { Eucyrtidium calvertense }\end{array}$ & : & & & & & & & & $=$ & & & & & & & & & & & & & & $\begin{array}{l}\mathrm{R} \\
\mathrm{R} \\
\mathrm{R}\end{array}$ \\
\hline $\begin{array}{l}\text { Comutella profunda } \\
\text { Comuetlla clathrata } \\
\text { Cyrocapsella cormuta } \\
\text { Antarctissa defandrei } \\
\text { Rhopalodictyum sp. }\end{array}$ & & & & & & & & & & & & & & & & & & & & & & & \\
\hline $\begin{array}{l}\text { Collosphaerid sp. B } \\
\text { Lithocarpium polyacantha } \\
\text { Cycladophora spongothorax }\end{array}$ & & & & & & & & & & & & & & & & & & & & & & & \\
\hline
\end{tabular}




\begin{tabular}{|c|c|c|c|c|c|c|c|c|c|c|c|c|c|}
\hline \multirow{2}{*}{$\begin{array}{l}\text { Zones } \\
\text { Core, section } \\
\text { Interval (cm) } \\
\text { Abundance } \\
\text { Preservation }\end{array}$} & \multicolumn{8}{|c|}{ Actinomma golownini } & \multicolumn{3}{|c|}{ Unzoned } & \multicolumn{2}{|c|}{ C. spongothorax } \\
\hline & $\begin{array}{c}7 \mathrm{H}-7 \\
45-47 \\
R \\
\mathrm{M}\end{array}$ & $\begin{array}{c}7 \mathrm{H}-6 \\
45-47 \\
R \\
\mathrm{M}-\mathrm{P}\end{array}$ & $\begin{array}{c}7 \mathrm{H}-5 \\
45-47 \\
\mathrm{~F} \\
\mathrm{M}-\mathrm{G}\end{array}$ & $\begin{array}{c}7 \mathrm{H}-4 \\
45-47 \\
\mathrm{~F} \\
\mathrm{P}\end{array}$ & $\begin{array}{c}7 \mathrm{H}-3 \\
45-47 \\
\mathrm{~T} \\
\mathrm{P}\end{array}$ & $\begin{array}{c}7 \mathrm{H}-2 \\
45-47 \\
\mathrm{~T} \\
\mathrm{P}\end{array}$ & $\begin{array}{c}7 \mathrm{H}-1 \\
45-47 \\
\mathrm{~T} \\
\mathrm{P}\end{array}$ & $\begin{array}{l}6 \mathrm{H}- \\
\mathrm{CC} \\
\mathrm{F} \\
\mathrm{M}\end{array}$ & $\begin{array}{c}6 \mathrm{H}-6 \\
45-47 \\
F \\
P\end{array}$ & $\begin{array}{c}6 \mathrm{H}-5 \\
45-47 \\
\mathrm{~T} \\
P\end{array}$ & $\begin{array}{c}6 \mathrm{H}-4 \\
45-47 \\
F \\
P\end{array}$ & $\begin{array}{c}6 \mathrm{H}-3 \\
45-47 \\
\mathrm{C} \\
\mathrm{G}\end{array}$ & $\begin{array}{c}6 \mathrm{H}-2 \\
45-47 \\
C \\
G\end{array}$ \\
\hline $\begin{array}{l}\text { Hexastylus spp. } \\
\text { Actinomma sp. B } \\
\text { Heliodiscus (?) sp. B } \\
\text { Stylosphaera radiosa } \\
\text { Stylatractus santaeannae } \\
\end{array}$ & R & & & & & ' & & & & & C & $\mathrm{F}$ & $\mathbf{R}$ \\
\hline $\begin{array}{l}\text { Cenosphaera sp. } \\
\text { Siphocampe sp. } \\
\text { Orosphaera sp. ? } \\
\text { Lychnocanoma conica } \\
\text { Syylosphaera sp. B } \\
\end{array}$ & & & & & & & & & & & & & \\
\hline $\begin{array}{l}\text { Lamprocyclas sp. } \\
\text { Carpocanarium papillosum } \\
\text { Cycladophora sp. } \\
\text { Stylosphaera sp. C } \\
\text { Cyrtocapsella tetrapera } \\
\end{array}$ & & $\mathrm{T}$ & $\mathrm{T}$ & $\mathrm{T}$ & & & $\mathrm{T}$ & $\mathrm{R}$ & $\mathrm{T}$ & & & $\mathrm{R}$ & $\mathrm{R}$ \\
\hline $\begin{array}{l}\text { Corythospyris fiscella } \\
\text { Prunopyle hayesi } \\
\text { Amphistylus angelinus } \\
\text { Spongodiscus craticulatus } \\
\text { Stauroxiphos communis }\end{array}$ & $\begin{array}{l}R \\
T \\
T\end{array}$ & $\begin{array}{l}R \\
R \cdot F\end{array}$ & $\stackrel{R}{R \cdot C}$ & $\begin{array}{l}\mathrm{R} \\
\mathrm{T} \\
\mathrm{T}\end{array}$ & $\begin{array}{l}\mathrm{T} \\
\mathrm{T}\end{array}$ & $\mathrm{R}$ & & $\mathrm{T}$ & & & $\mathbf{R}$ & $\mathbf{R}$ & $\mathbf{R}$ \\
\hline $\begin{array}{l}\text { Dendrospyris stabilis } \\
\text { Stylosphaera neptunus } \\
\text { Acrosphaera spp. } \\
\text { Prunopyle sp. B } \\
\text { Ceratocyrtis stigi }\end{array}$ & $\mathbf{R}$ & $\mathrm{T}$ & $\mathrm{R}$ & $\mathbf{R}$ & & & & $\mathbf{R}$ & . & & $\mathrm{R}$ & $\mathbf{R}$ & $\mathrm{T}$ \\
\hline $\begin{array}{l}\text { Eucyrtidium sp. B } \\
\text { Eucyrtidium punctatum } \\
\text { Stylosphaera sp. A } \\
\text { Cycladophora golli regipileus } \\
\text { Cynocapsella longithorax } \\
\end{array}$ & & & & & & & & & & & & $\mathrm{R}$ & $\mathbf{R}$ \\
\hline $\begin{array}{l}\text { Eucyrtidium cienkowskii gr. } \\
\text { Pterocanium sp. } \\
\text { Lamprocyrtis (?) cf. hannai } \\
\text { Gondwanaria deflandrei } \\
\text { Hexacontium cf. enthacanthum }\end{array}$ & $\begin{array}{l}\mathrm{R} \\
\mathrm{R}\end{array}$ & $\begin{array}{c}R \\
R-F\end{array}$ & $\begin{array}{l}\mathrm{R} \\
\mathrm{R}\end{array}$ & $\mathrm{R}$ & $\mathrm{T}$ & $\begin{array}{l}\mathrm{R} \\
\mathrm{R}\end{array}$ & $\begin{array}{l}\mathrm{R} \\
\mathrm{R}\end{array}$ & $\begin{array}{l}\mathrm{F} \\
\mathrm{R} \\
\mathrm{T} \\
\mathrm{F}\end{array}$ & $\begin{array}{l}\mathrm{R} \\
\mathrm{T} \\
\mathrm{R}\end{array}$ & $\mathrm{T}$ & $\mathrm{R}$ & $\mathrm{R}$ & $\begin{array}{l}\mathrm{T} \\
\mathrm{R}\end{array}$ \\
\hline $\begin{array}{l}\text { Druppatractus hastatus } \\
\text { Prmopyle } \mathrm{sp} . \mathrm{D} \\
\text { Lychnocanoma } \mathrm{sp} . \mathrm{B} \\
\text { Cycladophora humerus } \\
\text { Eucyrtidium } \mathrm{sp} . \mathrm{A}\end{array}$ & R-F & $\begin{array}{l}T \\
R \\
F\end{array}$ & $\begin{array}{l}\mathrm{T} \\
\mathrm{T} \\
\mathrm{F}\end{array}$ & $\mathbf{R}$ & $\mathrm{R}$ & $\mathrm{R}$ & $\mathbf{R}$ & $\begin{array}{l}\mathrm{T} \\
\mathrm{R}\end{array}$ & $\mathrm{R}$ & $\mathrm{T}$ & & $\mathrm{T}$ & \\
\hline $\begin{array}{l}\text { Heliodiscus sp. A } \\
\text { Velicucullus cf. oddgumeri } \\
\text { Spongomelissa dilli } \\
\text { Peripyramis circumtexa } \\
\text { Dendrospyris rhodospyroides }\end{array}$ & $\mathrm{R}$ & $\mathrm{R}$ & $\mathrm{R}$ & $\mathrm{R}$ & $\mathrm{T}$ & $\mathrm{R}$ & $\mathbf{R}$ & $\mathrm{F}$ & $\mathrm{R}$ & $\mathrm{T}$ & $\mathrm{R}$ & $\mathrm{R}$ & \\
\hline $\begin{array}{l}\text { Stylodictya validispina } \\
\text { Spongopyle asculosa } \\
\text { Trissocyclid sp. A } \\
\text { Actinomma golownini } \\
\text { Eucyrtidium calvertense }\end{array}$ & $\begin{array}{l}\mathrm{T} \\
\mathrm{R}\end{array}$ & $\begin{array}{l}\mathrm{R} \\
\mathrm{R}\end{array}$ & $\begin{array}{l}\mathrm{R} \\
\mathrm{F}\end{array}$ & $\mathrm{F}$ & $\mathbf{R}$ & $\begin{array}{l}\mathrm{T} \\
\mathrm{R}\end{array}$ & $\begin{array}{l}\mathrm{R} \\
\mathrm{R} \\
\mathrm{T} \\
\mathrm{T} \\
\mathrm{R}\end{array}$ & $\begin{array}{l}\mathrm{R} \\
\mathrm{R} \\
\mathrm{R} \\
\mathrm{R} \\
\mathrm{F}\end{array}$ & $\begin{array}{l}\mathrm{T} \\
\mathrm{T} \\
\mathrm{R} \\
\mathrm{T} \\
\mathrm{T}\end{array}$ & T & $\begin{array}{l}T \\
T \\
T \\
R\end{array}$ & $\begin{array}{l}\mathrm{R} \\
\mathrm{F}\end{array}$ & $\begin{array}{l}\mathrm{F} \\
\mathrm{F}\end{array}$ \\
\hline $\begin{array}{l}\text { Cormutella profunda } \\
\text { Comatella clathrata } \\
\text { Cyrtocapsella cormuta } \\
\text { Antarctissa defandrei } \\
\text { Rhopalodictyum sp. }\end{array}$ & & $\begin{array}{l}\mathbf{R} \\
\mathrm{T}\end{array}$ & $\begin{array}{l}R \\
T \\
R\end{array}$ & & & $\mathrm{~F}$ & $\begin{array}{l}\mathrm{T} \\
\mathrm{F}\end{array}$ & $\mathrm{R}$ & $\mathrm{F}$ & & & $\mathrm{R}$ & \\
\hline $\begin{array}{l}\text { Collosphaerid sp. B } \\
\text { Lithocarpium polyacantha } \\
\text { Cycladophora spongothorax }\end{array}$ & & . & & & & & & $\begin{array}{l}\mathrm{R} \\
\mathrm{R}\end{array}$ & $\begin{array}{l}\mathrm{T} \\
\mathrm{T}\end{array}$ & & & $\mathrm{R}$ & $\begin{array}{l}R \\
R\end{array}$ \\
\hline
\end{tabular}


Table 3. Stratigraphic occurrences of radiolarian species in the early to middle Miocene, Hole 748B.

\begin{tabular}{|c|c|c|c|c|c|c|c|c|c|c|c|c|c|c|c|c|c|c|c|c|c|c|c|}
\hline \multirow{2}{*}{$\begin{array}{l}\text { Zones } \\
\text { Core, section } \\
\text { Interval (cm) }\end{array}$} & \multicolumn{5}{|c|}{ Stylosphaera radiosa } & \multirow{2}{*}{\begin{tabular}{|c|}
$\begin{array}{c}C . \\
\text { antiqua }\end{array}$ \\
$8 \mathrm{H}-2$ \\
$45-47$ \\
\end{tabular}} & \multicolumn{15}{|c|}{ Cycladophora golli regipileus } & \multirow{2}{*}{\begin{tabular}{|c|}
$\begin{array}{c}E . \\
\text { punc- } \\
\text { tatum }\end{array}$ \\
$6 \mathrm{H}-1$ \\
$65-67$ \\
\end{tabular}} & \multirow{2}{*}{$\begin{array}{c}\begin{array}{c}\text { C. } \\
\text { spongo- } \\
\text { thorax }\end{array} \\
\begin{array}{l}5 \mathrm{H}-5 \\
72-76\end{array} \\
\end{array}$} \\
\hline & $\begin{array}{l}8 \mathrm{H}- \\
\mathrm{CC}\end{array}$ & $\begin{array}{r}8 \mathrm{H}-6 \\
45-47 \\
\end{array}$ & $\begin{array}{c}8 \mathrm{H}-5 \\
45-47\end{array}$ & $\begin{array}{c}8 \mathrm{H}-4 \\
45-47 \\
\end{array}$ & $\begin{array}{c}8 \mathrm{H}-3 \\
45-47\end{array}$ & & $\begin{array}{c}8 \mathrm{H}-1 \\
45-47 \\
\end{array}$ & $\begin{array}{l}7 \mathrm{H}- \\
\mathrm{CC}\end{array}$ & $\begin{array}{r}7 \mathrm{H}-6 \\
45-47 \\
\end{array}$ & $\begin{array}{c}7 \mathrm{H}-5 \\
45-47\end{array}$ & $\begin{array}{c}7 \mathrm{H}-4 \\
45-47\end{array}$ & $\begin{array}{c}7 \mathrm{H}-3 \\
45-47 \\
\end{array}$ & $\begin{array}{c}7 \mathrm{H}-2 \\
45-47\end{array}$ & $\begin{array}{c}7 \mathrm{H}-1 \\
45-47 \\
\end{array}$ & $\begin{array}{l}6 \mathrm{H}- \\
\mathrm{CC}\end{array}$ & $\begin{array}{c}6 \mathrm{H}-7 \\
45-47 \\
\end{array}$ & $\begin{array}{r}6 \mathrm{H}-6 \\
45-47 \\
\end{array}$ & $\begin{array}{l}6 \mathrm{H}-5 \\
45-47 \\
\end{array}$ & $\begin{array}{r}6 \mathrm{H}-4 \\
45-47 \\
\end{array}$ & $\begin{array}{c}6 \mathrm{H}-3 \\
45-47\end{array}$ & $\begin{array}{c}6 \mathrm{H}-2 \\
45-47 \\
\end{array}$ & & \\
\hline $\begin{array}{l}\text { Abundance } \\
\text { Preservation }\end{array}$ & $\begin{array}{l}\mathrm{A} \\
\mathrm{G} \\
\end{array}$ & $\begin{array}{l}\mathrm{A} \\
\mathrm{G} \\
\end{array}$ & $\begin{array}{l}\mathrm{C} \\
\mathrm{G} \\
\end{array}$ & $\begin{array}{l}\mathrm{C} \\
\mathrm{G} \\
\end{array}$ & $\begin{array}{l}\text { C } \\
\text { G }\end{array}$ & $\begin{array}{l}\mathrm{A} \\
\mathrm{G}\end{array}$ & $\begin{array}{l}\mathrm{F} \\
\mathrm{M}\end{array}$ & $\begin{array}{l}\mathrm{C} \\
\mathrm{G} \\
\end{array}$ & $\begin{array}{l}\mathrm{A} \\
\mathrm{G}\end{array}$ & $\begin{array}{l}\mathrm{F} \\
\mathrm{M}\end{array}$ & $\begin{array}{l}\mathrm{A} \\
\mathrm{G}\end{array}$ & $\begin{array}{l}\text { F-C } \\
M-G\end{array}$ & $\begin{array}{l}\mathrm{C} \\
\mathrm{G} \\
\end{array}$ & $\begin{array}{l}\mathrm{C} \\
\mathrm{G} \\
\end{array}$ & $\begin{array}{l}\mathrm{C} \\
\mathrm{G} \\
\end{array}$ & $\begin{array}{l}\mathrm{C} \\
\mathrm{G} \\
\end{array}$ & $\begin{array}{l}\mathbf{C} \\
\mathbf{G} \\
\end{array}$ & $\begin{array}{c}\text { R-F } \\
\text { G }\end{array}$ & $\begin{array}{l}\mathrm{F} \\
\mathrm{G}\end{array}$ & $\begin{array}{c}\text { R-F } \\
\text { M }\end{array}$ & $\begin{array}{l}\mathrm{A} \\
\mathrm{G}\end{array}$ & $\begin{array}{l}\mathrm{C} \\
\mathrm{G}\end{array}$ & $\begin{array}{l}\mathrm{C} \\
\mathrm{G}\end{array}$ \\
\hline $\begin{array}{l}\text { Actinomma holtedahli } \\
\text { Actinomma sp. B } \\
\text { Actinomma medusa } \\
\text { Amphistylus angelinus } \\
\text { Stylosphaera radiosa }\end{array}$ & $\begin{array}{l}\mathrm{R} \\
\mathrm{C} \\
\mathrm{F} \\
\mathrm{R} \\
\mathrm{R}\end{array}$ & $\begin{array}{l}\mathrm{R} \\
\mathrm{A} \\
\mathrm{T} \\
\mathrm{R} \\
\mathrm{R}\end{array}$ & $\begin{array}{l}\mathrm{R} \\
\mathrm{C} \\
\mathrm{T} \\
\mathrm{T}\end{array}$ & $\begin{array}{l}\mathrm{C} \\
\mathrm{R} \\
\mathrm{R}\end{array}$ & $\begin{array}{c}R \\
T \\
R \cdot F\end{array}$ & $\begin{array}{l}A \\
R\end{array}$ & $\begin{array}{l}\mathrm{T} \\
\mathrm{R}\end{array}$ & $\begin{array}{l}\mathrm{F} \\
\mathrm{R}\end{array}$ & $\begin{array}{l}\mathrm{C} \\
\mathrm{R}\end{array}$ & $\begin{array}{l}\mathrm{R} \\
\mathrm{R}\end{array}$ & $\begin{array}{l}F \\
R\end{array}$ & $\begin{array}{c}T-R \\
R\end{array}$ & $\begin{array}{l}\mathrm{F} \\
\mathrm{R}\end{array}$ & $\begin{array}{l}\mathbf{R} \\
\mathrm{R}\end{array}$ & $\begin{array}{l}F \\
R\end{array}$ & $\begin{array}{l}\mathrm{F} \\
\mathrm{R}\end{array}$ & $\begin{array}{l}\mathrm{R} \\
\mathrm{R}\end{array}$ & $\begin{array}{l}R \\
R\end{array}$ & $\begin{array}{l}\mathrm{T} \\
\mathrm{R}\end{array}$ & $\begin{array}{l}\mathrm{T} \\
\mathbf{R}\end{array}$ & $\begin{array}{l}\mathbf{R} \\
\mathbf{R}\end{array}$ & $\begin{array}{l}\mathbf{R} \\
\mathbf{R}\end{array}$ & \\
\hline $\begin{array}{l}\text { S. coronata laevis } \\
\text { Cyrtocapsella robusta } \\
\text { Prunopyle hayesi } \\
\text { Prunopyle sp. B } \\
\text { Ceratocyrtis stigi }\end{array}$ & $\begin{array}{l}\mathbf{R} \\
\mathbf{F} \\
\mathbf{R} \\
\mathrm{R} \\
\mathrm{F}\end{array}$ & $\begin{array}{l}\mathrm{R} \\
\mathrm{C} \\
\mathrm{C} \\
\mathrm{F} \\
\mathrm{R}\end{array}$ & $\begin{array}{l}\mathrm{R} \\
\mathrm{T} \\
\mathrm{R} \\
\mathrm{R} \\
\mathrm{R}\end{array}$ & $\begin{array}{l}\mathbf{R} \\
\mathbf{R} \\
\mathbf{R} \\
\mathbf{R} \\
\mathbf{R}\end{array}$ & $\begin{array}{l}\mathrm{R} \\
\mathrm{R} \\
\mathrm{R} \\
\mathrm{R}\end{array}$ & $\begin{array}{l}\mathrm{R} \\
\mathrm{R} \\
\mathrm{R}\end{array}$ & $\begin{array}{l}\mathrm{R} \\
\mathrm{R} \\
\mathrm{R}\end{array}$ & $\begin{array}{l}\mathrm{R} \\
\mathrm{R} \\
\mathrm{R}\end{array}$ & $\begin{array}{l}\mathbf{R} \\
\mathbf{R} \\
\mathbf{R}\end{array}$ & $\begin{array}{l}\mathbf{R} \\
\mathbf{R} \\
\mathbf{R}\end{array}$ & $\begin{array}{l}\mathrm{R} \\
\mathrm{R} \\
\mathrm{R}\end{array}$ & $\begin{array}{l}\mathbf{R} \\
\mathbf{R} \\
\mathbf{R}\end{array}$ & $\begin{array}{l}\mathbf{R} \\
\mathbf{R} \\
\mathbf{R}\end{array}$ & $\begin{array}{l}\mathbf{R} \\
\mathbf{R} \\
\mathbf{R}\end{array}$ & $\begin{array}{l}\mathrm{F} \\
\mathrm{R} \\
\mathrm{R}\end{array}$ & $\begin{array}{l}\mathrm{F} \\
\mathrm{R} \\
\mathrm{R}\end{array}$ & $\begin{array}{l}\mathbf{R} \\
\mathbf{R} \\
\mathbf{R}\end{array}$ & $\begin{array}{l}\mathbf{R} \\
\mathbf{R} \\
\mathbf{R}\end{array}$ & $\begin{array}{l}\mathbf{R} \\
\mathbf{R} \\
\mathbf{R}\end{array}$ & $\begin{array}{l}T \\
\text { R } \\
\mathbf{R}\end{array}$ & $\begin{array}{l}\mathbf{R} \\
\mathbf{R} \\
\mathbf{R}\end{array}$ & $\begin{array}{l}\mathbf{R} \\
\mathbf{R} \\
\mathbf{R}\end{array}$ & $\begin{array}{l}\mathbf{R} \\
\mathrm{R} \\
\mathrm{T}\end{array}$ \\
\hline $\begin{array}{l}\text { Carpocanarium papillosum } \\
\text { Velicucullus altus } \\
\text { Lychnocanoma conica } \\
\text { Spongodiscus craticulatus } \\
\text { Lithomelissa robusta }\end{array}$ & $\begin{array}{l}\mathrm{R} \\
\mathrm{T} \\
\mathrm{F} \\
\mathrm{T} \\
\mathrm{R}-\mathrm{F}\end{array}$ & $\begin{array}{l}\mathbf{R} \\
\mathbf{R} \\
\mathrm{R} \\
\mathrm{R}\end{array}$ & $\begin{array}{l}\mathrm{T} \\
\mathrm{T} \\
\mathrm{F} \\
\mathrm{R}\end{array}$ & $\begin{array}{l}\mathrm{R} \\
\mathrm{R} \\
\mathrm{F} \\
\mathrm{T}\end{array}$ & $\begin{array}{l}\mathrm{R} \\
\mathrm{R} \\
\mathrm{R}\end{array}$ & $\begin{array}{l}\mathrm{R} \\
\mathrm{R} \\
\mathrm{F}\end{array}$ & $\mathrm{R}$ & $\begin{array}{l}\mathrm{R} \\
\mathrm{T}\end{array}$ & $\begin{array}{l}\mathrm{R} \\
\mathrm{F}\end{array}$ & $\mathbf{R}$ & $\begin{array}{l}\mathbf{R} \\
\mathrm{R}\end{array}$ & $\mathrm{R}$ & $\bar{R}$ & $\mathbf{R}$ & $\mathrm{R}$ & $\mathrm{R}$ & $\mathbf{R}$ & $\mathbf{R}$ & $\begin{array}{l}\mathbf{R} \\
\mathbf{F}\end{array}$ & $\begin{array}{l}\mathbf{R} \\
\mathrm{T}\end{array}$ & $\begin{array}{l}\mathrm{R} \\
\mathrm{R}\end{array}$ & $\begin{array}{l}\mathrm{R} \\
\mathrm{F}\end{array}$ & $\mathbf{R}$ \\
\hline $\begin{array}{l}\text { Spongopyle osculosa } \\
\text { Lamprocyclas sp. } \\
\text { Spongotrochus sp. } \\
\text { Stylosphaera sp. B } \\
\text { Tholoniid sp. B } \\
\end{array}$ & $\begin{array}{l}\mathrm{R} \\
\mathrm{T} \\
\mathrm{R} \\
\mathrm{R}\end{array}$ & $\begin{array}{l}\mathrm{R} \\
\mathrm{T} \\
\mathrm{R}\end{array}$ & $\mathbf{R}$ & $\begin{array}{l}\mathrm{R} \\
\mathrm{F}\end{array}$ & & $\begin{array}{l}T \\
\mathbf{R}\end{array}$ & & $\mathrm{R}$ & $\begin{array}{l}R \\
R\end{array}$ & & $\begin{array}{l}\mathrm{R} \\
\mathrm{R}\end{array}$ & $T$ & $\begin{array}{l}\mathrm{R} \\
\mathrm{R}\end{array}$ & 1 & $\begin{array}{l}\mathbf{R} \\
\mathrm{R} \\
\mathrm{T}\end{array}$ & $\begin{array}{l}\mathrm{R} \\
\mathrm{R}\end{array}$ & $\begin{array}{l}\mathrm{R} \\
\mathrm{R}\end{array}$ & $\begin{array}{l}\mathrm{T} \\
\mathrm{R} \\
\mathrm{R}\end{array}$ & $\begin{array}{l}\mathrm{T} \\
\mathrm{T} \\
\mathrm{T}\end{array}$ & $T$ & $\begin{array}{l}\mathrm{T} \\
\mathrm{R}\end{array}$ & $\begin{array}{l}\mathbf{R} \\
\mathbf{R} \\
\mathrm{T}\end{array}$ & \\
\hline $\begin{array}{l}\text { Cenosphaera sp. A } \\
\text { Velicucullus cf. oddgurneri } \\
\text { Artophormis gracilis } \\
\text { Gondwanaria japonica } \\
\text { Prunopyle sp. A }\end{array}$ & & $\begin{array}{l}\mathrm{R}-\mathrm{F} \\
\mathrm{R} \\
\mathrm{T} \\
\mathrm{R} \\
\mathrm{R} \\
\end{array}$ & $\begin{array}{l}\mathrm{R} \\
\mathrm{T} \\
\mathrm{R}\end{array}$ & $\begin{array}{l}\mathrm{R} \\
\mathrm{R} \\
\mathrm{F}\end{array}$ & $\begin{array}{l}\mathbf{R} \\
\mathbf{R}\end{array}$ & $\begin{array}{l}\mathrm{T} \\
\mathrm{F} \\
\mathrm{R}\end{array}$ & $\begin{array}{l}\mathrm{R} \\
\mathrm{R}\end{array}$ & $\begin{array}{l}\mathrm{T} \\
\mathrm{R} \\
\mathrm{R}\end{array}$ & $\begin{array}{l}\mathbf{R} \\
\mathbf{R}\end{array}$ & $\begin{array}{l}\mathbf{R} \\
\mathbf{R}\end{array}$ & R & $\begin{array}{l}\mathbf{R} \\
\mathrm{R} \\
\mathrm{R}\end{array}$ & $\mathbf{R}$ & $\begin{array}{l}\mathbf{R} \\
\mathbf{R}\end{array}$ & $\mathbf{R}$ & $\mathbf{R}$ & R & $\mathbf{R}$ & R & $\mathbf{R}$ & $\mathbf{R}$ & $\mathbf{R}$ & $\mathbf{R}$ \\
\hline $\begin{array}{l}\text { Ceratocyrtis mashae } \\
\text { Corythospyris fiscella } \\
\text { Eucyrtidium cienkowskii gr. } \\
\text { Prunopyle titan } \\
\text { Cycladophora sp. }\end{array}$ & & $\begin{array}{l}\mathbf{R} \\
\mathbf{T}\end{array}$ & $\begin{array}{l}\mathrm{R} \\
\mathrm{T}\end{array}$ & $\begin{array}{l}\mathrm{R} \\
\mathrm{R} \\
\mathrm{T} \\
\mathrm{R}\end{array}$ & $\begin{array}{l}\mathrm{R} \\
\mathrm{R} \\
\mathrm{T} \\
\mathrm{R}\end{array}$ & $\begin{array}{l}\mathrm{R} \\
\mathrm{R} \\
\mathrm{F} \\
\mathrm{R}\end{array}$ & $\begin{array}{l}\mathrm{R} \\
\mathrm{R}\end{array}$ & $\begin{array}{l}\mathrm{R} \\
\mathrm{R} \\
\mathrm{R}\end{array}$ & $\begin{array}{l}\mathrm{R} \\
\mathrm{R} \\
\mathrm{T}\end{array}$ & $\begin{array}{l}\mathrm{T} \\
\mathrm{T} \\
T\end{array}$ & $\begin{array}{l}\mathrm{R} \\
\mathrm{T} \\
\mathrm{R} \\
\mathrm{R}\end{array}$ & $\begin{array}{l}\mathrm{T} \\
\mathrm{R} \\
\mathrm{T}\end{array}$ & $\begin{array}{l}\mathrm{R} \\
\mathrm{T} \\
\mathrm{F} \\
\mathrm{R}\end{array}$ & $\begin{array}{l}\mathrm{R} \\
\mathrm{R} \\
\mathrm{R}\end{array}$ & $\begin{array}{l}\mathrm{R} \\
\mathrm{T} \\
\mathrm{R} \\
\mathrm{T}\end{array}$ & $\begin{array}{l}\mathbf{R} \\
\mathbf{R} \\
\mathbf{R}\end{array}$ & $\begin{array}{l}\mathrm{T} \\
\mathrm{R}\end{array}$ & $\begin{array}{l}\mathrm{T} \\
\mathbf{R} \\
\mathrm{R}\end{array}$ & $\begin{array}{l}\mathrm{R} \\
\mathrm{R}\end{array}$ & $\mathbf{T}$ & $\begin{array}{l}\mathrm{R} \\
\mathrm{F}\end{array}$ & \begin{tabular}{c|}
$R$ \\
$R-F$
\end{tabular} & $\begin{array}{c}R \\
R \\
R-F\end{array}$ \\
\hline $\begin{array}{l}\text { Lamprocyclas sp. } \\
\text { Cyrtocapsella tetrapera } \\
\text { Cyctampterium milowi } \\
\text { Cycladophora antiqua } \\
\text { Stauroxiphos communis }\end{array}$ & & & & & $\mathrm{T}$ & $\begin{array}{l}\mathrm{R} \\
T \\
\mathrm{R}\end{array}$ & $\mathrm{T}$ & $\begin{array}{l}\mathrm{R} \\
\mathrm{F}\end{array}$ & $\begin{array}{l}R \\
F\end{array}$ & $\begin{array}{l}R \\
T\end{array}$ & $\begin{array}{l}\mathbf{R} \\
\mathbf{R}\end{array}$ & $\begin{array}{l}\mathrm{R} \\
\mathrm{R}\end{array}$ & $\begin{array}{l}\mathbf{R} \\
\mathbf{R} \\
\mathrm{T}\end{array}$ & $\begin{array}{l}\mathbf{R} \\
\mathbf{R}\end{array}$ & $\begin{array}{l}R \\
R\end{array}$ & R & $\begin{array}{c}R \\
R \\
F \cdot C \\
\end{array}$ & R & $\begin{array}{c}\mathrm{T} \\
\mathrm{T}-\mathrm{R}\end{array}$ & $\mathrm{R}$ & R & R & \\
\hline $\begin{array}{l}\text { Stylosphaera sp. C } \\
\text { Dendrospyris stabilis } \\
\text { Cycladophora conica } \\
\text { Cyrtocapsella longithorax } \\
\text { Lithomelissa cf. ehrenbergii }\end{array}$ & & & & & & $\begin{array}{l}\mathrm{R} \\
\mathrm{T} \\
\mathrm{R}\end{array}$ & $\begin{array}{l}\mathrm{R} \\
\mathrm{R} \\
\mathrm{R}\end{array}$ & $\begin{array}{c}\mathrm{T} \\
\mathrm{R} \\
\mathrm{R} \\
\mathrm{F} \\
\mathrm{R}-\mathrm{F}\end{array}$ & $\begin{array}{l}\mathrm{R} \\
\mathrm{R} \\
\mathrm{F}\end{array}$ & $\begin{array}{l}\mathbf{R} \\
\mathrm{R}\end{array}$ & $\begin{array}{l}\mathrm{T} \\
\mathrm{R} \\
\mathrm{F} \\
\mathrm{F}\end{array}$ & $\begin{array}{l}\mathrm{R} \\
\mathrm{R} \\
\mathrm{R} \\
\mathrm{R}\end{array}$ & $\begin{array}{l}\mathbf{R} \\
\mathbf{R} \\
\mathbf{R} \\
\mathbf{R}\end{array}$ & $\begin{array}{l}\mathbf{R} \\
\mathbf{R} \\
\mathbf{R} \\
\mathbf{R}\end{array}$ & $\begin{array}{l}\mathrm{R} \\
\mathrm{R} \\
\mathrm{F} \\
\mathrm{R}\end{array}$ & $\begin{array}{l}\mathrm{T} \\
\mathrm{R} \\
\mathrm{R} \\
\mathrm{T}\end{array}$ & $\begin{array}{l}\mathbf{R} \\
\mathbf{R} \\
\mathrm{T}\end{array}$ & $\begin{array}{l}\mathbf{R} \\
\mathbf{R}\end{array}$ & $\mathbf{R}$ & $\mathbf{R}$ & $\mathbf{R}$ & $\begin{array}{l}\mathrm{R} \\
\mathrm{T} \\
\mathrm{T}\end{array}$ & $\mathbf{R}$ \\
\hline $\begin{array}{l}\text { Cycladophora golli regipileus } \\
\text { Cycladophora golli golli } \\
\text { Gondwanaria defiandrei } \\
\text { Eucyrtidium calvertense gr. } \\
\text { Rhopalodictyum sp. } \\
\end{array}$ & & & & & & & $\begin{array}{l}\mathrm{T} \\
\mathrm{R}\end{array}$ & $\begin{array}{l}\mathbf{R} \\
\mathbf{R} \\
\mathbf{R}\end{array}$ & $\begin{array}{l}\mathrm{R} \\
\mathrm{R} \\
\mathrm{R}\end{array}$ & $\begin{array}{l}\mathrm{R} \\
\mathrm{R} \\
\mathrm{T}\end{array}$ & $\begin{array}{l}\mathrm{R} \\
\mathrm{R} \\
\mathrm{R} \\
\mathrm{R}\end{array}$ & $\begin{array}{l}\mathrm{T} \\
\mathrm{R} \\
\mathrm{R}\end{array}$ & $\begin{array}{l}\mathrm{R} \\
\mathrm{F} \\
\mathrm{R} \\
\mathrm{R} \\
\mathrm{T}\end{array}$ & $\begin{array}{l}\mathrm{R} \\
\mathrm{F} \\
\mathrm{R} \\
\mathrm{R}\end{array}$ & $\begin{array}{l}\mathrm{F} \\
\mathrm{R} \\
\mathrm{R}\end{array}$ & $\begin{array}{l}\mathrm{F} \\
\mathrm{R}\end{array}$ & $\begin{array}{c}\text { F-C } \\
\text { T }\end{array}$ & $\begin{array}{l}\mathrm{F} \\
\mathrm{R} \\
\mathrm{T}\end{array}$ & $\begin{array}{l}\mathrm{R} \\
\mathrm{R}\end{array}$ & $\begin{array}{l}\mathrm{R} \\
\mathrm{T}\end{array}$ & $\begin{array}{l}\mathrm{C} \\
\mathrm{T} \\
\mathrm{T}\end{array}$ & $\begin{array}{l}\mathrm{C} \\
\mathrm{R} \\
\mathrm{T}\end{array}$ & $\mathbf{R}$ \\
\hline $\begin{array}{l}\text { Acrosphaera spp. } \\
\text { Circodiscus ellipticus } \\
\text { Trissocyclid sp. A } \\
\text { Dendrospyris hodospyroides } \\
\text { Gondwanaria sp. A }\end{array}$ & & & & $*$ & & & $\mathrm{~T}$ & 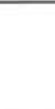 & 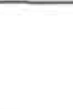 & $\begin{array}{l}\mathrm{T} \\
\mathrm{T}\end{array}$ & & & $\begin{array}{l}\mathrm{R} \\
\mathrm{R}\end{array}$ & $\mathrm{T}$ & $\begin{array}{l}\mathrm{R} \\
\mathrm{R} \\
\mathrm{R} \\
\mathrm{T}\end{array}$ & $\begin{array}{l}\mathbf{R} \\
\mathbf{R} \\
\mathbf{R} \\
\mathbf{R} \\
\mathrm{T}\end{array}$ & $\begin{array}{l}\mathbf{R} \\
\mathbf{F} \\
\mathbf{R}\end{array}$ & $\underset{\mathrm{R}-\mathrm{F}}{\mathrm{T}}$ & $\begin{array}{l}\mathbf{R} \\
\mathrm{T} \\
\mathrm{T} \\
\mathbf{R}\end{array}$ & $\begin{array}{l}T \\
R\end{array}$ & $\begin{array}{l}\mathrm{R} \\
\mathrm{T} \\
\mathrm{T} \\
\mathrm{R} \\
\mathrm{T}\end{array}$ & $\begin{array}{l}\mathrm{T} \\
\mathrm{T} \\
\mathrm{T} \\
\mathrm{R}\end{array}$ & $\begin{array}{l}\mathrm{R} \\
\mathrm{R} \\
\mathrm{R}\end{array}$ \\
\hline $\begin{array}{l}\text { Lychnocanoma sp. C } \\
\text { Actinomma sp. A } \\
\text { Stylatractus neptumus } \\
\text { Stylosphaera sp. A } \\
\text { Hexacontium cf. enthacanthum }\end{array}$ & & & & & & & & & & & & & & & & $\begin{array}{l}\mathrm{T} \\
\mathrm{T}\end{array}$ & $\begin{array}{l}\mathrm{T} \\
\mathrm{T}\end{array}$ & $\begin{array}{l}\mathrm{T} \\
\mathrm{R} \\
\mathrm{T}\end{array}$ & $\begin{array}{l}\mathrm{T} \\
\mathrm{T}\end{array}$ & $\begin{array}{l}\mathrm{T} \\
\mathrm{T}\end{array}$ & $\begin{array}{l}\mathbf{R} \\
\mathbf{R} \\
\mathrm{T}\end{array}$ & $\begin{array}{l}\mathrm{T} \\
\mathbf{R} \\
\mathbf{R}\end{array}$ & $\begin{array}{l}\mathrm{R} \\
\mathrm{R}\end{array}$ \\
\hline $\begin{array}{l}\text { Stichophormis sp. } \\
\text { Prunopyle tetrapila } \\
\text { Eucyrtidium punctatum } \\
\text { Anthocyrtidium sp. } \\
\text { Cycladophora humerus }\end{array}$ & & & & & & & & & & & & & & & & & & & $\mathrm{T}$ & & $\mathrm{T}$ & $\begin{array}{l}\mathrm{T} \\
\mathrm{T} \\
\mathrm{F} \\
\mathrm{T}\end{array}$ & R \\
\hline $\begin{array}{l}\text { Actinomma golownini } \\
\text { Cycladophora spongothorax }\end{array}$ & & & & & & & & & & & & & & & & & & & & & & & $\begin{array}{l}\mathrm{F} \\
\mathrm{R}\end{array}$ \\
\hline
\end{tabular}

Note. Abundance: $\mathrm{A}=$ abundant, $\mathrm{C}=$ common, $\mathrm{F}=$ few, $\mathrm{R}=$ rare, and $\mathrm{T}=$ trace. Preservation: $\mathrm{G}=$ good, $\mathrm{M}=\mathrm{moderate}$, and $\mathrm{P}=$ poor.

4, $98-100 \mathrm{~cm}$, and $-18 \mathrm{H}-2,98-100 \mathrm{~cm}$. At the same level, Actinomma sp. B, Stauroxiphos communis, Dictyophimus gracilipes, Velicucullus cf. oddgurneri, Gondwanaria sp. A, and Cycladophora golli golli occur for the first time. One can speculate that the first occurrences and species abundance patterns indicate the presence of a disconformity. However, other stratigraphic or lithologic data supporting this suggestion are not available.

The boundary of the $C$. golli regipileus and Eucyrtidium punctatum zones, which is marked by the FO of E. punctatum, is recognized between Sample 120-751A-16H-1, 98-100 $\mathrm{cm}$, and Section 120-751A-15H-CC. Characteristic radiolarian taxa in the Eucyrtidium punctatum Zone are Stauroxiphos communis, Stichophormis sp., Thyrsocyrtis clausa, Lamprocyrtis(?) cf. hannai, and Cyrtocapsella tetrapera, the latter species occurring commonly.
Above the $E$. punctatum Zone follows the $C$. humerus Zone, recognized between Samples 120-751A-13H-3, 98-100 $\mathrm{cm}$, and $-13 \mathrm{H}-3,32-36 \mathrm{~cm}(113.2-112.6 \mathrm{mbsf})$, because of the first consistent occurrence of its nominate species within this interval. In Hole 751A this zone, which has its zonal base at ca. 14.2 Ma, only occurs during a short interval because most of the zone is omitted by a hiatus, placed between Sample 120-751A-13H-1, 63-69 cm, and Section 120-751A-12H-CC (109.7-109.2 mbsf). The hiatus also encompasses the lower Actinomma golownini Subzone and is interpreted to span a time interval from $\sim 14.0$ to $\sim 12.5 \mathrm{Ma}$, therefore. This can be deduced from the occurrence of $D$. megalocephalis, the marker species of the upper A. golownini Subzone, above the hiatus. This hiatus is also marked by a drastic alteration of the sedimentary facies, from a nannofossil diatom ooze to a nannofossil ooze with diatoms (Schlich, Wise, et al., 1989). 


\section{A. ABELMANN}

Table 4. Stratigraphic occurrences of radiolarian species in the early to middle Mocene, Hole $751 \mathrm{~A}$.

\begin{tabular}{|c|c|c|c|c|c|c|c|c|c|c|c|c|c|c|c|c|c|c|}
\hline \multirow{2}{*}{$\begin{array}{l}\text { Zones } \\
\text { Core, section } \\
\text { Interval }(\mathrm{cm})\end{array}$} & \multicolumn{4}{|c|}{ C. longithorax } & \multicolumn{14}{|c|}{ Cycladophora golli regipileus } \\
\hline & $\begin{array}{l}18 \mathrm{H}- \\
\mathrm{CC}\end{array}$ & $\begin{array}{l}18 \mathrm{H}-6 \\
98-100\end{array}$ & $\begin{array}{l}18 \mathrm{H}-5 \\
98-100\end{array}$ & $\begin{array}{r}18 \mathrm{H}-4 \\
98-100\end{array}$ & $\begin{array}{c}18 \mathrm{H}-2 \\
98-100\end{array}$ & \begin{tabular}{|c|}
$18 \mathrm{H}-1$ \\
$98-100$
\end{tabular} & $\begin{array}{l}17 \mathrm{H}-6 \\
98-100\end{array}$ & $\begin{array}{l}17 \mathrm{H}-5 \\
98-100\end{array}$ & $\begin{array}{r}17 \mathrm{H}-4 \\
98-100\end{array}$ & $\begin{array}{c}17 \mathrm{H}-3 \\
98-100\end{array}$ & $\begin{array}{r}17 \mathrm{H}-2 \\
98-100\end{array}$ & $\begin{array}{c}17 \mathrm{H}-1 \\
98-100\end{array}$ & $\begin{array}{c}16 \mathrm{H}- \\
\mathrm{CC}\end{array}$ & $\begin{array}{r}16 \mathrm{H}-6 \\
98-100\end{array}$ & $\begin{array}{r}16 \mathrm{H}-5 \\
98-100\end{array}$ & $\begin{array}{r}16 \mathrm{H}-4 \\
98-100\end{array}$ & $\begin{array}{c}16 \mathrm{H}-3 \\
98-100\end{array}$ & $\begin{array}{c}16 \mathrm{H}-1 \\
98-100\end{array}$ \\
\hline $\begin{array}{l}\text { Abundance } \\
\text { Preservation }\end{array}$ & $\begin{array}{c}\mathrm{C}-\mathrm{A} \\
\mathrm{G}\end{array}$ & $\begin{array}{l}\mathrm{F} \\
\mathrm{G}\end{array}$ & $\underset{M-G}{F}$ & $\begin{array}{l}\mathrm{F} \\
\mathrm{G}\end{array}$ & $\begin{array}{l}F \\
G\end{array}$ & $\begin{array}{l}F \\
G\end{array}$ & $\begin{array}{l}\mathrm{C} \\
\mathrm{G}\end{array}$ & $\begin{array}{l}F \\
G\end{array}$ & $\begin{array}{l}\mathrm{F} \\
\mathrm{G}\end{array}$ & $\begin{array}{c}\text { F-C } \\
G\end{array}$ & $\begin{array}{l}\mathrm{C} \\
\mathrm{G}\end{array}$ & $\begin{array}{l}\mathrm{R} \\
\mathrm{M}\end{array}$ & $\begin{array}{l}\mathrm{C} \\
\mathrm{G}\end{array}$ & $\begin{array}{l}\mathrm{C} \\
\mathrm{G}\end{array}$ & $\begin{array}{l}\text { C } \\
\text { G }\end{array}$ & $\begin{array}{l}\text { C } \\
\text { G }\end{array}$ & $\begin{array}{l}\text { C } \\
\text { G }\end{array}$ & $\begin{array}{l}\mathrm{C} \\
\mathrm{G}\end{array}$ \\
\hline $\begin{array}{l}\text { Cyrtocapsella tetrapera } \\
\text { Cyrtocapsella longithorax } \\
\text { Lithomelissa cf. ehrenbergi } \\
\text { Amphistylus angelinus } \\
\text { Cycladophora antiqua }\end{array}$ & $\begin{array}{l}\text { F } \\
\text { R-F } \\
\text { R-F } \\
\text { R } \\
\text { F }\end{array}$ & $\begin{array}{l}\text { R-F } \\
\text { R-F } \\
\text { F } \\
\text { R } \\
\text { R }\end{array}$ & $\begin{array}{c}\mathrm{R} \\
\mathrm{R} \\
\mathrm{R}-\mathrm{F} \\
\mathrm{R} \\
\mathrm{F}\end{array}$ & $\begin{array}{c}R \\
R-F \\
R \\
R \\
R-F\end{array}$ & $\begin{array}{l}\mathrm{R} \\
\mathrm{R} \\
\mathrm{R} \\
\mathrm{R}\end{array}$ & $\begin{array}{l}\mathrm{R} \\
\mathrm{R} \\
\mathrm{R} \\
\mathrm{T}\end{array}$ & $\begin{array}{l}\mathrm{R} \\
\mathrm{R} \\
\mathrm{T} \\
\mathrm{R} \\
\mathrm{R}\end{array}$ & $\begin{array}{c}\mathrm{R} \\
\mathrm{R} \\
\mathrm{T}-\mathrm{R} \\
\mathrm{R} \\
\mathrm{R}\end{array}$ & $\begin{array}{l}\mathrm{R} \\
\mathrm{R} \\
\mathrm{R} \\
\mathrm{R} \\
\mathrm{R}\end{array}$ & $\begin{array}{l}\mathrm{R} \\
\mathrm{R} \\
\mathrm{R} \\
\mathrm{R} \\
\mathrm{R}\end{array}$ & $\begin{array}{l}\mathbf{R} \\
\mathbf{R} \\
\mathbf{R} \\
\mathbf{R}\end{array}$ & $\mathrm{T}$ & $\begin{array}{l}\mathrm{R} \\
\mathrm{R} \\
\mathrm{R}\end{array}$ & $\begin{array}{l}\mathrm{R} \\
\mathrm{R}\end{array}$ & $\mathrm{T}$ & $\mathbf{R}$ & $\begin{array}{l}\mathrm{T} \\
\mathrm{R}\end{array}$ & $\mathbf{R}$ \\
\hline $\begin{array}{l}\text { Prunopyle sp. A } \\
\text { Velicucullus altus } \\
\text { Circodiscus ellipticus } \\
\text { Stylatractus neptunus } \\
\text { Prunopyle sp. D }\end{array}$ & $\begin{array}{c}\text { F } \\
F \\
R-F \\
R-F \\
R\end{array}$ & $\begin{array}{c}\mathrm{T}-\mathrm{R} \\
\mathrm{R} \\
\mathrm{R} \\
\mathrm{R}\end{array}$ & $\begin{array}{l}\mathrm{R} \\
\mathrm{T} \\
\mathrm{R} \\
\mathrm{R}\end{array}$ & $\begin{array}{l}\mathrm{T} \\
\mathrm{T} \\
\mathrm{R} \\
\mathrm{R}\end{array}$ & $\begin{array}{l}\mathrm{T} \\
\mathrm{R} \\
\mathrm{T} \\
\mathrm{R}\end{array}$ & $\begin{array}{l}\mathrm{R} \\
\mathrm{R} \\
\mathrm{R}\end{array}$ & $\begin{array}{c}\text { R-F } \\
\text { R } \\
T\end{array}$ & $\begin{array}{c}\mathrm{R} \cdot \mathrm{F} \\
\mathrm{R} \\
\mathrm{R}\end{array}$ & $\begin{array}{l}\mathrm{R} \\
\mathrm{R} \\
\mathrm{T} \\
\mathrm{R}\end{array}$ & $\begin{array}{l}\mathrm{R} \\
\mathrm{R} \\
\mathrm{T} \\
\mathrm{R}\end{array}$ & & & $\begin{array}{l}\mathrm{F} \\
\mathrm{R} \\
\mathrm{F}\end{array}$ & $\begin{array}{c}\mathrm{R} \\
\mathrm{T}-\mathrm{R} \\
\mathrm{R} \\
\mathrm{R}\end{array}$ & $\begin{array}{l}\mathrm{R} \\
\mathrm{T} \\
\mathrm{R} \\
\mathrm{R}\end{array}$ & $\begin{array}{l}\mathrm{R} \\
\mathrm{T} \\
\mathrm{R} \\
\mathrm{R}\end{array}$ & $\begin{array}{l}\mathrm{R} \\
\mathrm{T} \\
\mathrm{R} \\
\mathrm{R} \\
\mathrm{R}\end{array}$ & $\begin{array}{l}\mathrm{F} \\
\mathrm{R} \\
\mathrm{R} \\
\mathrm{R}\end{array}$ \\
\hline $\begin{array}{l}\text { Hexacontium cf. enthacanthum } \\
\text { Corythospyris fiscella } \\
\text { Tripilidium clavipes } \\
\text { Gondwanaria japonica } \\
\text { Cycladophora conica }\end{array}$ & $\begin{array}{c}\mathrm{T}-\mathrm{R} \\
\mathrm{R} \\
\mathrm{R} \\
\mathrm{R} \\
\mathrm{R}\end{array}$ & $\begin{array}{c}\mathrm{T} \\
\mathrm{R} \\
\mathrm{R}-\mathrm{F} \\
\mathrm{R} \\
\mathrm{R}\end{array}$ & $\begin{array}{l}\mathrm{T} \\
\mathrm{R} \\
\mathrm{R} \\
\mathrm{R} \\
\mathrm{R}\end{array}$ & $\begin{array}{c}\mathrm{T} \\
\mathrm{T}-\mathrm{R} \\
\mathrm{R} \\
\mathrm{R} \\
\mathrm{R}\end{array}$ & $\begin{array}{c}\mathrm{T} \\
\mathrm{R} \\
\mathrm{R} \\
\mathrm{R} \\
\mathrm{R}-\mathrm{F}\end{array}$ & $\begin{array}{l}\mathrm{R} \\
\mathrm{R} \\
\mathrm{R} \\
\mathrm{R}\end{array}$ & $\begin{array}{c}\mathrm{T}-\mathrm{R} \\
\mathrm{R} \\
\mathrm{R} \\
\mathrm{R}\end{array}$ & $\begin{array}{l}\mathrm{T} \\
\mathrm{R} \\
\mathrm{R} \\
\mathrm{R} \\
\mathrm{R}\end{array}$ & $\begin{array}{l}\mathrm{R} \\
\mathrm{R} \\
\mathrm{R} \\
\mathrm{R}\end{array}$ & $\begin{array}{l}\mathrm{R} \\
\mathrm{R} \\
\mathrm{R} \\
\mathrm{R}\end{array}$ & $\begin{array}{l}\mathrm{R} \\
\mathrm{R}\end{array}$ & $\mathrm{R}$ & $\begin{array}{l}\mathrm{R} \\
\mathrm{R}\end{array}$ & $\mathrm{T}$ & $\mathrm{T}$ & $\mathrm{T}$ & $T$ & $\mathrm{~T}$ \\
\hline $\begin{array}{l}\text { Peripyramis circumtexta } \\
\text { Druppatractus hastatus } \\
\text { Ceratocyrtis mashae } \\
\text { Dendrospyris stabilis } \\
\text { Acrosphaera } \text { spp. }\end{array}$ & $\begin{array}{l}\mathrm{R} \\
\mathrm{R} \\
\mathrm{T} \\
\mathrm{T} \\
\mathrm{R}\end{array}$ & $\begin{array}{l}\mathbf{R} \\
\mathbf{R} \\
\mathrm{T} \\
\mathrm{T}\end{array}$ & $\begin{array}{l}\mathbf{R} \\
\mathbf{R} \\
\mathrm{T} \\
\mathbf{R}\end{array}$ & $\begin{array}{c}R \\
R-F \\
T \\
R \\
T\end{array}$ & $\begin{array}{l}\mathrm{R} \\
\mathrm{T} \\
\mathrm{R} \\
\mathrm{T}\end{array}$ & $\mathrm{R}$ & $\begin{array}{l}\mathrm{R} \\
\mathrm{T} \\
\mathrm{R}\end{array}$ & $\begin{array}{l}\mathrm{R} \\
\mathrm{R}\end{array}$ & $\begin{array}{c}R \\
T-R \\
R \\
R\end{array}$ & $\begin{array}{c}\mathbf{R} \\
\mathrm{T}-\mathrm{R} \\
\mathbf{R}\end{array}$ & $\mathrm{R}$ & $\begin{array}{l}\mathrm{R} \\
\mathrm{R}\end{array}$ & $\mathrm{R}$ & $\mathrm{R}$ & $\mathrm{T}$ & $\begin{array}{c}\mathrm{R} \\
\mathrm{T} \cdot \mathrm{R}\end{array}$ & $\begin{array}{c}\mathrm{T}-\mathrm{R} \\
\mathrm{T}\end{array}$ & \\
\hline $\begin{array}{l}\text { Siphocampe arachnea gr. } \\
\text { Antarctissa robusta } \\
\text { Eucyrtidium cienkowskii gr. } \\
\text { Ceratocyrtis stigi } \\
\text { Prunopyle sp. B }\end{array}$ & R & $\begin{array}{l}\mathrm{R} \\
\mathrm{R} \\
\mathrm{R}\end{array}$ & $\begin{array}{l}\mathbf{R} \\
\mathbf{R} \\
\mathbf{R}\end{array}$ & $\begin{array}{c}R \\
R \\
R-F \\
R \\
R\end{array}$ & $\begin{array}{c}R \\
R-F \\
R \\
R \\
R\end{array}$ & $\begin{array}{c}R \\
R-F \\
R \\
R \\
R \\
\end{array}$ & $\begin{array}{c}R \\
R-F \\
R \\
R \\
R \\
\end{array}$ & $\begin{array}{c}R \\
R-F \\
R \\
R \\
R\end{array}$ & $\begin{array}{c}R \\
R-F \\
R \\
R \\
R\end{array}$ & $\begin{array}{c}R \\
R-F \\
R \\
R \\
R\end{array}$ & $\begin{array}{l}R \\
C \\
R \\
F \\
F\end{array}$ & $\begin{array}{l}\mathrm{R} \\
\mathrm{R} \\
\mathrm{R} \\
\mathrm{R}\end{array}$ & $\begin{array}{c}\mathrm{R} \\
\mathrm{F}-\mathrm{C} \\
\mathrm{R} \\
\mathrm{R}\end{array}$ & $\begin{array}{c}\mathrm{F} \\
\mathrm{R}-\mathrm{F} \\
\mathrm{R}-\mathrm{F} \\
\mathrm{R}\end{array}$ & $\begin{array}{l}\mathrm{T} \\
\mathrm{F} \\
\mathrm{R} \\
\mathrm{R}\end{array}$ & $\begin{array}{l}\mathrm{R} \\
\mathrm{F} \\
\mathrm{R}\end{array}$ & $\begin{array}{l}\mathrm{R} \\
\mathrm{F} \\
\mathrm{R} \\
\mathrm{R}\end{array}$ & $\begin{array}{l}\mathbf{R} \\
\mathbf{R}\end{array}$ \\
\hline $\begin{array}{l}\text { Prunopyle hayesi } \\
\text { Carpocanarium papillosum } \\
\text { Calocyclas cf. semipolita } \\
\text { Cycladophora sp. } \\
\text { Cenosphaera } \text { sp. A } \\
\end{array}$ & & & & $\begin{array}{c}R \\
R-F \\
T \\
R-F \\
R\end{array}$ & $\begin{array}{c}R \\
R \\
R-F \\
R\end{array}$ & $\begin{array}{c}\mathrm{R} \\
\mathrm{R}-\mathrm{F} \\
\mathrm{R}-\mathrm{F}\end{array}$ & $\begin{array}{c}R \\
R-F \\
T \\
R\end{array}$ & $\begin{array}{c}\mathrm{R} \\
\mathrm{R}-\mathrm{F} \\
\mathrm{T} \\
\mathrm{T} \\
\mathrm{R}\end{array}$ & $\begin{array}{c}R \\
R \cdot F \\
R \\
R \\
R\end{array}$ & $\begin{array}{l}\mathrm{R} \\
\mathrm{R}\end{array}$ & $\begin{array}{l}\mathrm{R} \\
\mathrm{R}\end{array}$ & $\begin{array}{l}\mathrm{R} \\
\mathrm{R}\end{array}$ & $\begin{array}{c}\mathrm{F}-\mathrm{C} \\
\mathrm{F}\end{array}$ & $\begin{array}{l}\mathrm{R} \\
\mathrm{R}\end{array}$ & $\begin{array}{l}\mathrm{R} \\
\mathrm{R}\end{array}$ & $\begin{array}{c}\text { R-F } \\
\text { R }\end{array}$ & $\begin{array}{l}\mathrm{R} \\
\mathrm{T}\end{array}$ & $\begin{array}{l}\mathrm{F} \\
\mathrm{T}\end{array}$ \\
\hline $\begin{array}{l}\text { Spongodiscus craticulatus } \\
\text { Spongotrochus glacialis } \\
\text { Actinomma sp. B } \\
\text { Stauroxiphos communis } \\
\text { Dictyophimus gracilipes }\end{array}$ & & & & $\begin{array}{l}\mathrm{T} \\
\mathrm{R}\end{array}$ & $\begin{array}{c}\mathrm{R} \\
\mathrm{F} \\
\mathrm{F} \\
\mathrm{R}-\mathrm{F}\end{array}$ & $\begin{array}{l}\mathrm{R} \\
\mathrm{R}\end{array}$ & $\begin{array}{c}\mathrm{T} \\
\mathrm{R} \\
\mathrm{T} \cdot \mathrm{R} \\
\mathbf{R}\end{array}$ & $\begin{array}{l}\mathrm{T} \\
\mathrm{R} \\
\mathrm{T} \\
\mathrm{T} \\
\mathrm{R}\end{array}$ & $\begin{array}{l}\mathrm{T} \\
\mathrm{R} \\
\mathrm{R} \\
\mathrm{T} \\
\mathrm{R}\end{array}$ & $\begin{array}{l}\mathrm{T} \\
\mathrm{R} \\
\mathrm{T} \\
\mathrm{R} \\
\mathrm{R}\end{array}$ & $\mathrm{T}$ & $\mathbf{R}$ & $\mathrm{F}$ & $\mathrm{R}$ & $\mathrm{R}-\mathrm{F}$ & $\mathbf{R}$ & R-F & $\begin{array}{l}\mathrm{F} \\
\mathrm{R}-\mathrm{F}\end{array}$ \\
\hline $\begin{array}{l}\text { Velicucullus cf. oddgurneri } \\
\text { Gondwanaria sp. A } \\
\text { Cycladophora golli golli } \\
\text { Cycladophora golli regipileus } \\
\text { Gondwanaria deflandrei }\end{array}$ & & & & & $\begin{array}{c}\mathrm{T} \\
\mathrm{T} \cdot \mathrm{R} \\
\mathrm{R} \\
\mathrm{T}\end{array}$ & $\begin{array}{c}\mathrm{T} \\
\mathrm{R}-\mathrm{F} \\
\mathrm{R} \\
\mathrm{R}\end{array}$ & $\begin{array}{c}\mathrm{R} \\
\mathrm{T} \\
\mathrm{R}-\mathrm{F} \\
\mathrm{R}-\mathrm{F} \\
\mathrm{R}\end{array}$ & $\begin{array}{l}\mathrm{T} \\
\mathrm{R} \\
\mathrm{R} \\
\mathrm{R}\end{array}$ & $\begin{array}{l}\mathrm{R} \\
\mathrm{R} \\
\mathrm{R} \\
\mathrm{R} \\
\mathrm{R}\end{array}$ & $\begin{array}{l}\mathbf{R} \\
\mathbf{R} \\
\end{array}$ & $\begin{array}{c}\mathrm{R} \\
\mathrm{F}-\mathrm{C} \\
\mathrm{R}\end{array}$ & $\mathrm{F}$ & $\begin{array}{l}\mathrm{R} \\
\mathrm{F} \\
\mathrm{C}\end{array}$ & $\begin{array}{l}\mathrm{C} \\
\mathrm{R}\end{array}$ & $\begin{array}{l}\mathrm{T} \\
\mathrm{T} \\
\mathrm{C}\end{array}$ & $\begin{array}{l}\mathrm{T} \\
\mathrm{F}\end{array}$ & $\begin{array}{l}\mathrm{R} \\
\mathrm{T} \\
\mathrm{F}\end{array}$ & $\begin{array}{l}\text { R } \\
\text { F }\end{array}$ \\
\hline $\begin{array}{l}\text { Dendrospyris rhodospyroides } \\
\text { Cyrtocapsella japonica } \\
\text { Saccospyris antarctica } \\
\text { Prunopyle tetrapila } \\
\text { Heliodiscus sp. A } \\
\end{array}$ & & & & & & $\begin{array}{c}R \\
R-F\end{array}$ & $\begin{array}{l}\mathrm{R} \\
\mathrm{T} \\
\mathbf{R}\end{array}$ & $\begin{array}{c}\text { T-R } \\
\mathbf{R} \\
\mathrm{T} \\
\mathbf{R}\end{array}$ & $\begin{array}{l}\mathrm{R} \\
\mathrm{T} \\
\mathrm{T} \\
\mathrm{T}\end{array}$ & $\begin{array}{c}R \\
R \\
T-R \\
R \\
\end{array}$ & $\begin{array}{l}\mathrm{R} \\
\mathrm{R}\end{array}$ & $\begin{array}{l}\mathrm{R} \\
\mathrm{T}\end{array}$ & $\begin{array}{l}\mathrm{R} \\
\mathrm{R}\end{array}$ & $\mathbf{R}$ & $\begin{array}{l}\mathrm{R} \\
\mathbf{R} \\
\mathrm{T}\end{array}$ & $\begin{array}{l}\mathrm{R} \\
\mathrm{R} \\
\mathrm{T}\end{array}$ & $\begin{array}{l}\mathrm{R} \\
\mathrm{R}\end{array}$ & $\mathrm{R}$ \\
\hline $\begin{array}{l}\text { Actinomma sp. A } \\
\text { Disolenia spp. } \\
\text { Corythomelissa horrida } \\
\text { Stichophormis sp. } \\
\text { Eucyrtidium calvertense gr. }\end{array}$ & & & & & & & & $\mathrm{R}$ & $\begin{array}{c}\mathrm{R} \\
\mathrm{R}-\mathrm{F}\end{array}$ & $\begin{array}{r}\mathbf{R} \\
\mathbf{R}\end{array}$ & $\begin{array}{l}\mathrm{R} \\
\mathrm{T} \\
\mathrm{T}\end{array}$ & $\mathrm{T}$ & $\begin{array}{l}\mathrm{T} \\
\mathrm{T} \\
\mathrm{F} \\
\mathrm{R} \\
\mathrm{F}\end{array}$ & $\begin{array}{l}\mathrm{T} \\
\mathrm{T}\end{array}$ & $\begin{array}{l}\mathrm{T} \\
\mathrm{T}\end{array}$ & T-R & $\mathrm{T}$ & $\mathrm{R}$ \\
\hline $\begin{array}{l}\text { Eucyrtidium punctatum } \\
\text { Hexacontium spp. } \\
\text { Thyrsocyrtis clausa } \\
\text { Lamprocyrtis (?) cf. hannai } \\
\text { Pterocanium } \text { sp. }\end{array}$ & & & & & & & & & & & & & & & & & & \\
\hline $\begin{array}{l}\text { Anthocyrtidium sp. } \\
\text { Trissocylid sp. A } \\
\text { Eucyrtidium sp. A } \\
\text { Lychnocanoma sp. B } \\
\text { Cycladophora humerus }\end{array}$ & & & & & & & & t & & & & & & & & & & \\
\hline $\begin{array}{l}\text { Dendrospyris megalocephalis } \\
\text { Actinomma golownini } \\
\text { Antarctissa deflandrei } \\
\text { Stylosphaera sp. A } \\
\text { Prunopyle titan }\end{array}$ & & & & & & & & & & & & & & & & & & \\
\hline $\begin{array}{l}\text { Spongomelissa dilli } \\
\text { Lithocarpium polycantha } \\
\text { Cornutella clathrata } \\
\text { Didymocyrtis sp. } \\
\text { Cycladophora spongothorax }\end{array}$ & & & & & & & & & & & & & 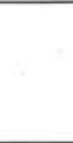 & & & & & \\
\hline lerid sp. B & & & & & & & & & & & & & & & & & & \\
\hline
\end{tabular}

Note. Abundance: $\mathrm{A}=$ abundant, $\mathrm{C}=$ common, $\mathrm{F}=$ few, $\mathrm{R}=$ rare, and $\mathrm{T}=$ trace. Preservation: $\mathrm{G}=$ good, $\mathrm{M}=$ moderate, and $\mathrm{P}=$ poor. 
Table 4 (continued).

E. punctatum

\begin{tabular}{|c|c|c|c|c|c|c|c|c|c|c|c|c|c|c|c|c|c|c|c|c|c|}
\hline $\begin{array}{l}15 \mathrm{H}- \\
\mathrm{CC}\end{array}$ & $\begin{array}{r}15 \mathrm{H}-6 \\
98-100\end{array}$ & $\begin{array}{r}15 \mathrm{H}-5 \\
98-100\end{array}$ & $\begin{array}{c}15 \mathrm{H}-4 \\
98-100\end{array}$ & $\begin{array}{c}15 \mathrm{H}-3 \\
98-100\end{array}$ & $\begin{array}{c}15 \mathrm{H}-2 \\
98-100\end{array}$ & $\begin{array}{c}15 \mathrm{H}-1 \\
98-100\end{array}$ & $\begin{array}{l}14 \mathrm{H}- \\
\mathrm{CC}\end{array}$ & $\begin{array}{r}14 \mathrm{H}-6 \\
98-100\end{array}$ & $\begin{array}{r}14 \mathrm{H}-5 \\
98-100\end{array}$ & $\begin{array}{r}14 \mathrm{H}-4 \\
98-100\end{array}$ & $\begin{array}{c}14 \mathrm{H} \cdot 3 \\
98-100\end{array}$ & $\begin{array}{c}14 \mathrm{H}-2 \\
98-100\end{array}$ & $\begin{array}{c}14 \mathrm{H}-1 \\
98-100\end{array}$ & $\begin{array}{l}13 \mathrm{H}- \\
\mathrm{CC}\end{array}$ & $\begin{array}{r}13 \mathrm{H}-7 \\
98-100\end{array}$ & $\begin{array}{r}13 \mathrm{H}-6 \\
98-100\end{array}$ & $\begin{array}{r}13 \mathrm{H}-5 \\
98-100\end{array}$ & $\begin{array}{l}13 \mathrm{H}-5 \\
32-36\end{array}$ & $\begin{array}{r}13 \mathrm{H}-4 \\
98-100\end{array}$ & $\begin{array}{l}13 \mathrm{H}-4 \\
32-34\end{array}$ & $\begin{array}{r}13 \mathrm{H}-3 \\
98-100\end{array}$ \\
\hline $\begin{array}{l}\text { A } \\
\text { G }\end{array}$ & $\begin{array}{c}\text { F-C } \\
\text { G }\end{array}$ & $\begin{array}{l}\text { F-C } \\
\text { M-G }\end{array}$ & $\begin{array}{l}\text { F-C } \\
\text { M-G }\end{array}$ & $\begin{array}{c}\text { F-C } \\
\text { M }\end{array}$ & $\begin{array}{c}\text { F-C } \\
\text { M }\end{array}$ & $\begin{array}{c}\text { F-C } \\
\text { M }\end{array}$ & $\begin{array}{l}\text { C } \\
\text { G }\end{array}$ & $\begin{array}{c}\text { F-C } \\
\text { M }\end{array}$ & $\begin{array}{c}C \\
M-G\end{array}$ & $\begin{array}{l}\text { F-C } \\
\text { M-G }\end{array}$ & $\underset{M-G}{F}$ & $\begin{array}{c}\text { R-F } \\
\text { M }\end{array}$ & B & $\begin{array}{c}\text { R-F } \\
\text { G }\end{array}$ & $\begin{array}{c}\mathrm{R} \\
\mathrm{M}-\mathrm{G}\end{array}$ & $\begin{array}{l}\mathrm{R} \\
\mathrm{M}\end{array}$ & $\begin{array}{c}R \\
M-P\end{array}$ & $\begin{array}{l}\mathrm{R} \\
\mathrm{M}\end{array}$ & B & $\begin{array}{c}\text { R-F } \\
\text { M }\end{array}$ & $\begin{array}{l}\mathrm{R} \\
\mathrm{M}\end{array}$ \\
\hline $\begin{array}{l}\mathrm{F} \\
\mathrm{R} \\
\mathrm{R}\end{array}$ & $\begin{array}{c}\text { F-C } \\
R \\
R \\
R\end{array}$ & $\begin{array}{l}\mathbf{R} \\
\mathrm{R} \\
\mathrm{R} \\
\mathrm{R}\end{array}$ & $\begin{array}{c}\mathrm{R} \\
\mathrm{T}-\mathrm{R} \\
\mathrm{R} \\
\mathrm{T}\end{array}$ & $\begin{array}{l}\mathrm{R} \\
\mathrm{R} \\
\mathrm{R} \\
\mathrm{R}\end{array}$ & $\begin{array}{l}\mathrm{R} \\
\mathrm{R}\end{array}$ & $\begin{array}{l}\mathrm{T} \\
\mathrm{R} \\
\mathrm{T} \\
\mathrm{T}\end{array}$ & $\begin{array}{c}\mathrm{F}-\mathrm{C} \\
\mathrm{R}-\mathrm{F} \\
\mathrm{R} \\
\mathrm{R}\end{array}$ & $\begin{array}{l}\dot{R} \\
\mathrm{~T} \\
\mathrm{~T}\end{array}$ & $\begin{array}{l}\mathrm{R} \\
\mathrm{R} \\
\mathrm{T} \\
\mathrm{T}\end{array}$ & $\begin{array}{l}\mathrm{T} \\
\mathrm{T} \\
\mathrm{R}\end{array}$ & $\begin{array}{l}\mathbf{R} \\
\mathrm{T}\end{array}$ & R & & $\begin{array}{c}\mathbf{R} \\
\mathbf{R}-\mathrm{T} \\
\mathrm{R}\end{array}$ & $\begin{array}{l}\mathrm{R} \\
\mathrm{T} \\
\mathrm{T}\end{array}$ & $\begin{array}{l}\mathrm{R} \\
\mathrm{T} \\
\mathrm{T}\end{array}$ & $\begin{array}{l}\mathbf{R} \\
\mathrm{T}\end{array}$ & $\mathrm{T}$ & $\mathrm{T}$ & $\mathrm{T}$ & $\begin{array}{l}\mathrm{T} \\
\mathrm{T}\end{array}$ \\
\hline F & R & R & R & $\bar{R}$ & R & R & $\mathrm{F}$ & T-R & & $T$ & R & R & & $R$ & $\mathrm{R}$ & T-R & & $\mathbf{R}$ & & $T$ & $T$ \\
\hline $\begin{array}{l}R \\
R \\
R\end{array}$ & $\begin{array}{l}\mathrm{R} \\
\mathrm{R}\end{array}$ & $\begin{array}{l}\mathbf{R} \\
\mathbf{R}\end{array}$ & $\begin{array}{l}\mathrm{R} \\
\mathrm{R}\end{array}$ & $\begin{array}{l}\mathbf{R} \\
\mathrm{T}\end{array}$ & $\begin{array}{l}\mathrm{R} \\
\mathrm{R} \\
\mathrm{R}\end{array}$ & $\begin{array}{l}\mathrm{R} \\
\mathrm{R} \\
\mathrm{T}\end{array}$ & $\begin{array}{l}\mathrm{R} \\
\mathrm{R} \\
\mathrm{R}\end{array}$ & $\begin{array}{l}\mathrm{T} \\
\mathrm{R}\end{array}$ & $\begin{array}{l}T \\
R\end{array}$ & $\mathrm{~T}$ & & $T$ & & $\begin{array}{l}\mathrm{R} \\
\mathrm{R} \\
\mathrm{R}\end{array}$ & $T$ & $\mathrm{~T}$ & $T$ & $\mathrm{~T}$ & & $\mathbf{R}$ & $\mathrm{T}$ \\
\hline $\mathbf{R}$ & $\mathrm{T}$ & R & R & R & $T$ & & R & & R & & & & & & & & & & & & \\
\hline $\mathbf{R}$ & T-R & $\mathbf{R}$ & R & $\begin{array}{l}\mathrm{T} \\
\mathrm{R}\end{array}$ & R & R & $\mathbf{R}$ & $\mathrm{T}$ & $\mathrm{T}$ & & $\mathrm{T}$ & R & & R & $\begin{array}{l}\mathrm{T} \\
\mathrm{R}\end{array}$ & $\mathrm{T}$ & $T$ & $\mathrm{~T}$ & & R & $\begin{array}{l}\mathrm{T} \\
\mathrm{R}\end{array}$ \\
\hline R & $\begin{array}{l}\mathrm{R} \\
\mathrm{R}\end{array}$ & $\begin{array}{l}T \\
T\end{array}$ & $\begin{array}{l}\mathrm{T} \\
\mathrm{T} \\
\mathrm{T} \\
\mathrm{R}\end{array}$ & $\begin{array}{l}\mathrm{T} \\
\mathrm{T} \\
\mathrm{T}\end{array}$ & $\mathrm{T}$ & $\begin{array}{l}T \\
T \\
T \\
T\end{array}$ & $\begin{array}{l}\mathrm{R} \\
\mathrm{T} \\
\mathrm{R}\end{array}$ & & $\begin{array}{l}\mathrm{T} \\
\mathrm{T}\end{array}$ & & $\begin{array}{l}\mathrm{T} \\
\mathrm{T}\end{array}$ & $\begin{array}{l}\mathrm{R} \\
\mathrm{T} \\
\mathrm{T}\end{array}$ & & R & $\begin{array}{l}\mathrm{T} \\
\mathrm{R} \\
\mathrm{T}\end{array}$ & $\begin{array}{l}\mathrm{R} \\
\mathrm{R} \\
\mathrm{T}\end{array}$ & $\mathrm{T}$ & $\begin{array}{l}\mathrm{T} \\
\mathrm{R} \\
\mathrm{T}\end{array}$ & & R & $\begin{array}{l}\mathrm{T} \\
\mathrm{T}\end{array}$ \\
\hline $\begin{array}{l}\mathrm{F} \\
\mathrm{R} \\
\mathrm{T}\end{array}$ & $\mathrm{R}$ & $\begin{array}{l}\mathrm{R} \\
\mathrm{R} \\
\mathrm{R} \\
\mathrm{R} \\
\mathrm{R}\end{array}$ & $\begin{array}{c}\text { R-F } \\
\text { R-F } \\
\text { R } \\
\text { R } \\
\text { R }\end{array}$ & $\begin{array}{l}\mathrm{R} \\
\mathrm{R} \\
\mathrm{R} \\
\mathrm{R} \\
\mathrm{R}\end{array}$ & $\begin{array}{c}\text { R-F } \\
\text { R-F } \\
\text { R } \\
\text { R } \\
\text { R }\end{array}$ & $\begin{array}{l}\mathrm{R} \\
\mathrm{F} \\
\mathrm{R} \\
\mathrm{R} \\
\mathrm{R}\end{array}$ & $\begin{array}{c}\text { R-F } \\
\text { R } \\
\text { R }\end{array}$ & $\begin{array}{l}\mathrm{R} \\
\mathrm{R} \\
\mathrm{R} \\
\mathrm{T} \\
\mathrm{R}\end{array}$ & $\begin{array}{l}\mathrm{R} \\
\mathrm{R} \\
\mathrm{R} \\
\mathrm{T} \\
\mathrm{R}\end{array}$ & $\begin{array}{l}\mathrm{R} \\
\mathrm{R} \\
\mathrm{R} \\
\mathrm{T} \\
\mathrm{R}\end{array}$ & $\begin{array}{l}\mathrm{R} \\
\mathrm{T} \\
\mathrm{R} \\
\mathrm{R} \\
\mathrm{R}\end{array}$ & $\begin{array}{l}\mathrm{R} \\
\mathrm{R} \\
\mathrm{R} \\
\mathrm{R} \\
\mathrm{T}\end{array}$ & & $\begin{array}{c}\text { R-F } \\
\text { R } \\
\text { R }\end{array}$ & $\begin{array}{c}\mathrm{R} \\
\mathrm{R} \\
\mathrm{R} \\
\mathrm{R} \\
\mathrm{T}-\mathrm{R}\end{array}$ & $\begin{array}{c}\text { R-F } \\
\mathrm{R} \\
\mathrm{R} \\
\mathrm{R} \\
\mathrm{T}\end{array}$ & $\begin{array}{l}\mathbf{R} \\
\mathbf{R} \\
\mathrm{T} \\
\mathbf{T}\end{array}$ & $\begin{array}{l}\mathbf{R} \\
\mathbf{R} \\
\mathbf{R} \\
\mathbf{R} \\
\mathbf{T}\end{array}$ & & $\begin{array}{c}\mathrm{R} \\
\mathrm{R} \\
\mathrm{R}-\mathrm{F} \\
\mathrm{R} \\
\mathrm{R}\end{array}$ & $\begin{array}{l}\mathbf{R} \\
\mathbf{R} \\
\mathbf{R} \\
\mathrm{T} \\
\mathrm{T}\end{array}$ \\
\hline $\begin{array}{l}\mathrm{R} \\
\mathrm{R}\end{array}$ & $\begin{array}{l}\mathbf{R} \\
\mathbf{R}\end{array}$ & $\begin{array}{l}\mathrm{R} \\
\mathrm{R}\end{array}$ & $\begin{array}{l}\mathbf{R} \\
\mathrm{R}\end{array}$ & $\begin{array}{l}\mathbf{R} \\
\mathbf{R} \\
\mathrm{T}\end{array}$ & $\begin{array}{l}\mathbf{R} \\
\mathbf{R} \\
\mathrm{T}\end{array}$ & $\begin{array}{l}\mathrm{R} \\
\mathrm{R}\end{array}$ & $\begin{array}{l}\text { R } \\
\text { R }\end{array}$ & $\begin{array}{l}\mathrm{R} \\
\mathrm{T} \\
\mathrm{T}\end{array}$ & R & R & $R$ & $\begin{array}{l}R \\
R\end{array}$ & & R-F & $\begin{array}{l}\text { R } \\
T \\
T\end{array}$ & $\begin{array}{l}\mathrm{T} \\
\mathrm{R}\end{array}$ & R & $\begin{array}{l}\mathbf{R} \\
\mathbf{R}\end{array}$ & & $T$ & $\begin{array}{c}T \\
T-R\end{array}$ \\
\hline
\end{tabular}

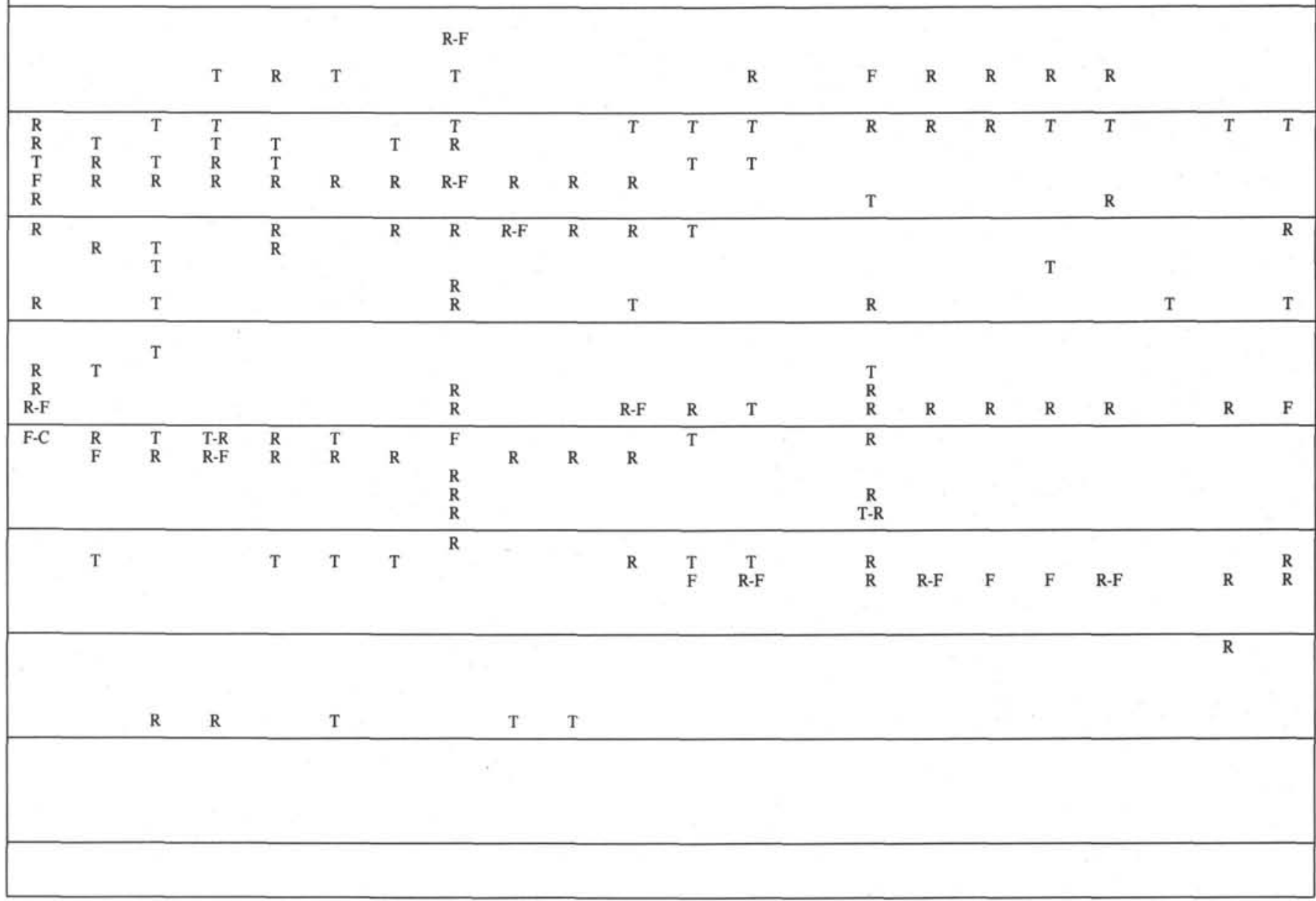




\section{A. ABELMANN}

Table 4. (continued).

\begin{tabular}{|c|c|c|c|c|c|c|c|c|c|c|c|c|c|c|c|c|c|c|}
\hline \multirow{2}{*}{$\begin{array}{l}\text { Zones } \\
\text { Core, section } \\
\text { Interval }(\mathrm{cm})\end{array}$} & \multicolumn{4}{|c|}{ C. humerus } & \multicolumn{6}{|c|}{ upper $A$. golownini } & \multicolumn{8}{|c|}{ C. spongothorax } \\
\hline & $\begin{array}{l}13 \mathrm{H}-3 \\
32-36\end{array}$ & $\begin{array}{c}13 \mathrm{H}-2 \\
98-100\end{array}$ & $\begin{array}{c}13 \mathrm{H}-1 \\
98-100\end{array}$ & $\begin{array}{l}13 \mathrm{H}-1 \\
32-36\end{array}$ & $\begin{array}{c}12 \mathrm{H}- \\
\mathrm{CC}\end{array}$ & $\begin{array}{r}12 \mathrm{H}-5 \\
98-100 \\
\end{array}$ & $\begin{array}{c}12 \mathrm{H}-4 \\
98-100\end{array}$ & $\begin{array}{c}12 \mathrm{H}-3 \\
98-100\end{array}$ & $\begin{array}{c}12 \mathrm{H}-2 \\
98-100\end{array}$ & $\begin{array}{c}12 \mathrm{H}-1 \\
98-100 \\
\end{array}$ & $\begin{array}{c}11 \mathrm{H}- \\
\mathrm{CC}\end{array}$ & $\begin{array}{r}11 \mathrm{H}-6 \\
98-100\end{array}$ & $\begin{array}{c}11 \mathrm{H}-5 \\
98-100\end{array}$ & $\begin{array}{c}11 \mathrm{H}-4 \\
98-100\end{array}$ & $\begin{array}{c}11 \mathrm{H}-3 \\
98-100\end{array}$ & $\begin{array}{c}11 \mathrm{H}-2 \\
98-100\end{array}$ & $\begin{array}{c}11 \mathrm{H}-1 \\
98-100\end{array}$ & $\begin{array}{c}10 \mathrm{H}- \\
\mathrm{CC}\end{array}$ \\
\hline $\begin{array}{l}\text { Abundance } \\
\text { Preservation }\end{array}$ & $\begin{array}{c}\mathrm{R} \\
\mathrm{M}-\mathrm{P}\end{array}$ & $\begin{array}{l}\mathrm{F} \\
\mathrm{M}\end{array}$ & $\begin{array}{l}\mathrm{F} \\
\mathrm{M}\end{array}$ & $\begin{array}{l}\mathrm{R} \\
\mathrm{P}\end{array}$ & $\begin{array}{l}\mathrm{C} \\
\mathrm{G}\end{array}$ & $\begin{array}{c}\text { F-C } \\
\text { M }\end{array}$ & $\begin{array}{c}\mathrm{F}-\mathrm{C} \\
\mathrm{M}\end{array}$ & $\begin{array}{c}\text { F-C } \\
M\end{array}$ & $\begin{array}{c}\mathrm{C} \\
\mathrm{M}-\mathrm{G}\end{array}$ & $\begin{array}{l}\text { F-C } \\
\text { M-G }\end{array}$ & $\begin{array}{l}F \\
G\end{array}$ & $\begin{array}{l}\text { F-C } \\
\text { M-G }\end{array}$ & $\begin{array}{c}\text { F-C } \\
G\end{array}$ & $\begin{array}{c}\text { F-C } \\
M\end{array}$ & $\underset{M-G}{F}$ & $\stackrel{\mathrm{R}}{\mathrm{M}-\mathrm{G}}$ & $\stackrel{\text { R }}{M-G}$ & $\begin{array}{l}F \\
G\end{array}$ \\
\hline $\begin{array}{l}\text { Cyrtocapsella tetrapera } \\
\text { Cyrtocapsella longithorax } \\
\text { Lithomelissa cf. ehrenbergi } \\
\text { Amphistylus angelinus } \\
\text { Cycladophora antiqua }\end{array}$ & $\mathrm{T}$ & & $\mathbf{R}$ & $\mathbf{R}$ & & $\mathrm{T}$ & $\mathbf{R}$ & & & & & & & $\begin{array}{l}\mathrm{R} \\
\mathrm{R}\end{array}$ & $\mathrm{T}$ & & & $\mathrm{T}$ \\
\hline $\begin{array}{l}\text { Prunopyle sp. A } \\
\text { Velicucullus altus } \\
\text { Circodiscus ellipticus } \\
\text { Stylatractus neptunus } \\
\text { Prunopyle sp. D } \\
\end{array}$ & $\mathrm{T}$ & $\mathrm{R}$ & $\mathbf{R}$ & $\mathbf{R}$ & $\begin{array}{l}\mathrm{R} \\
\mathrm{R}\end{array}$ & $\mathrm{T}$ & & $\mathrm{T}$ & $\mathbf{R}$ & $\mathrm{T}$ & $\mathbf{R}$ & $\mathrm{T}$ & $\mathrm{T}$ & & & & & \\
\hline $\begin{array}{l}\text { Hexacontium } \text { cf. enthacanthum } \\
\text { Corythospyris fiscella } \\
\text { Tripilidium clavipes } \\
\text { Gondwanaria japonica } \\
\text { Cycladophora conica }\end{array}$ & T & $\mathrm{T}$ & & $\mathrm{T}$ & $\mathbf{R}$ & & & $\mathbf{R}$ & $\mathbf{R}$ & $\mathbf{R}$ & & $\mathbf{R}$ & $\begin{array}{l}\mathrm{T} \\
\mathrm{R}\end{array}$ & $\mathbf{R}$ & $\mathbf{R}$ & $\mathrm{R}$ & $\mathbf{R}$ & $\mathbf{R}$ \\
\hline $\begin{array}{l}\text { Peripyramis circumtexta } \\
\text { Druppatractus hastatus } \\
\text { Ceratocyrtis mashae } \\
\text { Dendrospyris stabilis } \\
\text { Acrosphaera } \text { spp. }\end{array}$ & $T$ & $\begin{array}{l}\mathrm{T} \\
\mathrm{R} \\
\mathrm{T}\end{array}$ & $\mathrm{T}$ & $\mathrm{T}$ & $\begin{array}{l}\mathrm{R} \\
\mathrm{T}\end{array}$ & & $\mathbf{R}$ & $\begin{array}{l}\mathrm{R} \\
\mathrm{T}\end{array}$ & & & $\mathbf{R}$ & & & & & & & $\mathrm{T}$ \\
\hline $\begin{array}{l}\text { Siphocampe arachnea gr. } \\
\text { Antarctissa robusta } \\
\text { Eucyrtidium cienkowskii gr. } \\
\text { Ceratocyrtis stigi } \\
\text { Prunopyle sp. B }\end{array}$ & $\begin{array}{l}\mathrm{R} \\
\mathrm{R} \\
\mathrm{T}\end{array}$ & $\begin{array}{l}\mathrm{R} \\
\mathrm{R} \\
\mathrm{R} \\
\mathrm{R} \\
\mathrm{R}\end{array}$ & $\begin{array}{l}\mathrm{R} \\
\mathrm{R} \\
\mathrm{F} \\
\mathrm{R} \\
\mathrm{R}\end{array}$ & $\begin{array}{l}\mathrm{R} \\
\mathrm{R} \\
\mathrm{R}\end{array}$ & $\begin{array}{l}\mathrm{R} \\
\mathrm{R} \\
\mathrm{R}\end{array}$ & $\begin{array}{l}\mathrm{R} \\
\mathrm{R} \\
\mathrm{R} \\
\mathrm{R} \\
\mathrm{R}\end{array}$ & $\begin{array}{l}\mathrm{R} \\
\mathrm{T} \\
\mathrm{R} \\
\mathrm{R} \\
\mathrm{R}\end{array}$ & $\begin{array}{l}\mathrm{R} \\
\mathrm{R} \\
\mathrm{R} \\
\mathrm{R} \\
\mathrm{R}\end{array}$ & $\begin{array}{l}\mathbf{R} \\
\mathbf{R}\end{array}$ & $\begin{array}{l}\mathrm{R} \\
\mathrm{R} \\
\mathrm{R} \\
\mathrm{R}\end{array}$ & $\begin{array}{l}\mathbf{R} \\
\mathbf{R}\end{array}$ & $\begin{array}{l}\mathbf{R} \\
\mathbf{R}\end{array}$ & $\begin{array}{l}\mathrm{T} \\
\mathrm{R} \\
\mathrm{R} \\
\mathrm{R}\end{array}$ & $\begin{array}{l}\mathrm{R} \\
\mathrm{R} \\
\mathrm{R}\end{array}$ & $\mathrm{T}$ & & $\mathrm{T}$ & \\
\hline $\begin{array}{l}\text { Prunopyle hayesi } \\
\text { Carpocanarium papillosum } \\
\text { Calocyclas cf. semipolita } \\
\text { Cycladophora sp. } \\
\text { Cenosphaera } \text { sp. A } \\
\end{array}$ & $\begin{array}{l}\mathrm{T} \\
\mathrm{R}\end{array}$ & $\begin{array}{l}\mathrm{R} \\
\mathrm{R}\end{array}$ & $\begin{array}{l}\mathrm{R} \\
\mathrm{R}\end{array}$ & $\mathbf{R}$ & $\begin{array}{l}\mathrm{R} \\
\mathrm{T}\end{array}$ & $\mathrm{R}$ & $\mathrm{R}$ & $\mathrm{R}$ & $\mathrm{R}$ & $\mathbf{R}$ & $\mathbf{R}$ & & & & & & & $\mathbf{R}$ \\
\hline $\begin{array}{l}\text { Spongodiscus craticulatus } \\
\text { Spongotrochus glacialis } \\
\text { Actinomma sp. B } \\
\text { Stauroxiphos communis } \\
\text { Dictyophimus gracilipes }\end{array}$ & & $\mathrm{T}$ & R-F & $\mathrm{T}$ & R-F & & $\mathrm{T}$ & & & & & $\mathrm{T}$ & $\mathbf{R}$ & $\mathrm{T}$ & & & & $\mathrm{T}$ \\
\hline $\begin{array}{l}\text { Velicucullus cf. oddgurneri } \\
\text { Gondwanaria sp. A } \\
\text { Cycladophora golli golli } \\
\text { Cycladophora golli regipileus } \\
\text { Gondwanaria deflandrei } \\
\end{array}$ & $\mathrm{T}$ & $\mathrm{T}$ & $\mathrm{T}$ & & $\mathbf{R}$ & R-F & $\mathbf{R}$ & & $\mathbf{R}$ & $\mathrm{T}$ & $T$ & $\begin{array}{l}\mathrm{T} \\
\mathrm{R}\end{array}$ & $\begin{array}{l}\mathrm{T} \\
\mathrm{R} \\
\end{array}$ & $\mathbf{T}$ & $T$ & $\mathrm{~T}$ & & $\mathrm{~T}$ \\
\hline $\begin{array}{l}\text { Dendrospyris rhodospyroides } \\
\text { Cyrtocapsella japonica } \\
\text { Saccospyris antarctica } \\
\text { Prunopyle tetrapila } \\
\text { Heliodiscus sp. A } \\
\end{array}$ & $\mathrm{T}$ & $\mathrm{T}$ & $\begin{array}{l}\mathrm{R} \\
\mathrm{T}\end{array}$ & $\mathbf{R}$ & $\begin{array}{l}\mathbf{R} \\
\mathbf{R}\end{array}$ & & & $\mathrm{T}$ & $T$ & $\begin{array}{l}\mathrm{R} \\
\mathrm{T}\end{array}$ & $\mathrm{R}$ & R-F & R-F & R-F & $\mathbf{R}$ & $\begin{array}{l}\mathbf{R} \\
\mathrm{T}\end{array}$ & $\begin{array}{l}\mathrm{T} \\
\mathrm{T}\end{array}$ & $\begin{array}{l}\mathrm{R} \\
\mathrm{R}\end{array}$ \\
\hline $\begin{array}{l}\text { Actinomma sp. A } \\
\text { Disolenia spp. } \\
\text { Corythomelissa horrida } \\
\text { Stichophormis sp. } \\
\text { Eucyrtidium calvertense gr. }\end{array}$ & $\mathbf{R}$ & & & $\mathrm{T}$ & $\begin{array}{l}\mathrm{T} \\
\mathrm{R} \\
\end{array}$ & $\mathbf{R}$ & $\mathrm{F}$ & & & & $\mathbf{R}$ & $\mathbf{R}$ & $\mathbf{R}$ & $\mathbf{R}$ & $\mathrm{T}$ & $\mathrm{T}$ & $\mathrm{T}$ & $\mathbf{R}$ \\
\hline $\begin{array}{l}\text { Eucyrtidium punctatum } \\
\text { Hexacontium spp. } \\
\text { Thyrsocyrtis clausa } \\
\text { Lamprocyrtis (?) cf. hannai } \\
\text { Pterocanium sp. } \\
\end{array}$ & & & $\mathrm{T}$ & $\mathrm{T}$ & $\mathbf{R}$ & & & & & $\mathbf{R}$ & & $\mathbf{R}$ & $\mathbf{R}$ & $\mathbf{R}$ & & & $\mathrm{T}$ & \\
\hline $\begin{array}{l}\text { Anthocyrtidium sp. } \\
\text { Trissocylid sp. A } \\
\text { Eucyrtidium sp. A } \\
\text { Lychnocanoma sp. B } \\
\text { Cycladophora humerus }\end{array}$ & $\mathrm{T}$ & $\begin{array}{l}\mathrm{R} \\
\mathrm{R} \\
\mathrm{R} \\
\mathrm{R}\end{array}$ & $\begin{array}{l}\mathrm{R} \\
\mathrm{T} \\
\mathrm{R}\end{array}$ & $\begin{array}{l}\mathrm{R} \\
\mathrm{T} \\
\mathrm{R}\end{array}$ & $\begin{array}{l}\mathrm{F} \\
\mathrm{F}\end{array}$ & $\begin{array}{l}\mathrm{R} \\
\mathrm{R} \\
\mathrm{F} \\
\mathrm{F}\end{array}$ & $\begin{array}{l}\mathrm{R} \\
\mathrm{F}\end{array}$ & $\begin{array}{l}\mathrm{T} \\
\mathrm{R} \\
\mathrm{F}\end{array}$ & $\begin{array}{c}\mathrm{T} \\
\mathrm{R}-\mathrm{F} \\
\mathrm{R}-\mathrm{F}\end{array}$ & $\begin{array}{l}\mathrm{T} \\
\mathrm{R}-\mathrm{F}\end{array}$ & $\mathbf{R}$ & $\mathbf{R}$ & $\mathbf{R}$ & $\mathbf{R}$ & $\mathbf{R}$ & $\mathrm{T}$ & & $\mathrm{T}$ \\
\hline $\begin{array}{l}\text { Dendrospyris megalocephalis } \\
\text { Actinomma golownini } \\
\text { Antarctissa deflandrei } \\
\text { Stylosphaera sp. A } \\
\text { Prunopyle titan }\end{array}$ & & & $\mathrm{R}$ & $\begin{array}{l}T \\
T\end{array}$ & $\begin{array}{l}\mathrm{R} \\
\mathrm{R} \\
\mathrm{R} \\
\mathrm{R}\end{array}$ & $\begin{array}{c}\mathrm{R} \\
\mathrm{R} \\
\mathrm{F}-\mathrm{C} \\
\mathrm{R}\end{array}$ & $\begin{array}{c}\mathrm{T} \\
\mathrm{R} \\
\mathrm{F}-\mathrm{C} \\
\mathrm{T} \\
\mathrm{R}\end{array}$ & $\begin{array}{c}R \\
R \\
C-A\end{array}$ & $\begin{array}{l}\mathrm{R} \\
\mathrm{R} \\
\mathrm{C}\end{array}$ & $\begin{array}{l}\mathrm{R} \\
\mathrm{F} \\
\mathrm{T}\end{array}$ & $\underset{\mathrm{R}-\mathrm{F}}{\mathrm{R}}$ & $\begin{array}{l}\mathrm{F} \\
\mathrm{R}\end{array}$ & $\begin{array}{l}\mathrm{T} \\
\mathrm{F} \\
\mathrm{R}\end{array}$ & $\begin{array}{l}\mathrm{T} \\
\mathrm{F}\end{array}$ & $\mathrm{T}$ & $\mathrm{R}$ & $\mathrm{R}$ & $\begin{array}{l}\mathrm{R} \\
\mathrm{R}\end{array}$ \\
\hline $\begin{array}{l}\text { Spongomelissa dilli } \\
\text { Lithocarpium polycantha } \\
\text { Cornutella clathrata } \\
\text { Didymocyrtis sp. } \\
\text { Cycladophora spongothorax } \\
\end{array}$ & & & & & $\mathbf{R}$ & $\mathrm{R}$ & $\begin{array}{c}R \\
T-R \\
R\end{array}$ & $\begin{array}{l}\mathrm{R} \\
\mathrm{T}\end{array}$ & $\begin{array}{l}R \\
T\end{array}$ & $\begin{array}{l}T \\
T\end{array}$ & $\begin{array}{l}\mathrm{R} \\
\mathrm{R} \\
\end{array}$ & $\begin{array}{c}\mathrm{R} \\
\mathrm{R} \\
\mathrm{R}-\mathrm{F}\end{array}$ & $\begin{array}{c}R \\
T-R \\
R-F\end{array}$ & $\begin{array}{l}\mathbf{R} \\
\mathbf{R} \\
\mathbf{R} \\
\end{array}$ & $\begin{array}{l}\mathrm{R} \\
\mathrm{R} \\
\end{array}$ & $\begin{array}{l}\mathrm{T} \\
\mathbf{R} \\
\end{array}$ & $\begin{array}{l}\mathrm{T} \\
\mathrm{R} \\
\end{array}$ & R-F \\
\hline Collosphaerid sp. B & & & & & & & & & & & & & & & & $\mathrm{T}$ & & $\mathrm{T}$ \\
\hline
\end{tabular}




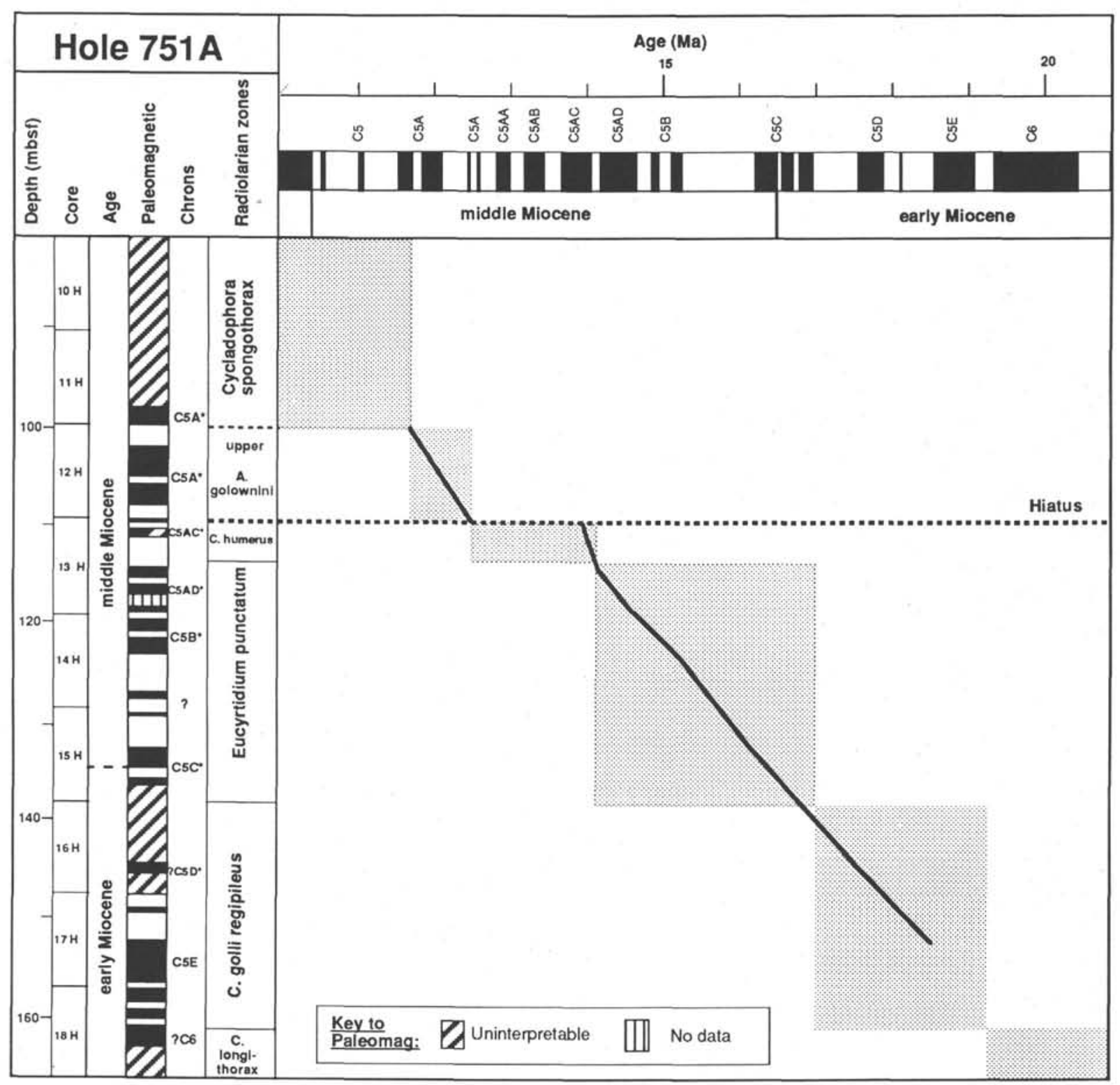

Figure 7. Age-depth interpretation of the early to middle Miocene time interval of Hole 751A based on the geomagnetic polarity pattern (thick line) of Heider (this volume) and the radiolarian zonation. Stippled boxes indicate depth/age range of radiolarian zones. Horizontal dashed lines indicate location of hiatus. Geomagnetic chron designations were taken from Schlich, Wise, et al. (1990, "Site 751" chapter), except those marked by asterisks. Asterisk-marked chron designations indicate revisions based on the radiolarian biostratigraphic age determination. Stratigraphic ranges of radiolarian zones are correlated to the standard geomagnetic time scale of Berggren et al. (1985).

The boundary between the upper A. golownini Subzone and middle to late Miocene Cycladophora spongothorax Zone, marked by the FAD of $C$. spongothorax, was placed between Sample 120-751A-12H-1, 134-138 cm, and Section $120-751 \mathrm{~A}-11 \mathrm{H}-\mathrm{CC}$ (99.7-101 mbsf). This boundary was placed at approximately $11.8 \mathrm{Ma}$ (Abelmann, 1990).

\section{DISCUSSION}

During Leg 120, lower to middle Miocene sediments were recovered at Sites 747,748 , and 751 . They can be dated using the occurrence of radiolarian stratigraphic marker species and the paleomagnetic polarity pattern measured by Heider (this volume) and Inokuchi (this volume). The zonation used relies on a radiolarian biostratigraphic zonation (correlated with the geomagnetic time scale) that was recently developed for Leg 113 Sites 689 and 690 recovered at Maud Rise (Atlantic sector of the Southern Ocean) (Abelmann, 1990). In particular, zones in the middle Miocene time interval, younger than $15 \mathrm{Ma}$, could be correlated accurately with the geomagnetic time scale at both Leg 113 Maud Rise sites. The radiolarian zones were also correlated with a diatom biostratigraphic zonation (Gersonde et al., 1990), and the proposed age interpretation was supported by the biostratigraphic study of Leg 119 materials accomplished by Baldauf and Barron (1991).

Because the geomagnetic polarity pattern for Leg 120 is problematic, as a result of the presence of disconformities and gaps in the geomagnetic record, part of the age interpretation 
of the investigated middle Miocene sediment sequences of the studied Leg 120 Holes 747 A, 748B, and 751A was based on the zonal age calibration established on the Leg 113 materials. As a consequence, part of the chronostratigraphic interpretation of the paleomagnetic polarity pattern given in Schlich, Wise, et al. (1989) was revised.

The age assignment of the radiolarian zonation for the lower Miocene proposed by Abelmann (1990) was improved and emended by this study because a more expanded lower Miocene sequences were recovered in the Leg 120 holes investigated. The lower Miocene gathered at Leg 113 Holes 689B and 690B (Figs. 2 and 8 ) is reduced by the occurrence of disconformities, and the interpretation of the geomagnetic polarity pattern measured by Spieß (1990) was problematical. In this study, most lower Miocene radiolarian zones could be correlated to an older time range, compared with the age calibration proposed by Abelmann (1990) (Figs. 2 and 8). As a consequence, the improved and emended age calibration proposed in this study could be used for a reinterpretation of the geomagnetic record and the stratigraphic age assignment of the early Miocene sequences of Leg 113 Holes 689B and 690B (Fig. 8). However, the occurrence of disconformities and intervals lacking geomagnetic data in the investigated Leg 120 lower Miocene sequences prevents an accurate determination of the zonal boundary ages. In particular, the age of the boundary between the $C$. antiqua and the $C$. longithorax zones could not be delineated very well (Figs. 2 and 8).

As an additional stratigraphic marker species in the lower part of the lower Miocene, the cosmopolitan species Cyrtocapsella tetrapera was used for stratigraphic correlation. Based on the occurrence of this species, which was not encountered in the Leg 113 materials investigated (Abelmann, 1990), the base of the $C$. antiqua Zone, which is placed by Abelmann (1990) in Chron C6 at approximately 19.9-19.4 Ma, can preliminarily be placed in an interval ranging from the top of Subchron C6B to the reversed interval between the normal events of Subchron C6AA at 22.6-22.2 Ma (see the discussion of the $C$. antiqua Zone, this chapter). A different age determination was determined for the FAD of the diatom species Thalassiosira fraga, which occurs nearly in the same core depth as the FADs of $C$. tetrapera and $C$. antiqua (Harwood and Maruyama, this volume). Harwood and Maruyama (this volume) placed the FAD of $T$. fraga in the time interval between approximately 20.8 and $21.2 \mathrm{Ma}$ older than Baldauf and Barron (1991), who placed the FAD of $T$. fraga between 20.2 and 21.0 Ma. Up to now, the age determination of the bases of the Cycladophora antiqua and the $T$. fraga zones are still under discussion.

In Hole $747 \mathrm{~A}$, not much conflict between the radiolarian biostratigraphic age interpretation and the age assignment based on the interpretation of the geomagnetic polarity record according to Schlich, Wise, et al. (1989) was found. Only one geomagnetic chron designation in the lower portion of the $C$. humerus Zone was revised. However, it must be stated that especially in the lower part of the interval investigated from Hole 747A a biostratigraphic zonation was not feasible because of poor radiolarian preservation (Figs. 4 and 8).

In Hole 748B, most designations of the geomagnetic polarity intervals given in Schlich, Wise, et al. (1989) were changed considering the biostratigraphic results obtained in Hole $751 \mathrm{~A}$ and presented by Abelmann (1990). Except for the normal interval at the base of the sediment section studied, assigned to Subchron $6 \mathrm{C}$, the events were attributed to stratigraphically younger chrons (Figs. 5 and 8). Major disconformities were recognized in the upper and lower portions of the sediment interval investigated. A hiatus placed between Samples 120748B-8H-2, 45-47 cm, and -8H-1, 45-47 cm (59.1-57.6 mbsf) omits the early Miocene $C$. longithorax Zone and probably cuts Subchron 6A and part of Chron C6. The most prominent disconformity is at around $38.3 \mathrm{mbsf}$ and spans the time interval from $\sim 16.5$ to $\sim 11 \mathrm{Ma}$. This represents a slightly younger time range compared with the results of Schlich, Wise, et al. (1989), who estimated a time range from $\sim 17$ to $\sim 12 \mathrm{Ma}$ for a hiatus that was placed between Sections 120-748B-6H-2 and -6H-1 at a core depth of 39-40 mbsf. At this depth, however, there is a short hiatus that separates the $C$. golli regipileus and the $E$. punctatum zones and that omits, according to the diatom biostratigraphic study of Harwood and Mayurama (this volume), the Denticulopsis maccollumii diatom zone. A long-ranging hiatus, identified in this radiolarian study between 38.57 and 38.1 mbsf, includes three diatom zones that range in the upper portion of the middle Miocene (Harwood and Maruyama, this volume). Thus, it can be concluded that in Schlich, Wise, et al. (1989) the long-ranging middle Miocene hiatus was placed at the wrong core depth.

Also, in Hole 751A, many designations of the geomagnetic polarity intervals given in Schlich, Wise, et al. (1989) were changed, considering the biostratigraphic results presented by Abelmann (1990) (Figs. 6 and 8). Above the hiatus, which was placed between Sample 120-751A-13H-1, 63-69 cm, and Section 120-751A-12H-CC (109.7-109.2 mbsf) and which was interpreted to span a time interval between $\sim 14.0$ to $\sim 12.5$ $\mathrm{Ma}$, the designations remained unchanged. Below this middle Miocene hiatus, the designations were changed and the events were attributed to stratigraphically younger chrons (Figs. 6 and 8). Considering this stratigraphic reinterpretation, an age/depth diagram was constructed for the sediment sections studied in Hole 751A (Fig. 7). The average sedimentation rate derived for the early and middle Miocene is $8 \mathrm{~m} / \mathrm{m} . \mathrm{y}$.

Thyrsocyrtis clausa, a species originally described by Chen (1975), is restricted to the lower portion of the $E$. punctatum Zone (between Sections 120-751A-15H-CC and -13H-CC). This taxon was also recovered in DSDP Hole 266 (Leg 28), co-occurring with E. punctatum, according to the range chart prepared by Chen (1975, table 5). It should be noted that Chen erroneously placed the stratigraphic range of this species, which probably has some stratigraphic significance, into the Actinomma golownini Zone (Chen, 1975, table 2).

Based on the stratigraphic age assignment of the lower Miocene radiolarian zones proposed in this study, the age determination of the lower Miocene sediments recovered in Leg 113 Holes 689B and 690B was also revised (Fig. 8). The sequences corresponding to the $C$. longithorax and the $C$. antiqua zones were assigned to an older time interval in the early Miocene, ranging between approximately 19.5 and $22 \mathrm{Ma}$, compared with the age assignments proposed by Abelmann (1990) and Gersonde et al. (1990). But up to now the age determinations of the earliest Miocene interval are still tentative.

In all the holes investigated, distinct disconformities could be delineated, based on the absence of radiolarian and diatom zones and paleomagnetic polarity events in the obtained record. In Hole 748B, a lower Miocene hiatus was recognized, which omits the $C$. longithorax Zone and supposedly ranges from 22 to $19.5 \mathrm{Ma}$. In Holes $747 \mathrm{~A}$ and $751 \mathrm{~A}$, the sediment record probably also is disturbed by disconformities during and around the time interval corresponding to the $C$. longitho$\operatorname{rax}$ Zone. This assumption, however, is suggested only by the abundance and occurrence pattern of radiolarian species (e.g., in Core 120-751A-18H). Poor preservation (Hole 747A) and the lack of other stratigraphic data (Holes 747A and 751A) do not permit an unequivocal determination of these supposed hiatuses. High hiatus frequencies for the lower Miocene were also reported from the Leg 113 Sites 689 and 690 (Abelmann, 1990; Gersonde et al., 1990) (Fig. 8). 

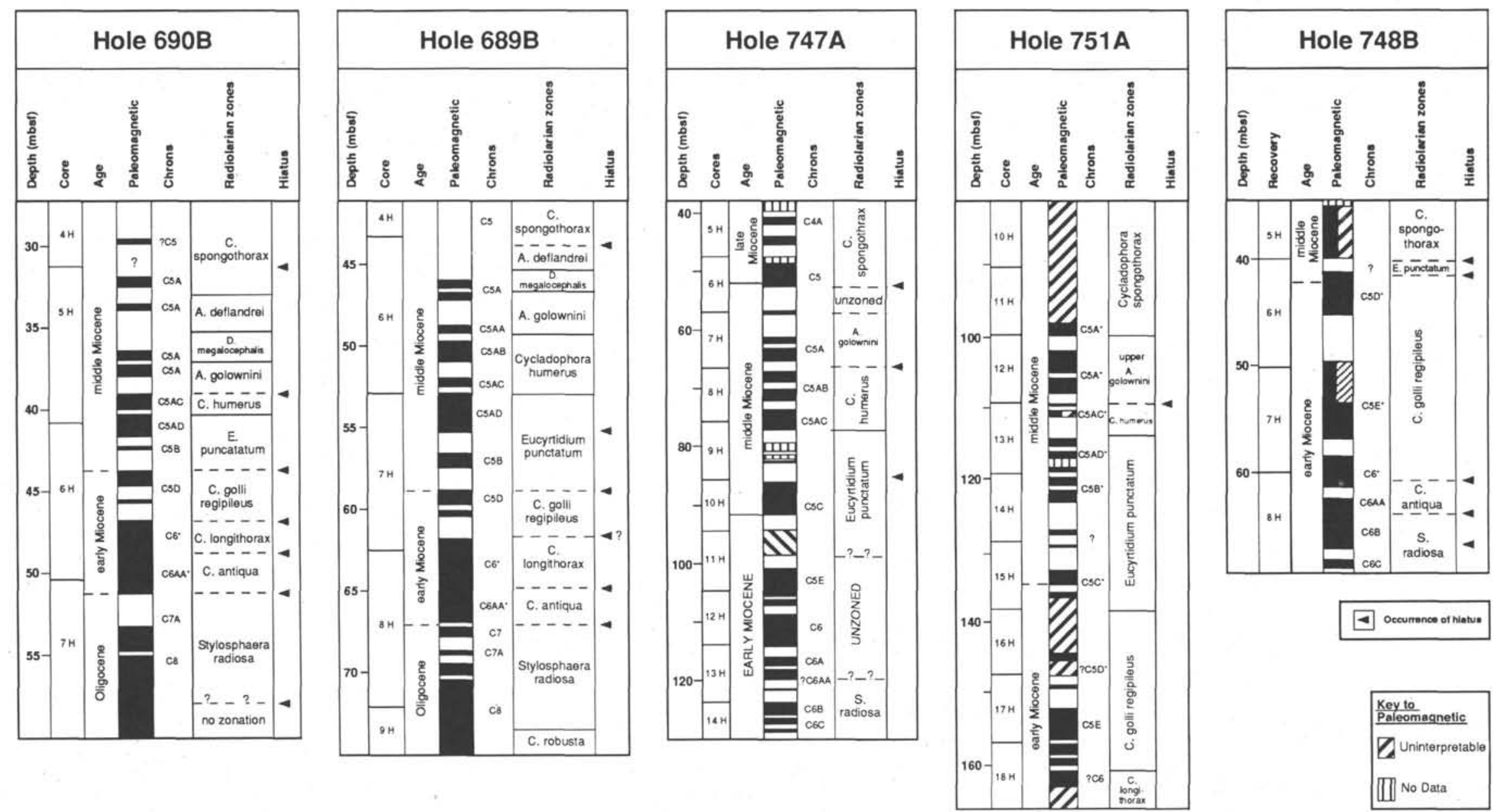

Figure 8. Comparison of radiolarian stratigraphies in the early and middle Miocene of Leg 113 Holes 689B and 690B (Abelmann, 1990) and Leg 120 Holes 747A, 748B, and 751A. Geomagnetic chron designations for the Leg 113 and 120 holes were taken from Gersonde et al. (1990) and Schlich, Wise, et al. (1989), respectively, except those marked by asterisks. Asterisk-marked chron designations indicate revisions based on the radiolarian biostratigraphic age determination. 


\section{A. ABELMANN}

Distinct disconformities in the middle Miocene interval occur in all of the holes investigated. The different age ranges of these disconformities were delineated. In Hole 747A, two disconformities occur during time intervals around $12.5 \mathrm{Ma}$ and 11-10 Ma, respectively, whereas in Hole 748 a longranging hiatus occurs between $\sim 16.5$ and $\sim 11$ Ma. A similar hiatus in Hole $751 \mathrm{~A}$ occurs between $\sim 14$ to $\sim 12.5 \mathrm{Ma}$. These disconformities can be linked to major climatic alterations between 16 and $13 \mathrm{Ma}$ that led to major glaciations on the Antarctic continent and cooling of bottom waters (Miller et al., 1987; Kennett and Hodell, 1986).

\section{CONCLUSION}

During Leg 120 lower to middle Miocene sediments recovered at Sites 747,748 , and 751 , which provide a detailed radiolarian stratigraphy. A zonation proposed by Abelmann (1990) for Leg 113 materials was used in this study, and its practicability tested. Modifications were made for upper middle Miocene zones. Two upper middle Miocene zones defined by Abelmann (1990), the Dendrospyris megalocephalis and Antarctissa deflandrei zones, were included in the Actinomma golownini Zone for reasons of practicability. The A. golownini Zone, originally defined by Chen (1975), was renamed and divided into an upper and a lower subzone by the FAD of $D$. megalocephalis.

The middle Miocene time interval, younger than $15 \mathrm{Ma}$, was dated according to the integrated biostratigraphic and geomagnetic results obtained by the study of Leg 113 sections. The age assignment of the radiolarian zonation for the lower Miocene proposed by Abelmann (1990) was improved and emended by this study because more expanded lower Miocene sequences were recovered in the Leg 120 holes. Most of the lower Miocene radiolarian zones could be correlated to an older time range compared with the age calibration proposed by Abelmann (1990). The improved and emended age calibration proposed in this study was used for a reinterpretation of the geomagnetic record and the stratigraphic age assignment of the lower Miocene sequences of Holes 689B and 690B (Leg 113). However, because the presence of disconformities and gaps in the geomagnetic record complicates an independent chronostratigraphic interpretation of these sediment intervals, some questions related to the accurate age determinations of lower Miocene radiolarian zones cannot yet be answered.

\section{SPECIES LIST}

Acrosphaera spp., Abelmann, 1990, p. 690, pl. 1, fig. 1.

Remarks. This species group includes all species of Acrosphaera. Actinomma golownini Petrushevskaya, 1975, p. 569, pl. 2, fig. 16; Abelmann, 1990, p. 691, pl. 1, fig. 8. (Plate 1, Figs. 7 and 8)

Actinomma holtedahli Björklund, 1976, p. 1121, pl. 20, figs. 8 and 9.

Actinomma medusa (Ehrenberg) Petrushevskaya, 1975, p. 568, pl. 2, figs. 6-8; Abelmann, 1990 , p. 690 , pl. 1, fig. 6.

Actinomma sp. A, Abelmann, 1990, p. 690, pl. 1, fig. 5. (Plate 1, Fig. 5)

Actinomma sp. B, Abelmann, 1990, p. 691, pl. 1, figs. 7A and 7B. (Plate 1, Figs. 3 and 4)

Amphistylus angelinus (Campbell and Clark) Chen, 1975, p. 453, pl. 21, figs. 3 and 4; Abelmann, 1990, p. 692, pl. 2, figs. 11A, 11B, and 11C.

Antarctissa deflandrei (Petrushevskaya) Lazarus 1990, p. 713, pl. 3, figs. 18-19; Abelmann, 1990, p. 694, pl. 4, figs. 10A and 10B.

Antarctissa robusta Petrushevskaya, 1975, p. 591, pl. 11, figs. 21 and 22; Abelmann, 1990 , p. 694 , pl. 4 , fig. 9.

Anthocyrtidium sp. (Plate 3, Figs. 3 and 4)

Artophormis gracilis Riedel, 1959, p. 300, pl. 2, figs. 12 and 13; Sanfilippo et al., 1973, pl. 4, fig. 4.

Calocyclas cf. semipolita Clark and Campbell, 1942, p. 83, pl. 8, figs. 12, 14, 17-19, and 21-23; Abelmann, 1990, p. 697, pl. 7, fig. 4. (Plate 5, Fig. 8)

Carpocanarium papillosum (Ehr.) Nigrini and Moore, 1979, p. N27, pl. 21 , fig. 3.
Cenosphaera sp. A, Abelmann, 1990, p. 691, pl. 3, fig. 12. (Plate 1, Fig. 6)

Ceratocyrtis mashae, Björklund, 1976, p. 1125, pl. 17, figs. 1-8; Abelmann, 1990, p. 694, pl. 4, figs. 15A, 15B, and 15C.

Ceratocyrtis stigi (Björklund) Nigrini and Lombari, 1984, p. N13, pl. 15, fig. 7; Abelmann, 1990, p. 694, pl. 4, fig. 12.

Circodiscus ellipticus (Stöhr) group, Petrushevskaya, 1975, p. 575, pl. 6, figs. 1-6; Abelmann, 1990, p. 693, pl. 3, fig. 8.

Collosphaerid sp. B, Abelmann, 1990, p. 690, pl. 1, fig. 11.

Cornutella clathrata Ehrenberg, 1838, p. 129; 1844, p. 77; 1847b, p. 42, 1854b, pl. 22, figs. 39a-39c; Abelmann, 1990, p. 696, pl. 8, fig. 8 .

Cornutella profunda Ehrenberg, 1858, p. 31; Riedel, 1958, p. 232, pl. 3 , figs. 1 and 2 .

Corythomelissa horrida Petrushevskaya, 1975, p. 590, pl. 11 , figs. 14 and 15, pl. 21, fig. 9; Abelmann, 1990, p. 697, pl. 7, fig. 9.

Corythospyris fiscella Goll, 1978, p. 178, pl. 5, figs. 1-21; Abelmann, 1990, p. 695, pl. 5, figs. 4A, 4B, and 7. (Plate 4, Fig. 9)

Cycladophora antiqua Abelmann, 1990, p. 698, pl. 7, figs. 13A and 13B.

Cycladophora conica Lombari and Lazarus, 1988, p. 105, pl. 3, figs. $1-16$.

Cycladophora golli golli (Chen) Lombari and Lazarus, 1988, p. 124, pl. 11 , figs. 3 and 4 .

Cycladophora golli regipileus (Chen) Lombari and Lazarus, 1988, p. 124 , pl. 11, figs. 6,8 , and 9 .

Cycladophora humerus (Petrushevskaya) Lombari and Lazarus, 1988, p. 123, pl. 9, figs. 1-6.

Cycladophora sp. (Plate 5, Fig. 9)

Cycladophora spongothorax (Chen) Lombari and Lazarus, 1988, p. 122 , pl. 8, figs. 1-6.

Cyrtocapsella cornuta (Haeckel) Sanfilippo and Riedel, 1970, p. 453, pl. 1, figs. 19-20.

Cyrtocapsella longithorax (Petrushevskaya) Abelmann, 1990, p. 696, pl. 5, figs. $12 \mathrm{~A}$ and $12 \mathrm{~B}$.

Cyrtocapsella robusta Abelmann, 1990, p. 696, pl. 5, figs. 10 and 11. (Plate 5, Fig. 7)

Cyrtocapsella tetrapera (Haeckel) Sanfilippo and Riedel, 1970, p. 453, pl. 1, figs. 1-3; Nigrini and Lombari, 1984, pp. N109-N110, pl. 23, fig. 5 .

Dendrospyris megalocephalis Chen, 1975, p. 455, pl. 14, fig. 3-5; Abelmann, 1990, p. 695 , pl. 5, fig. 15. (Plate 5, Fig. 10)

Dendrospyris rhodospyroides (Petrushevskaya) Abelmann, 1990, p. 695 , pl. 5, figs. 9A and 9B.

Dendrospyris stabilis Goll, 1968, pp. 1422-1423, pl. 173, figs. 16-18; Abelmann, 1990, p. 695, pl. 5, figs. 8A and 8B.

Dictyophimus gracilipes Bailey, 1856, p. 4, pl. 1, fig. 8; Petrushevskaya, 1967 , p. 65 , figs. 38 and 39.

Disolenia spp. group, Abelmann, 1990, p. 690, pl. 1, figs. 3A and 3B.

Druppatractus hastatus Blueford, 1982, p. 206, pl. 6, figs. 3-4; Lazarus and Pallant, 1988, p. 42, pl. 6, figs. 13-15; Abelmann, 1990 , p. 691 , pl. 2 , fig. 3.

Eucyrtidium calvertense Martin group, Abelmann, 1990, p. 696, pl. 6, figs. 4 and 5A-5C. (Plate 4, Fig. 6)

Eucyrtidium cienkowskii Haeckel group, Sanfilippo et al., 1973, p. 221 , pl. 5, figs. 7-11; Abelmann, 1990, p. 696, pl. 6, figs. 3A-3D. (Plate 4, Figs. 1 and 2)

Eucyrtidium punctatum (Ehrenberg) Ehrenberg, 1847b, p. 43; 1854, pl. 22, fig. 24; Abelmann, 1990, p. 696, pl. 6, fig. 6. (Plate 4, Figs. 3 and 4)

Eucyrtidium sp. A (Plate 4, Fig. 7)

Eucyrtidium sp. B (Plate 4, Fig. 8)

Gondwanaria deflandrei Petrushevskaya, 1975, p. 584, pl. 9, figs. 8 and 9; Abelmann, 1990, p. 697, pl. 7, fig. 7.

Gondwanaria japonica (Nakaseko) group, Petrushevskaya, 1975, p. 584, pl. 8, fig. 15, pl. 9, figs. 2-7, pl. 12, fig. 1; Abelmann, 1990, p. 697 , pl. 7 , figs. $3 \mathrm{~A}$ and $3 \mathrm{~B}$.

Gondwanaria sp. A, Abelmann, 1990, p. 697, pl. 7, fig. 6 .

Heliodiscus sp. A, Abelmann, 1990 , p. 692, pl. 3, figs. 2 and 3.

Heliodiscus(?) sp. B. (Plate 1, Figs. 1 and 2)

Hexacontium enthacanthum Jörgensen, 1900, p. 52, pl. 2, fig. 14, pl. 4 , fig. 20; Benson, 1966, p. 149 , pl. 3 , figs. 13 and 14 , pl. 4 , figs. 1-3; Nigrini and Moore, 1979, p. S45, pl. 5, figs. 1a and 1b.

Hexastylus spp. Nigrini and Lombari, 1984, p. S17, pl. 3, figs. 1a-1c; Abelmann, 1990, p. 691, pl. 1, fig. 10. 
Lamprocyrtis (?) hannai (Campbell and Clark) Nigrini and Lombari, 1984, p. N165, pl. 30, figs. 2a-2b. (Plate 3, Figs. 5 and 6)

Lamprocyclas sp. group, Kling, 1973, p. 731, pl. 13, fig. 2; Weaver and Dinkelmann, 1978 , p. 869, pl. 2, figs. 7 and 8. (Plate 3, Fig. 7)

Lithocarpium polyacantha (Campbell and Clark) group, Petrushevskaya, 1975, p. 572, pl. 3, figs. 6-8 (only); Abelmann, 1990, p. 694, pl. 4 , fig. 4 .

Lithomelissa cf. ehrenbergi Bütschlii sensu Chen, Abelmann, 1990, p. 695 , pl. 4 , fig. 13.

Lithomelissa robusta Chen, 1975 , p. 457 , pl. 9, figs. 1 and 2; Abelmann, 1990, p. 695, pl. 5, figs. 2A and 2B.

Lychnocanoma conica (Clark and Campbell) Abelmann, 1990, p. 697 , pl. 6 , fig. 8, pl. 7, figs. $1 \mathrm{~A}$ and $1 \mathrm{~B}$.

Lychnocanoma sp. B, Abelmann, 1990, p. 697, pl. 7, figs. 2A, 2B. (Plate 5, Fig. 4)

Lychnocanoma sp. C (Plate 5, Figs. 1 and 2)

Prunopyle hayesi Chen, 1975, p. 454, pl. 9, figs. 3-5; Abelmann, 1990, p. 693 , pl. 3, fig. 14 .

Prunopyle tetrapila Hays, 1965, p. 172, pl. 2, fig. 5; Abelmann, 1990, p. 693 , pl. 3, fig. 13.

Prunopyle titan Campbell and Clark, 1944, p. 20, pl. 3, figs. 1-3; Hays, 1965, p. 173, pl. 2, fig. 4; Weaver, 1976, p. 578, fig. 6; Abelmann, 1990 , p. 693 , pl. 3 , fig. 16.

Prunopyle sp. A, Abelmann, 1990, p. 693, pl. 3, fig. 15.

Prunopyle sp. B, Abelmann, 1990, p. 693, pl. 4, figs. 3A and 3B.

Prunopyle sp. D, Abelmann, 1990, p. 693, pl. 4, figs. 1A and 1B.

Pterocanium sp. (Plate 5, Fig. 3)

Rhopalodictyum sp. (Plate 3, Fig. 11)

Saccospyris antarctica Petrushevskaya, 1975, p. 589, pl. 13, figs. 19 and 20.

Siphocampe arachnea (Ehrenberg) group, Nigrini, 1977, p. 255, pl. 3, figs. 7 and 8.

Spongodiscus craticulatus (Stöhr) Petrushevskaya, 1975, p. 574, pl. 5, figs. 9 and 10; Abelmann, 1990, p. 692, pl. 3, fig. 7.

Spongomelissa dilli Chen, 1975, p. 458, pl. 10, fig. 4. (Plate 5, Figs. 5 and 6)

Spongopyle osculosa Dreyer, 1889, p. 42, pl. 11, figs. 99 and 100; Riedel, 1958, p. 226, pl. 1, fig. 12; Nigrini and Moore, 1979, p. S115, pl. 15, fig. 1; Nigrini and Lombari, 1984, p. S77, pl. 11, figs. 1a and 1b.

Spongotrochus glacialis Popofsky group, Petrushevskaya, 1975, p. 575 , pl. 5, fig. 8, pl. 35, figs. 1-6 (with synonymy); Nigrini and Moore, 1979, p. S117, pl. 15, figs. 2a-2d; Nigrini and Lombari, 1985 , p. S79, pl. 11, fig. 2.

Spongotrochus spp. Abelmann, 1990, p. 693, pl. 3, figs. 17A and 17B.

Stauroxiphos communis Carnevale, 1908, p. 15, pl. 2, fig. 9; Abelmann, 1990 , p. 692, pl. 2, fig. 12 (with synonymy). (Plate 2, Figs. 2 and 3)

Stichophormis sp. Chen 1975, p. 462, pl. 13, fig. 8; Abelmann, 1990, p. 698 , pl. 8, fig. 9. (Plate 4, Fig. 5)

Stylatractus neptunus Haeckel, 1887, p. 328, pl. 17, fig. 6; Riedel, 1958, p. 226, pl. 1, fig. 9; Abelmann, 1990, p. 691, pl. 2, figs. 8 and 9.

Stylatractus santaeannae (Campbell and Clark) Petrushevskaya and Kozlova, 1972, p. 520, pl. 11, fig. 10; Abelmann, 1990, p. 691, pl. 2, fig. 6.

Stylodictya validispina Jörgensen, 1905, p. 119, pl. 10, fig. 40; Petrushevskaya, 1967, p. 33, fig. 17IV-17V; Nigrini and Moore, 1979, p. S103, pl. 13, figs. 5a and 5b; Nigrini and Lombari, 1985, p. S71, pl. 10, fig. 2.

Stylosphaera coronata laevis (Ehrenberg) Sanfilippo and Riedel, 1973 , p. 520, pl. 1, fig. 19; pl. 25, figs. 5 and 6; Abelmann, 1990, p. 692 , pl. 2 , fig. 5 .

Stylosphaera radiosa Ehrenberg group, Abelmann, 1990, p. 692, pl. 2, figs. $4 \mathrm{~A}-4 \mathrm{C}$ and 7 . (Plate 2, Figs. 4 and 5)

Stylosphaera sp. A, Abelmann, 1990, p. 692, pl. 2, fig. 10. (Plate 2, Figs. 6 and 7)

Stylosphaera sp. B (Plate 2, Fig. 1)

Stylosphaera sp. C (Plate 2, Figs. 8 and 9)

Tholoniid sp. B, Abelmann, 1990, p. 694, pl. 4, fig. 8.

Thyrsocyrtis clausa Chen, 1975, p. 463, pl. 14, figs. 1 and 2. (Plate 3, Figs. 1 and 2)

Tripilidium(?) clavipes advena, Clark and Campbell, 1945, p. 34, pl. 7, figs. 31-33; Abelmann, 1990, p. 695, pl. 5, fig. 1.

Trissocyclid sp. A, Abelmann, 1990, p. 695, pl. 5, figs. 5A-5C.

Velicucullus altus Abelmann, 1990, p. 698, pl. 8, figs. 5A-5C. (Plate 3, Fig. 8)

Velicucullus cf. oddgurneri (Björklund), Abelmann, 1990, p. 698, pl. 8, fig. 6. (Plate 3, Figs. 9 and 10)

\section{ACKNOWLEDGMENTS}

I am grateful to C. A. Nigrini for his critical review of this manuscript. Ruth Cordelair and Inge Klappstein are thanked for laboratory and technical assistance. I wish to thank R. Gersonde for fruitful discussions and his critical reading of the manuscript. W. A. Berggren and D. M. Harwood kindly provided stratigraphic information. D. Lazarus is thanked for making available the sample set studied. This is AlfredWegener-Institute Contribution No. 288.

\section{REFERENCES}

Abelmann, A., 1989. Freeze-drying simplifies the preparation of microfossils. Micropaleontology, 34:371.

1990. Oligocene to middle Miocene radiolarian stratigraphy of southern high latitudes from Leg 113, Sites 689 and 690. In Barker, P. F., Kennett, J. P., et al., Proc. ODP, Sci. Results, 113: College Station, TX (Ocean Drilling Program).

Bailey, J. W., 1856. Notice of microscopic forms found in the soundings of the Sea of Kamtschatka. Am. J. Sci. Arts, 2:1-6.

Baldauf, J. A., and Barron, J. A., 1991. Diatom biostratigraphy: Kerguelen Plateau and Prydz Bay regions of the Southern Oceans. In Barron, J. A., Larsen, B., et al., Proc. ODP, Sci. Results, 119: College Station, TX (Ocean Drilling Program).

Barker, P. F., Kennett, J. P., et al., 1988. Proc. ODP, Init. Repts., 113: College Station, TX (Ocean Drilling Program).

Barron, J. A., 1985. Miocene to Holocene planktic diatoms. In Bolli, H. M., Saunders, J. B., and Perch-Nielsen, K. (Eds.), Plankton Stratigraphy: Cambridge (Cambridge Univ. Press), 763-809.

Barron, J. A., Nigrini, C. A., Pujos, A., Saito, T., Theyer, F., Thomas, E., and Weinreich, E., 1985. Synthesis of biostratigraphy, central equatorial Pacific, Deep Sea Drilling Project Leg 85: refinement of Oligocene to Quarternary biochronology. In Meyer, L., Theyer, F., Thomas, E., et al., Init. Repts. DSDP, 85: Washington (U.S. Govt. Printing Office), 905-934.

Barron, J. A., Baldauf, J. G., Barrera, E., Caulet, J.-P., Huber, B. T., Keating, B. H., Lazarus, D., Sahai, H. Thierstein, H. R., and Wei, W., 1991. Biochronologic and magnetochronologic synthesis of Leg 119 sediments from the Kerguelen Plateau and Prydz Bay, Antarctica. In Barron, J. A., Larsen, B., et al., Proc. ODP, Sci. Results, 119: College Station, TX (Ocean Drilling Program).

Basov, I. A, Ciesielski, P. F., Krasheninnikov, V. A., Weaver, F. M., and Wise, S. W., 1983. Biostratigraphic and paleontologic synthesis: Deep Sea Drilling Project Leg 71, Falkland Plateau and Argentine Basin. In Ludwig, W. J., Krasheninnikov, V. A., et al., Init. Repts. DSDP, 71, Pt. 1: Washington (U. S. Govt. Printing Office), 445-460.

Benson, R. N., 1966. Recent Radiolaria from the Gulf of California [Ph.D. dissert.]. Minnesota Univ.

Berggren, W. A., Kent, D. V., and Van Couvering, J. A., 1985. The Neogene: Part 2. Neogene geochronology and chronostratigraphy. In Snelling, N. J. (Ed.), The Chronology of the Geological Record. Mem. Geol. Soc. (London), 10:211-260.

Björklund, K. R., 1976. Radiolaria from the Norwegian Sea, Leg 38 of the Deep Sea Drilling Project. In Talwani, M., Udintsev, G., et al., Init. Repts. DSDP, 38: Washington (U.S. Govt. Printing Office), 1101-1168.

Blueford, J. R., 1982. Miocene actinommid Radiolaria from the equatorial Pacific. Micropaleontology, 28:189-213.

Campbell, A. S., and Clark, B. L., 1944. Miocene radiolarian faunas from southern California. Spec. Pap., Geol. Soc. Am., 51:1-77.

Carnevale, P., 1908. Radiolarie e sillicoflagellati di Bergonzano (Reggio Emilia). Veneto Sci., Lett. Arti, Mem., 28:1-46.

Chen, P.-H., 1975. Antarctic Radiolaria. In Hayes, D. E., Frakes, L. A., et al., Init. Repts. DSDP, 28: Washington (U.S. Govt. Printing Office), 437-514.

Clark, B. L., and Campbell, A. S., 1942. Eocene radiolarian faunas from the Mt. Diablo area, California. Spec. Pap., Geol. Soc. Am., 39:1112 .

1945. Radiolaria from the Kreyhagen Formation, near Los Banos, California. Mem. Geol. Soc. Am., 10:1-66 (i-vii).

Dreyer, F., 1889. Morphologische Radiolarienstudien. 1. Die Pylombildungen in vergleichend-anatomischer und entwicklungsgeschichtlicher Beziehung bei Radiolarien und Protisten überhaupt 
nebst System und Beschreibung neuer und der bis jetzt bekannten pylomatischen Spumellarien. Jena. Z. Med. Naturwiss., 23:1-138.

Ehrenberg, C. G., 1838. Über die Bildung der Kreidefelsen und des Kreidemergels durch unsichtbare Organismen. Abh. Kgl. Akad. Wiss. Berlin, 59-147.

1844. Über zwei neue Lager von Gebirgsmassen aus Infusorien als Meeres-Absatz in Nord-Amerika und eine Vergleichung derselben mit den organischen Kreidegebilden in Europa und Afrika. Kgl. Preuss. Akad. Wiss. Berlin, 57-97.

1847a. Über eine halibiolithische, von Herrn Schomburgk entdeckte, vorherrschend aus mikroskopischen Polycystinen gebildete Gebirgsmasse von Barbados. Monatsber. Kgl. Preuss. Akad. Wiss. Berlin, 382-385.

1847b. Über die mikroskopischen kieselschaligen Polycystinen als mächtige Gebirgsmasse von Barbados und über das Verhältnis der aus mehr als 300 neuen Arten bestehenden ganz eigentümlichen Formengruppe jener Felsmasse zu den jetzt lebenden Tieren und zur Kreidebildung. Eine neue Anregung zur Erforschung des Erdenlebens. Monatsber. Kgl. Preuss. Akad. Wiss. Berlin, 40-60.

1854a. Die systematische Charakteristik der neuen mikroskopischen Organismen des tiefen atlantischen Ozeans. Monatsber. Kgl. Preuss. Akad. Wiss. Berlin, 236-250.

1854b. Mikrogeologie, 27:1-374, ATLAS, 1-31, 40 pls., Fortsetzung (1856): Leipzig (Voss).

1858. Kurze Characteristik der 9 neuen Genera und der 105 neuen Species des ägäischen Meeres und des Tiefgrundes des MittelMeeres. Monatsber. Kgl. Preuss. Akad. Wiss. Berlin, 10-40.

Gersonde, R., and Burckle, L. H., 1990. Neogene diatom biostratigraphy of ODP Leg 113, Weddell Sea (Antarctic Ocean). In Barker, P. F., Kennett, J. P., et al., Proc. ODP, Sci. Results, 113: College Station, TX (Ocean Drilling Program), 761-789.

Gersonde, R., Abelmann, A. Burckle, L. H., Hamilton, N., Lazarus, D., McCartney, K., O'Brien, P., SpieB, V., and Wise, S. W., Jr., 1990. Biostratigraphic synthesis of Neogene siliceous microfossils from the Antarctic Ocean, ODP Leg 113 (Weddell Sea). In Barker, P. F., Kennett, J. P., et al., Proc. ODP, Sci. Results, 113: College Station, TX (Ocean Drilling Program), 915-936.

Goll, R. M., 1968. Classification and phylogeny of Cenozoic Trissocyclidae (Radiolaria) in the Pacific and Caribbean basins (Pt. I). J . Paleontol., 42:1409-1432.

1978. Five trissocyclid Radiolaria from Site 38. In Talwani, M., Udintsev, G., et al., Init. Repts. DSDP, Suppl. to Vols. 38, 39, 40, and 41: Washington (U.S. Govt. Printing Office), 177-191.

Haeckel, E., 1887. Report on the Radiolaria collected by H.M.S. Challenger during the years 1873-1876. Rep. Sci. Results, H.M.S. Challenger, 1873-1876. Zoology, 18, 2 parts, clxxxviii +1803 pp. atlas.

Hayes, D. E., and Frakes, L. A., 1975. General synthesis, Deep Sea Drilling Project Leg 28. In Hayes, D. E., Frakes, L. A., et al., Init. Repts. DSDP, 28: Washington (U.S. Govt. Printing Office), 919-942.

Hays, J. D., 1965. Radiolaria and late Tertiary and Quarternary history of Antarctic Seas. In Llano, G. A. (Ed.), Biology of Antarctic Seas II. Am. Geophys. Union Antarct. R. Ser., 5:125-184.

Hays, J. D., and Opdyke, N. D., 1967. Antarctic Radiolaria, magnetic reversals and climatic change. Science, 158:1001-1011.

Jörgensen, E., 1900. Protophyten und Protozoen im Plankton der Norwegischen Westküste. Bergens Museums Aarbog, 2:51-95.

1905. The protist plankton and the diatoms in bottom samples. Bergens Museums Skrifter, 7:49-151.

Keany, J., 1979. Early Pliocene radiolarian taxonomy and biostratigraphy in the Antarctic region. Micropaleontology, 25:50-74.

Kennett, J. P., and Hodell, D. A., 1986. Major events in Neogene oxygen isotopic records. South African J. Sci., 82:497-498.

Kling, S. A., 1973. Radiolaria from the eastern North Pacific, Deep Sea Drilling Project, Leg 18. In Kulm, L. D., von Huene, R., et al., Init. Repts. DSDP, 18: Washington (U.S. Govt. Printing Office), 155-182.

Lazarus, D., 1990. Middle Miocene to recent radiolarians from the Weddell Sea, Antarctica, ODP Leg 113. In Barker, P. F., Kennett, J. P., et al., Proc. ODP, Sci. Results, 113: College Station, TX (Ocean Drilling Program), 709-727.

Lazarus, D., and Pallant, A., 1988. Oligocene and Neogene Radiolaria from the Labrador Sea, ODP Leg 105. In Arthur, M. A., Srivas- tava, S. P., et al., Proc. ODP, Sci. Results, 105: College Station, TX (Ocean Drilling Program), 349-380.

Lombari, G., and Lazarus, D., 1988. Neogene cycladophorid radiolarians from North Atlantic, Antarctic, and North Pacific deep-sea sediments. Micropaleontology, 35:97-135.

Miller, K. G., Fairbanks, R. G., and Mountain, G. S., 1987. Tertiary oxygen isotope synthesis, sea level history, and continental margin erosion. Paleoceanography, 2:1-19.

Nigrini, C., 1977. Tropical Cenozoic Artostrobiidae (Radiolaria). Micropaleontology, 23: 241-269.

Nigrini, C., and Lombari, G., 1984. A guide to Miocene Radiolaria. Spec. Publ., Cushman Found. Foraminiferal Res., No. 22.

Nigrini, C., and Moore, T. C., Jr., 1979. A guide to modern Radiolaria. Spec. Publ., Cushman Found. Foraminiferal Res., No. 16.

Petrushevskaya, M. G., 1967. Radiolarians of order Spumellaria and Nassellaria of the Antarctic Region. In Andriiashev, A. P., and Ushakov, P. V. ( Eds.), Biological Report of the Soviet Antarctic Expedition, 1955-1958, Jerusalem (Israel Progr. Sci. Trans.), 3:1186.

1975. Cenozoic radiolarians of the Antarctic, Leg 29, Deep Sea Drilling Project. In Kennett, J. P., Houtz, R. E., et al., Init. Repts. DSDP, 29: Washington (U.S. Govt. Printing Office), 541-676.

Petrushevskaya, M. G., and Kozlova, G. E., 1972. Radiolaria: Leg 14, Deep Sea Drilling Project. In Hayes, D. E., Pimm, A. C., et al., Init. Repts. DSDP, 14: Washington (U.S. Govt. Printing Office), 495-648.

Riedel, W. R., 1958. Radiolaria in Antarctic sediments. Repts. B.A.N.Z. Antarct. Res, Exped., B-6(10):218-254.

1959. Oligocene and lower Miocene Radiolaria in tropical Pacific sediments. Micropaleontology, 5:285-302.

Riedel, W. R., and Sanfilippo, A., 1970. Radiolaria, Leg 4, Deep Sea Drilling Project. In Bader, R. G., Gerard, R. D., et al., Init. Repts. DSDP, 4: Washington (U.S. Govt. Printing Office), 503-575. 1978. Stratigraphy and evolution of tropical Cenocoic radiolarians. Micropaleontology, 23:61-96.

Sanfilippo, A., Burckle, L. H., Martini, E., and Riedel, W. R., 1973. Radiolarians, diatoms, silicoflagellates and calcareous nannofossils in the Mediterranean Neogene. Micropaleontology, 19:209-234.

Sanfilippo, A., and Riedel, W. R., 1970. Post-Eocene "closed" theoperid radiolarians. Micropaleontology, 16:446-462.

1973. Cenozoic Radiolaria (exclusive of Theoperids, Artostrobiids and Amphipyndacids) from the Gulf of Mexico, Deep Sea Drilling Project Leg 10. In Worzel, J. L., Bryant, W., et al., Init. Repts. DSDP, 10: Washington (U.S. Govt. Printing Office), 457-611.

Schlich, R., Wise, S. W., Jr., et al., 1989. Proc. ODP, Init. Repts, 120: College Station, TX (Ocean Drilling Program).

Spieß, V., 1990. Cenocoic magnetostratigraphy of ODP Leg 113 drill sites on Maud Rise, Weddell Sea, Antarctica. In Barker, P. F., Kennett, J. P., et al., Proc. ODP, Sci. Results, 113: College Station, TX (Ocean Drilling Program), 915 936.

Tauxe, L., Petersen, N. P., and LaBreque, J. L., 1984. Magnetostratigraphy of Leg 73 sediments. In Hsü, K. L., LaBreque, J. L., et al., Init. Repts. DSDP, 73: Washington (U.S. Govt. Printing Office), 609-621.

Weaver, F. M., 1976. Antarctic Radiolaria from the southeast Pacific Basin, DSDP Leg 35. In Hollister, C. D., Craddock, C., et al., Init. Repts. DSDP, 35: Washington (U.S. Govt. Printing Office), 569-603.

1983. Cenozoic radiolarians from the southwest Atlantic, Falkland Plateau Region, Deep Sea Drilling Project Leg 71. In Ludwig, W. J., Krasheninnikov, V. A., et al., Init. Repts. DSDP, 71, Pt. 2: Washington (U. S. Govt. Printing Office), 667-686.

Weaver, F. M., and Dinkelman, M. G., 1978. Cenozoic radiolarians from the Blake Plateau and the Blake-Bahama Basin, DSDP Leg 44. In Benson, W. E., Sheridan, R. E., et al., Init. Repts. DSDP, 44: Washington (U. S. Govt. Printing Office), 865-886.

Weaver, F. M., Rögl, F., Haq, B. U., and Schrader, H.-J., 1976. Paleontological summary of Deep Sea Drilling Program results from Leg 35, Southeast Pacific Basin. In Hollister, C. D., Craddock, C., et al., Init. Repts. DSDP, 35: Washington (U. S. Govt. Printing Office), 531-537.

Date of initial receipt: 13 February 1990

Date of acceptance: 13 November 1990

Ms 120B-165 


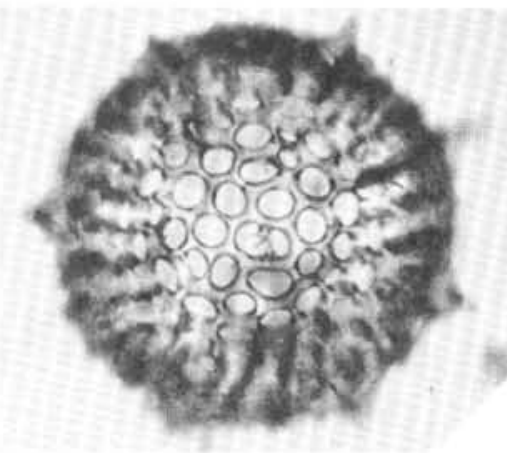

1

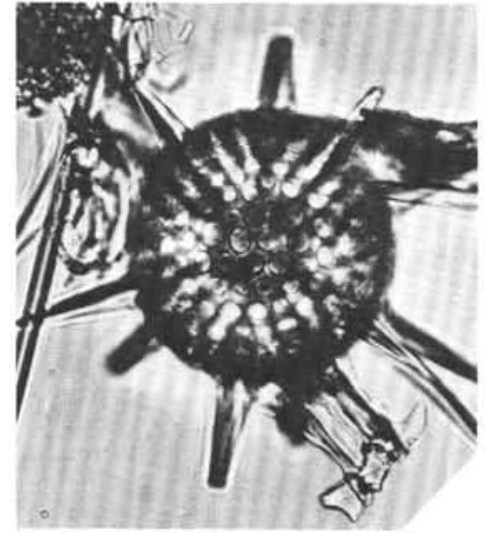

5

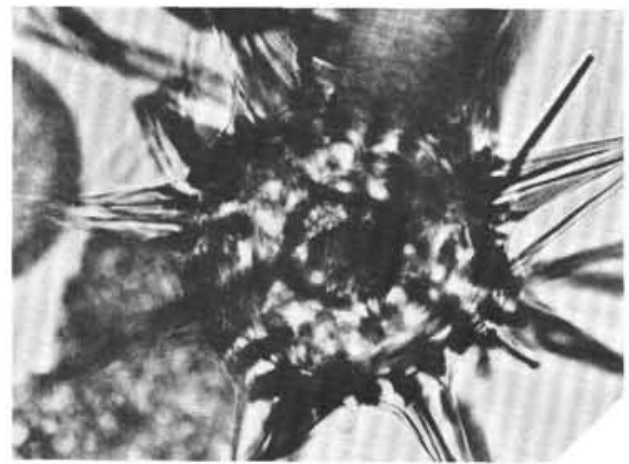

7

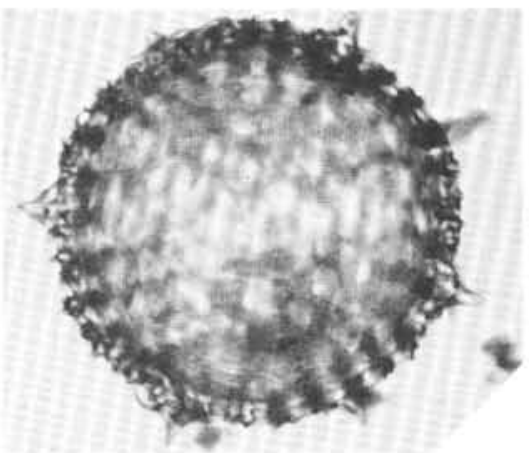

2

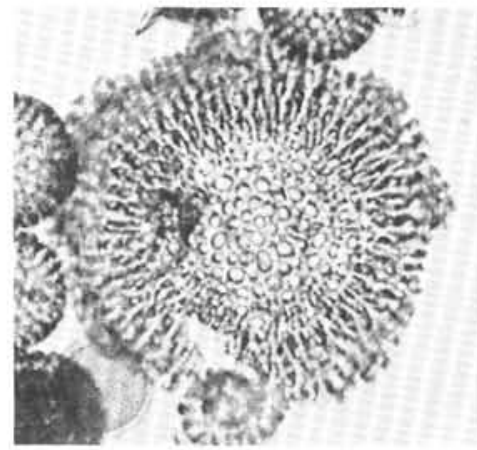

6

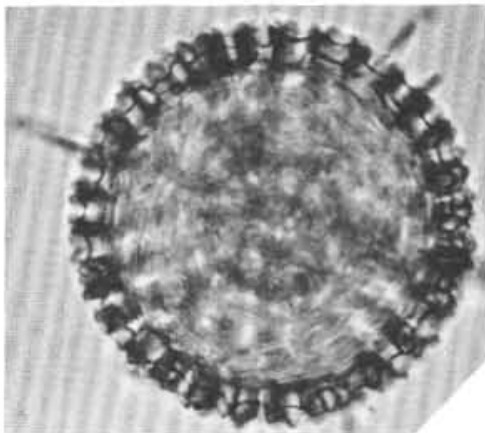

3

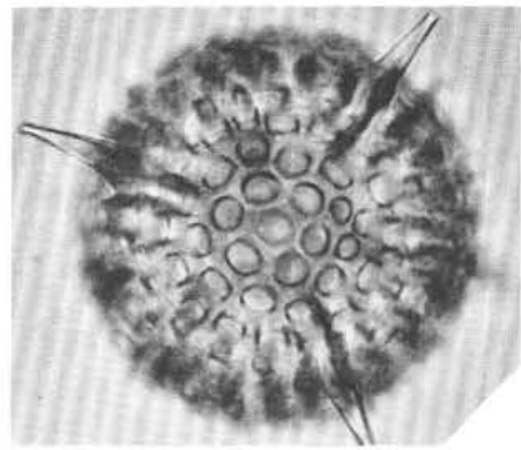

4

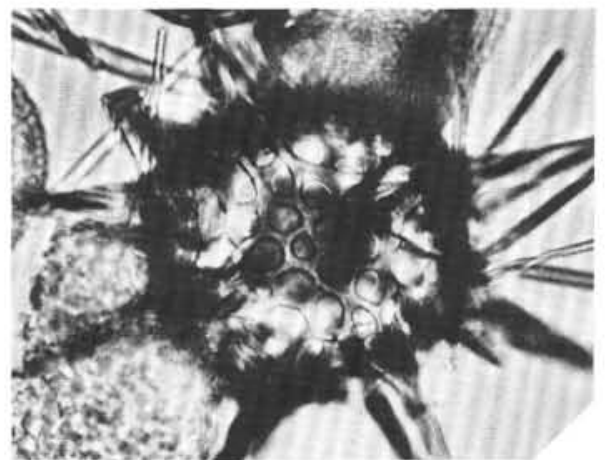

8

Plate 1. 1, 2. Heliodiscus(?) sp. B, $\times 177$, Sample 120-747A-13H-CC. 3, 4. Actinomma sp. B, $\times 293$, Sample $120-747$ A-8H-7, 45-47 cm 5. Actinomma sp. A, $\times 193$, Sample $120-751$ A-17H-2, $98-100 \mathrm{~cm}$. 6. Cenosphaera sp. A, $\times 112$, Sample $120-748 \mathrm{~B}-8 \mathrm{H}-6,45-47 \mathrm{~cm}$. 7, 8. Actinomma golownini, $\times 193$, Sample 120-751A-10H-CC. 


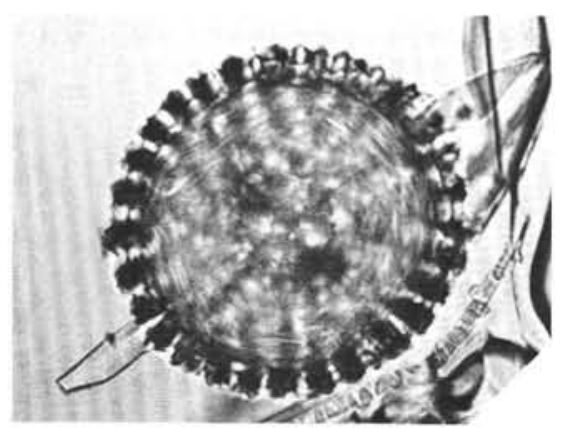

1
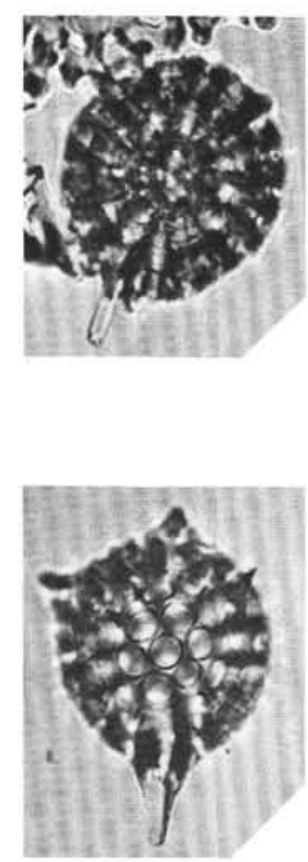

5

6

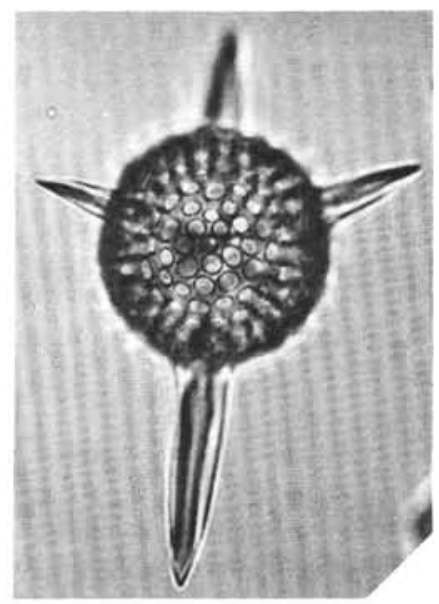

2
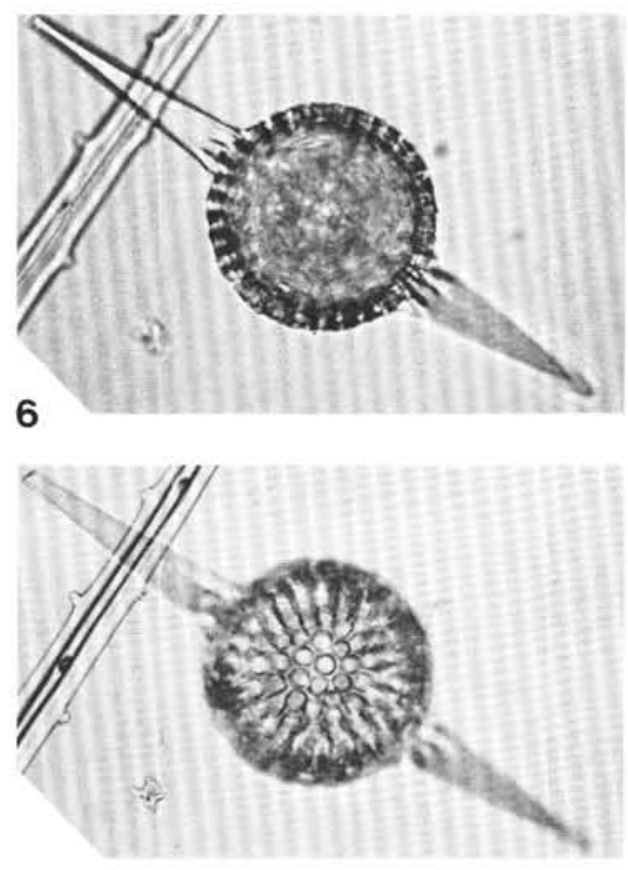

7

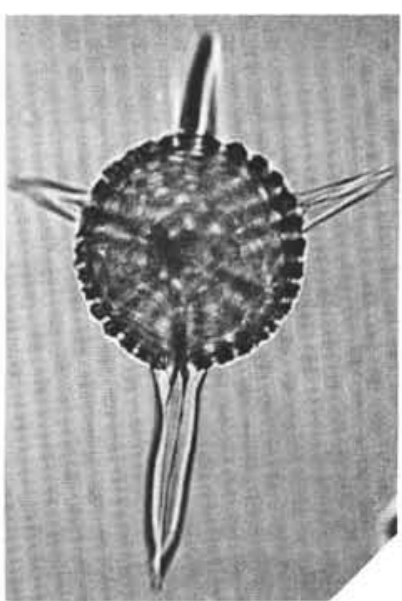

3

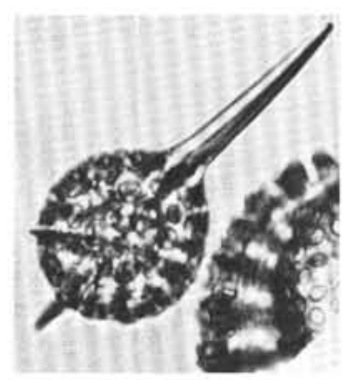

8

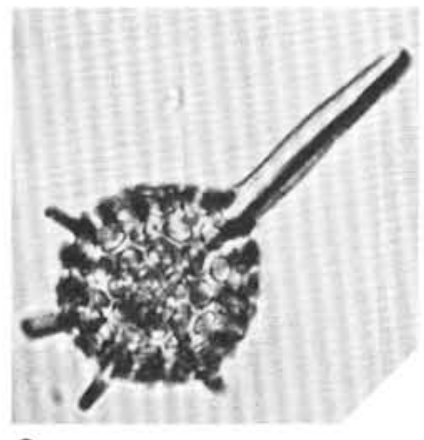

9

Plate 2. 1. Stylosphaera sp. B, $\times 293$, Sample 120-747A-10H-CC. 2, 3. Stauroxiphos communis, $\times 293$, Sample 120-747A-8H-CC. 4, 5 . Stylosphaera radiosa, $\times 293$; (4) Sample 120-748B-8H-CC, (5) Sample 120-747A-13H-CC. 6, 7. Stylosphaera sp. A, $\times 293$, Sample 120-747A-9H-8, 45-47 cm. 8, 9. Stylosphaera sp. C, $\times 293$, Sample 120-748B-8H-2, $45-47 \mathrm{~cm}$. 


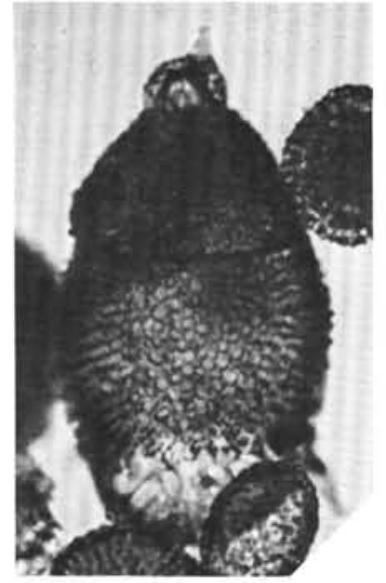

1

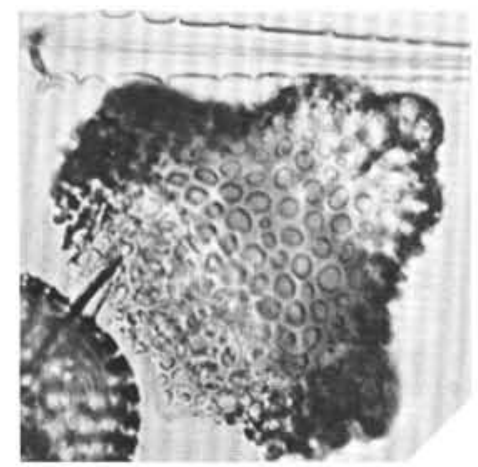

8

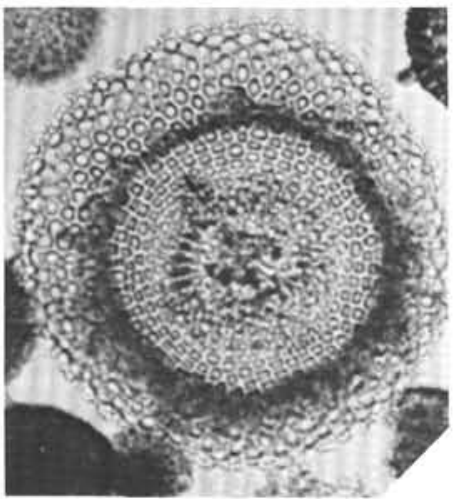

9

2
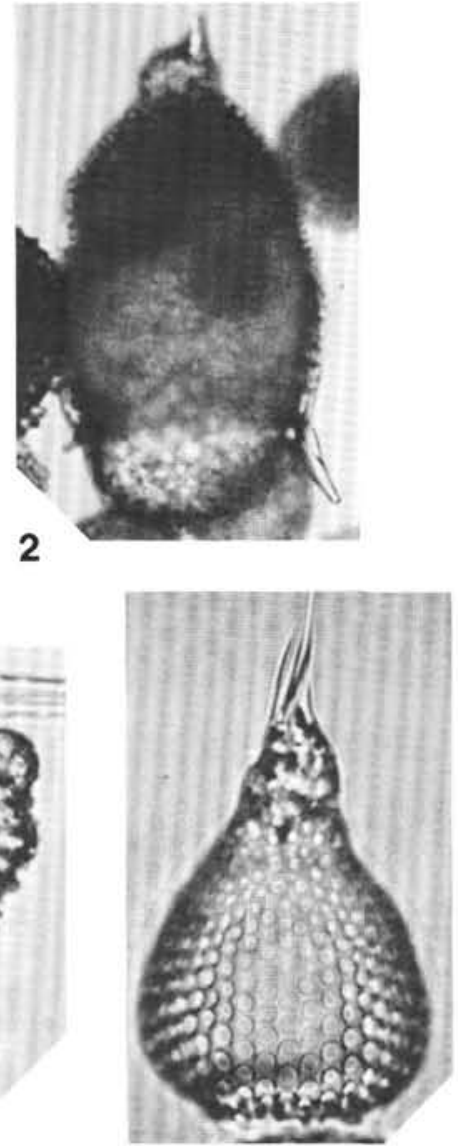

4

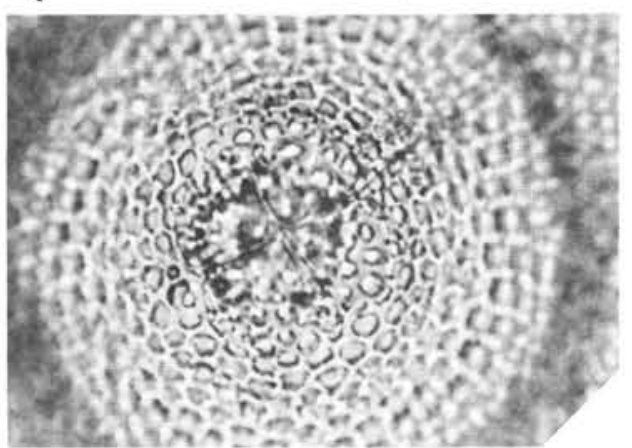

10

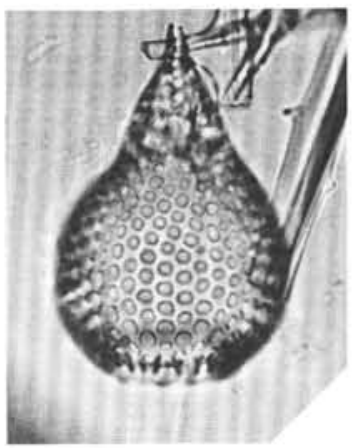

3

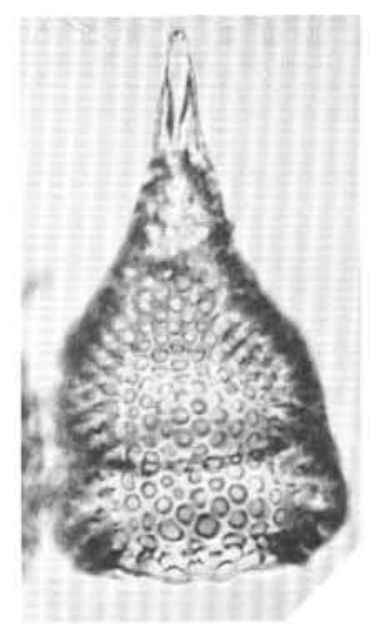

7

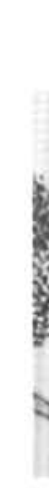



5

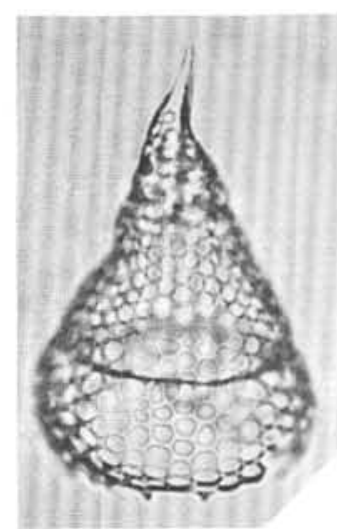

6

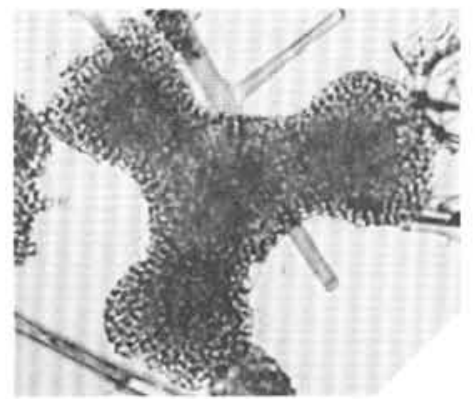

11

Plate 3. 1, 2. Thyrsocyrtis clausa, $\times 143$, Sample 120-751A-14H-CC. 3, 4. Anthocyrtidium sp., $\times 293$; (3) Sample 120-752A-14H-CC, (4) Sample 120-747A-8H-6, 45-47 cm. 5, 6. Lamprocyrtis(?) cf. hannai, $\times 293$; (5) Sample 120-751A-13H-CC, (6) Sample 120-748B-6H-1, $65-67 \mathrm{~cm}$. 7. Lamprocyclas sp., $\times 293$, Sample 120-748B-8H-CC. 8. Velicucullus altus, $\times 293$, Sample 120-748B-8H-2, 45-47 cm. 9, 10. Velicucullus cf. oddgurneri; (9) $\times 143$, Sample 120-748B-8H-6, $45-47 \mathrm{~cm},(10) \times 293$, Sample $120-748 \mathrm{~B}-8 \mathrm{H}-6,45-47 \mathrm{~cm}$. 11. Rhopalodictyum sp., $\times 143$, Sample 120-747A-6H-CC. 




1

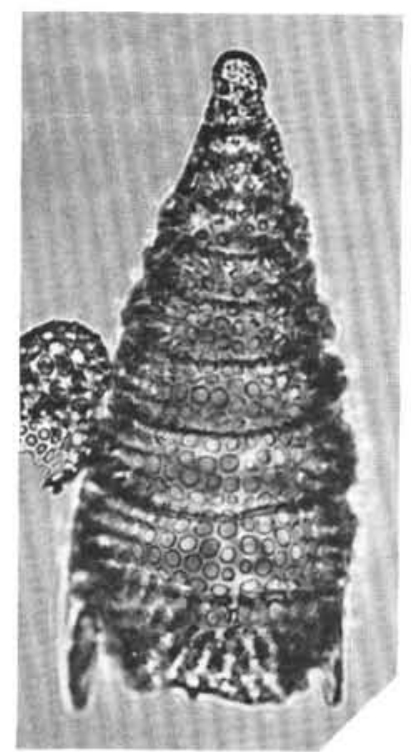

5

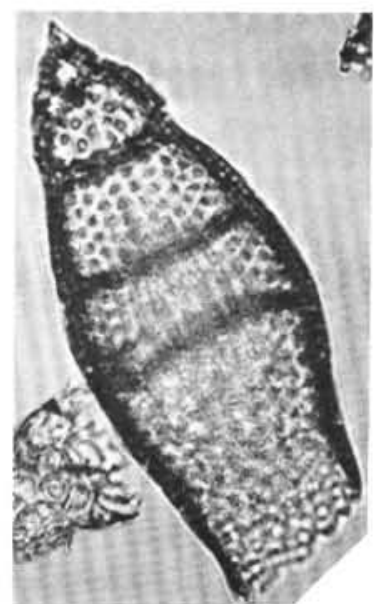

2

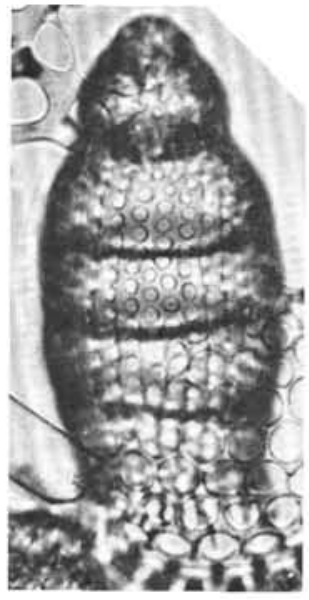

6

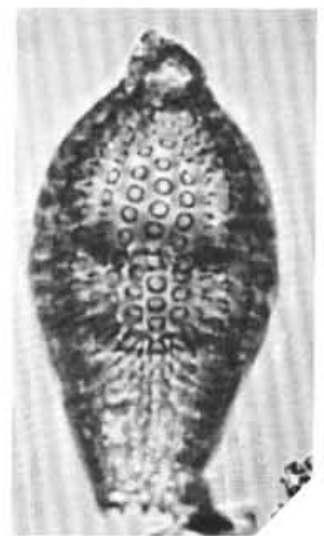

3

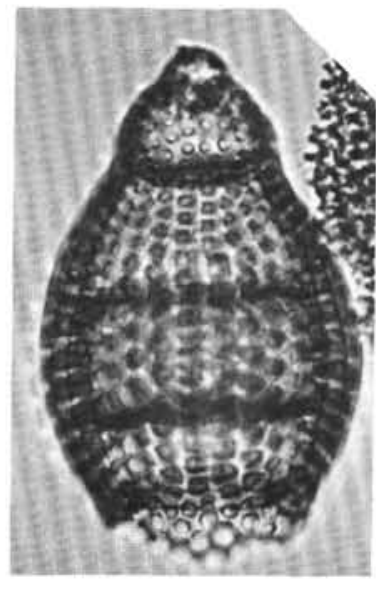

7

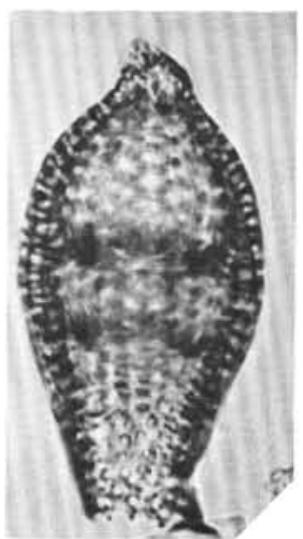

4

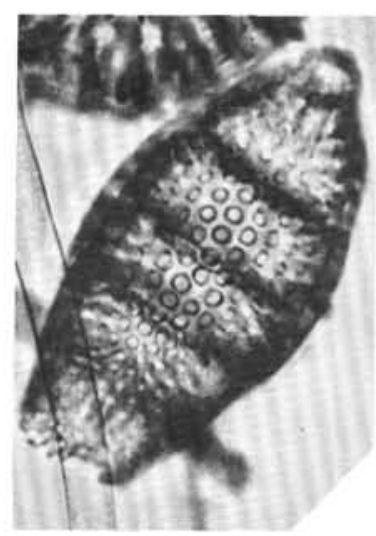

8

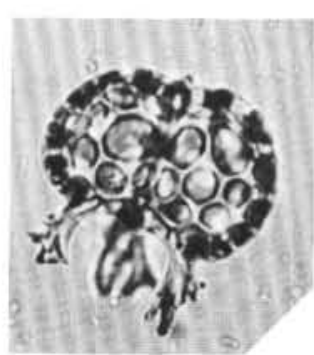

9

Plate 4. 1, 2. Eucyrtidium cienkowskii, $\times 293$, Sample $120-751 \mathrm{~A}-16 \mathrm{H}-6,98-100 \mathrm{~cm} .3,4$. Eucyrtidium punctatum, $\times 231$, Sample 120-748B-6H-1, 65-67 cm. 5. Stichophormis sp., $\times 231$, Sample 120-751A-16H-5, 98-100 cm. 6. Eucyrtidium calvertense, $\times 293$, Sample 120-747A-8H-CC. 7. Eucyrtidium sp. A, $\times 293$, Sample 120-751A-13H-CC. 8. Eucyrtidium sp. B, $\times 293$, Sample 120-747A-10H-CC. 9. Corythospyris fiscella, $\times 293$, Sample 120-748B-8H-6, 45-47 cm. 


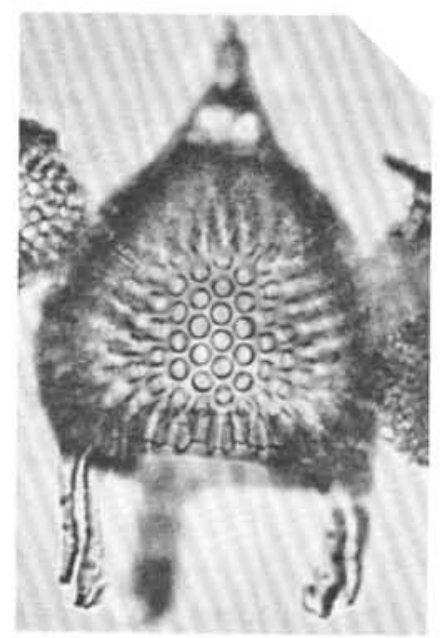

1

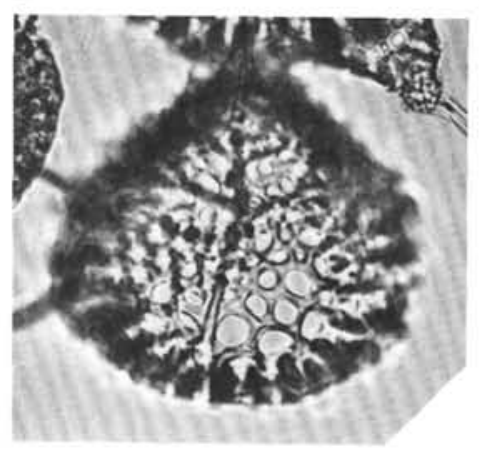

5

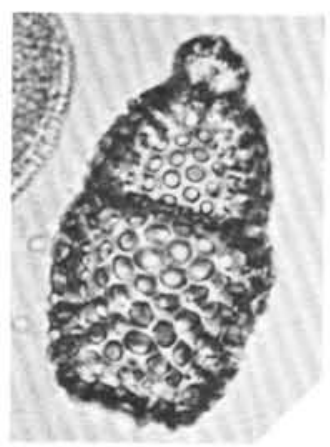

7

8

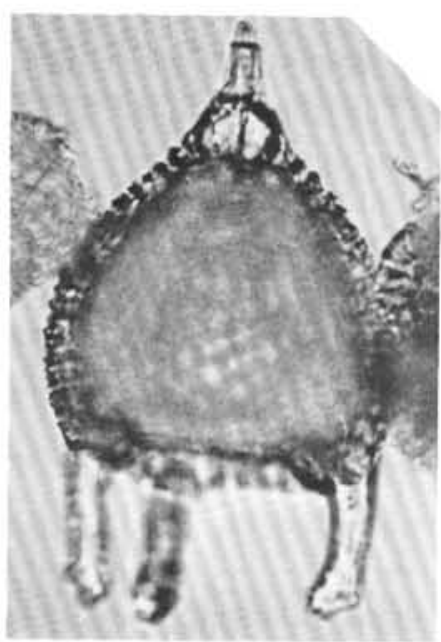

2

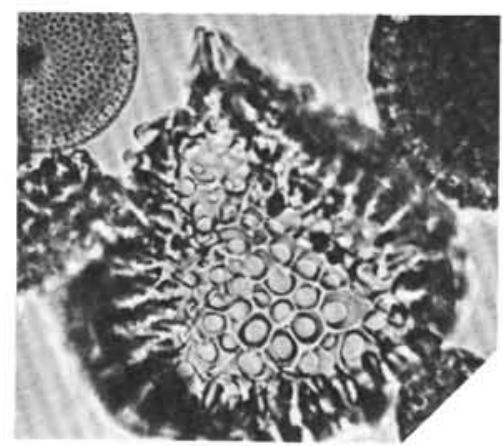

6

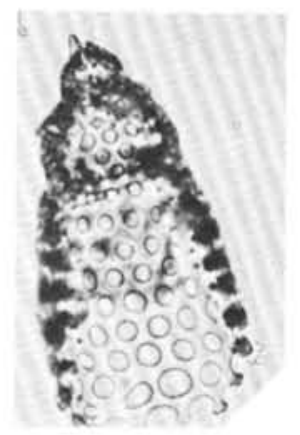

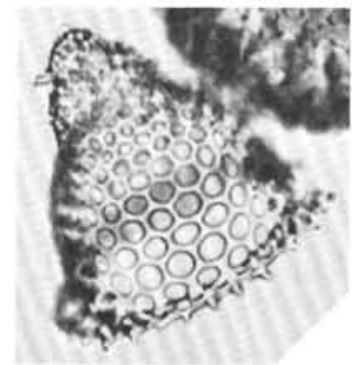

9

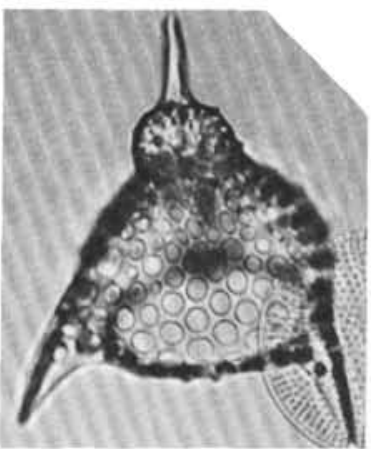

3

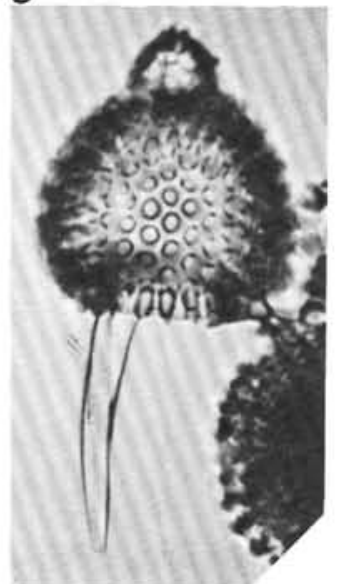

4

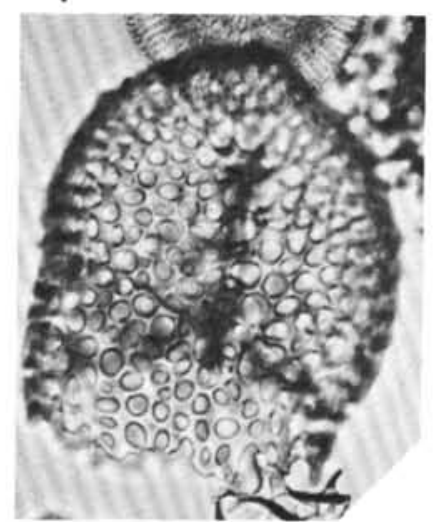

10

Plate 5. 1, 2. Lychnocanoma sp. C, $\times 231$, Sample 120-748B-6H-7, $45-47 \mathrm{~cm} .3$. Pterocanium sp., $\times 293$, Sample 120-747A-8H-CC. 4. Lychnocanoma sp. B, $\times 231$, Sample 120-751A-12H-CC. 5, 6. Spongomelissa dilli, $\times 231$, Sample 120-751A-12H-CC. 7. Cyrtocapsella robusta, $\times 293$, Sample 120-748B-8H-6, $45-47 \mathrm{~cm}$. 8. Calocyclas $\mathrm{cf}$. semipolita, $\times 293$, Sample 120-748B-8H-6, $45-47 \mathrm{~cm} .9$. Cycladophora sp., $\times 293$, Sample 120-748B-8H-5, 45-47 cm. 10. Dendrospyris megalocephalis, $\times 293$, Sample 120-751A-12H-CC. 